\title{
EFFECT OF THE HORIZONTAL COMPONENT OF EARTHQUAKE ON THE BUCKLING OF CONCRETE SPHERICAL SHELLS
}

by

Nathalie Elena Moreno Madueño

B. ENG. University of Zulia, Maracaibo, Venezuela, 2016

\author{
A thesis \\ presented to Ryerson University \\ in partial fulfillment of the \\ requirement for the degree of \\ Master of Applied Science \\ in the program of \\ Civil Engineering
}

Toronto, Ontario, Canada, 2019

(C) Nathalie Elena Moreno Madueño, 2019 


\begin{abstract}
AUTHOR'S DECLARATION
I hereby declare that I am the sole author of this thesis. This is a true copy of the thesis, including any required final revisions, as accepted by my examiners.

I authorise Ryerson University to lend this thesis to other institutions or individuals for the purpose of scholarly research.

I further authorize Ryerson University to reproduce this thesis by photocopying or by other means, in total or in part, at the request of other institutions or individuals for the purpose of scholarly research.
\end{abstract}

I understand that my thesis may be made electronically available to the public. 


\title{
EFFECT OF THE HORIZONTAL COMPONENT OF EARTHQUAKE ON THE BUCKLING OF CONCRETE SPHERICAL SHELLS
}

\author{
Nathalie Elena Moreno Madueño \\ Master of Applied Science, 2019 \\ Department of Civil Engineering \\ Ryerson University
}

\begin{abstract}
The buckling failure of reinforced concrete spherical shell structures under the effect of the horizontal component of earthquake is investigated using a finite element method over a wide range of shell configurations. For this effect, two different loading case scenarios are considered; first, the shell is analyzed under the effects of the vertical seismic component alone. Then, the model is reanalyzed under the same loading conditions plus the horizontal earthquake component, taking into account two different horizontal-to-vertical earthquake spectral ratios. It is concluded that including the horizontal component of earthquake can result in a reduction in the buckling capacity of this type of structure; the impact of which is highly influenced by the horizontal-tovertical earthquake spectral ratio and the shell geometry. It is also observed that the formulation adopted by ACI slightly overestimates the buckling capacity of spherical shells especially when horizontal seismic effects are included.
\end{abstract}




\section{ACKNOWLEDGEMENTS}

Firstly, I would like to thank God almighty for His great love and grace. I especially want to thank Him this time for providing all the means to accomplish this thesis, for giving me the courage, direction and strength I needed in difficult times; and for always providing through different people the support I needed. To Him be the glory and Honour.

I would also like to express my sincere gratitude to my supervisor Dr. Kianoush, in the first place, for believing in me and giving me the opportunity to carry out my master's studies, but also for his patience, encouragement, support and instruction at all times; the doors of his office were always open whenever I had to discuss any doubt or problem.

I would also like to thank Dr. Moslemi who always steered me in the right direction; for always taking the time to help me, for the extended discussions, for giving an answer to my many questions, and for providing valuable suggestions that greatly contributed to the improvement of this thesis.

Finally, I am must express my very profound gratitude to my family; that even in the distance, their warm love and guidance have always been with me; especially my mom for providing unfailing support and encouragement throughout my whole life.

This accomplishment would not have been possible without your help. Thank you all. 


\section{TABLE OF CONTENTS}

AUTHOR'S DECLARATION

ABSTRACT

ACKNOWLEDGEMENTS

LIST OF TABLES viii

LIST OF FIGURES $\quad$ x

LIST OF SYMBOLS X xiii

1. INTRODUCTION 1

1.1 Background 1

1.2 Objectives and scope 4

$\begin{array}{lll}1.3 & \text { Research significance } & 6\end{array}$

1.4 Thesis layout 7

2. LITERATURE REVIEW 9

2.1 Introduction 9

2.2 Classical shell theory 9

2.3 Governing equations of general linear shell theory 13

2.4 Definition of spherical shells 20

2.5 Theory of elastic stability 22

2.6 History of buckling theories of thin spherical shells 26

2.7 ACI 372R-13 design method 36

3. FINITE ELEMENT MODELING 40

3.1 Introduction 40

3.2 Finite element method 41

3.3 Element selection 43

3.4 Mesh size 45

$\begin{array}{lll}3.5 & \text { Summary } & 52\end{array}$ 
4. BUCKLING OF SPHERICAL SHELLS 53

4.1 Introduction 53

4.2 Models properties 54

4.3 Finite element modelling of reinforced concrete shells 56

4.4 Linear analysis $\quad 59$

4.4.1 Linear eigenvalue buckling analysis $\quad 60$

4.4.2 Comparison with classical buckling equation 63

$\begin{array}{lll}4.5 & \text { Nonlinear analysis } & 65\end{array}$

4.5.1 Imperfection 66

4.5.2 Static Riks Analysis $\quad 70$

4.5.3 Comparison of results with the ACI formulation 75

4.5.4 Comparison between the bifurcation and snap-through buckling response 77

4.5.5 Effect of base fixity 79

$\begin{array}{lll}4.6 & \text { Summary } & 81\end{array}$

5. BUCKING RESPONSE OF SPHERICAL SHELLS SUBJECTED TO EARTHQUAKE LOADING $\quad 84$

$\begin{array}{lll}5.1 & \text { Introduction } & 84\end{array}$

5.2 Natural frequency extraction 85

$\begin{array}{lll}5.3 & \text { Horizontal component of earthquake } & 87\end{array}$

5.4 Vertical component of earthquake 91

5.5 Finite element modeling 92

5.6 Finite element results 95

5.7 Comparison of FE results with current practice 99

5.8 Summary 103

6. SUMMARY, CONCLUSIONS AND RECOMMENDATIONS 106

6.1 Summary 106

$\begin{array}{lll}6.2 \text { Conclusions } & 108\end{array}$ 
6.3 Recommendations for future studies 110 APPENDIX A: COMPARISON BETWEEN FIXED AND HINGED BOUNDARY

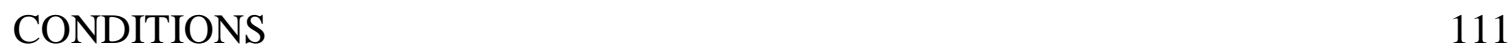

APPENDIX B: INPUT FILE OF MODEL 2 118

$\begin{array}{ll}\text { REFERENCES } & 124\end{array}$ 


\section{LIST OF TABLES}

Table 1. Load combinations for design of spherical shells as per ACI372-13

Table 2. Mesh size selection for each model

Table 3. Geometry of the models

Table 4. Material properties

Table 5. Reinforcement arrangement of the models

Table 6. Summary of FE buckling analysis results (linear theory)

Table 7. Comparison of linear buckling pressure (FE vs. classical buckling equation)

Table 8. Effect of different levels of geometric imperfections

Table 9. Geometric imperfection parameters

Table 10. Analytical linear and nonlinear buckling pressure

Table 11. Nonlinear buckling capacity (FE)

Table 12. Nonlinear buckling response (ACI vs. FE)

Table 13. Bifurcation vs. Snap-through buckling (FE)

Table 14. Effect of base fixity on buckling capacity (FE)

Table 15. Free vibration analysis results

Table 16. Summary of response spectrum parameters

Table 17. Spectral accelerations corresponding to the horizontal earthquake component 
Table 19. Seismic loading data for FE analysis (horizontal and vertical excitation)

Table 20. Buckling pressure from FEA under seismic action (ACI372R-13)

Table 21. Buckling pressure from FEA under seismic action (ASCE7-10)

Table 24. ACI vs. FE results under combined seismic action (seismic loading as per ACI372R-13)

Table 25. ACI vs. FE results under combined seismic action (seismic loading as per ASCE7-10) 


\section{LIST OF FIGURES}

Figure 1. Stresses in a shell differential element (Adapted from Billington, 1965) .................. 11

Figure 2. Differential Shell element (Adapted from Billington, 1965) ................................... 14

Figure 3. Stress resultants (Adapted from Billington, 1965) ................................................ 14

Figure 4. Shell element after deformation (Adapted from Billington, 1965) .......................... 17

Figure 5. Surface of revolution (Adapted from Farnsworth Jr., 1998) ..................................... 21

Figure 6. Dome vs arch (Adapted from Farnsworth Jr., 1998) ............................................. 22

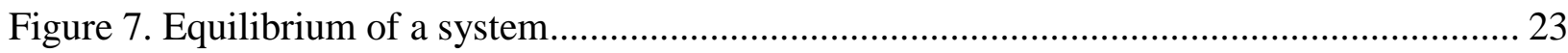

Figure 8. Buckling failure of shell structures (Adapted from Ventsel et al., 2001).................. 24

Figure 9. Buckling failure of a dome structure (Adapted from Hamed, 2010) ........................ 25

Figure 10. Geometry of spherical shell (Adapted from Timoshenko and Gere, 1961) .............. 26

Figure 11. Summary of sphere-test performance (Adapted from Carlson et al., 1967) .............. 28

Figure 12. Comparison between different boundary conditions (Adapted from Huang, 1963) ... 30

Figure 13. Load-deflection curves and bifurcation buckling of spherical caps with various values of the shallowness parameter (Adapted from Bushnell, 1981) ........................................ 32

Figure 14. Effect of initial imperfections for $\boldsymbol{v}=0.3$ (Adapted from Krenzke and Kiernan, 1965)

Figure 15. Comparison of actual and FEA predicted failure of spherical shell. 42 
Figure 16. ABAQUS element families 44

Figure 17. Finite element geometry; (a) Node ordering, (b) Number of integration points ......... 45

Figure 18. Inaccuracy of FE solution as a function of mesh size ......................................... 46

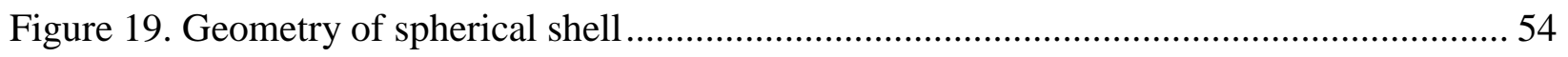

Figure 20. Spherical shell reinforcement (Adapted from https://www.styrouae.com)............... 56

Figure 21. Shell reinforcement (ABAQUS keyword modification) ...................................... 57

Figure 22. Radial rebars in axisymmetric shell elements (Adapted from ABAQUS manual) ..... 58

Figure 23. Circumferential rebars in axisymmetric shell elements (Adapted from ABAQUS

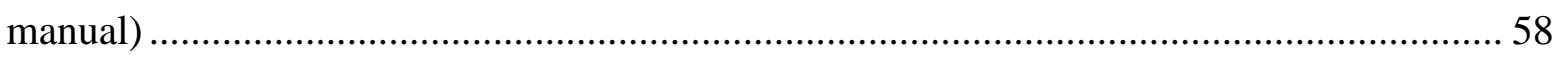

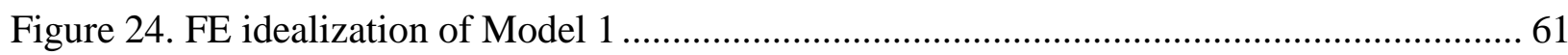

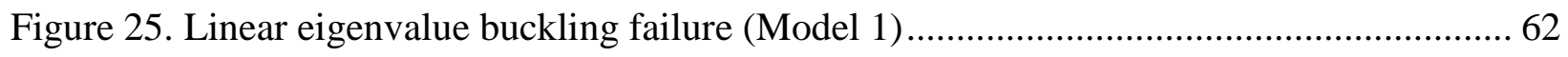

Figure 26. Comparison of linear buckling pressure (FE vs. classical buckling equation)........... 64

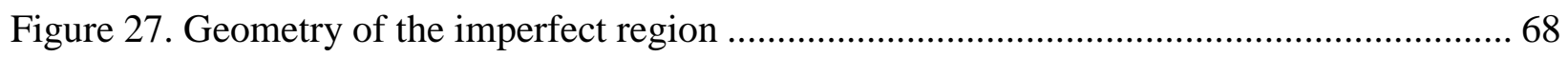

Figure 28. Results obtained from FE Static Riks analysis ............................................... 72

Figure 29. Snap-through buckling failure of Model 1 ................................................... 75

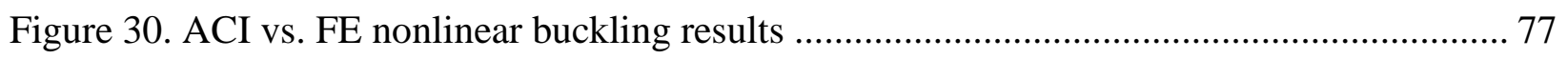

Figure 31. Bifurcation vs. Snap-through buckling (FE) ............................................... 78

Figure 32. Comparison between ACI and FE for different types of boundary conditions ........... 81 
Figure 33. Design response spectrum (Adapted from ASCE7-10).

Figure 34. Response spectrum used in analysis for horizontal component of earthquake 90

Figure 35. Self-weight loading of the dome 94

Figure 36. Buckling pressure from FEA under seismic action (ACI372R-13) ........................... 96

Figure 37. Buckling pressure from FEA under seismic action (ASCE7-10)_.............................. 97

Figure 38. ACI vs. FE results under pure vertical seismic action................................................ 99

Figure 39. ACI vs. FE results under combined seismic action (seismic loading as per ACI372R13) 102

Figure 40. ACI vs. FE results under combined seismic action (seismic loading as per ASCE7-10) 103 


\section{LIST OF SYMBOLS}

$a \quad$ radius of the sphere, $\mathrm{m}$ - Timoshenko and Gere's notation

D dead loads or related internal moments and forces

E modulus of elasticity, $\mathrm{MPa}$

Ec modulus of elasticity of concrete under short-term load, psi (MPa) - ACI's notation

$E_{c} \quad$ concrete modulus of elasticity, $\mathrm{MPa}$

$E_{c o} \quad$ composite modulus of elasticity, $\mathrm{MPa}$

$E_{s} \quad$ steel modulus of elasticity, $\mathrm{MPa}$

Ev vertical seismic load, $\mathrm{lb} / \mathrm{ft}^{2}(\mathrm{kPa})$

Ev vertical seismic load, $\mathrm{lb} / \mathrm{ft}^{2}(\mathrm{kPa})-\mathrm{ACI}$ 's notation

$h \quad$ thickness of the shell, $\mathrm{m}$ - Timoshenko and Gere's notation

hd dome shell thickness, in (mm) - ACI's notation

L uniformly distributed dome live load, $\mathrm{lb} / \mathrm{ft}^{2}(\mathrm{kPa})$

LL live Load

LPF Load Proportionality Factor

$N_{n}, Q_{n}, M_{n} \quad$ Resultant forces in the middle surface of the shell - In the governing equation of shells

$P u \quad$ factored design load on dome shell, $\mathrm{lb} / \mathrm{ft}^{2}(\mathrm{kPa})-\mathrm{ACI}$ 's notation 
$q \quad$ uniform external pressure

$q_{c r} \quad$ critical external pressure

$R \quad$ main radius of the sphere, $\mathrm{m}$

rd mean radius of dome, $\mathrm{ft}(\mathrm{mm})$ - ACI's notation

Rimp average radius of curvature measured over a region of diameter

S uniformly distributed snow load in accordance with ASCE 7, $\mathrm{lb} / \mathrm{ft}^{2}(\mathrm{kPa})$

$\mathrm{S}_{\mathrm{D} 1}$ the design spectral response acceleration parameter at 1-s period

$\mathrm{S}_{\mathrm{DS}}$ the design spectral response acceleration parameter at short periods

$\mathrm{T}$ the fundamental period of the structure, $\mathrm{s}$

$t \quad$ thickness of the shell, $\mathrm{m}$

$\mathrm{T}_{0} \quad \mathrm{~T}_{0}=0.2 \frac{\mathrm{S}_{D 1}}{\mathrm{~S}_{D S}}(\mathrm{~s})$

$\mathrm{T}_{\mathrm{L}} \quad$ Long-period transition period (s)

$\mathrm{T}_{\mathrm{S}} \quad \mathrm{T}_{S}=\frac{\mathrm{S}_{D 1}}{\mathrm{~S}_{D S}}(\mathrm{~s})$

$\phi \quad$ strength-reduction factor

$\sigma \quad$ stress

$\beta c \quad$ factor of buckling strength due to creep, nonlinearity and cracking of concrete

$\sigma_{c r} \quad$ critical stress 
$\beta i \quad$ buckling reduction factor for geometrical imperfection - ACI's notation

Bimp factor of buckling strength due to imperfection

$\varepsilon_{n} \quad$ normal strains in the $\mathrm{n}$ direction - In the governing equation of shells

$\gamma_{\mathrm{n}} \quad$ shear strains - In the governing equation of shells

$\sigma_{n} \quad$ normal stresses in the n direction - In the governing equation of shells

$\tau_{\mathrm{n}} \quad$ shear stresses - In the governing equation of shells

$v \quad$ poisson's ratio.

$\mathrm{z} \quad$ thickness - In the governing equation of shells

$\lambda \quad$ shallowness parameter $=\left[12\left(1-v^{2}\right)\right]^{1 / 4}(R / h)^{1 / 2} \theta$

$\lambda \quad$ the eigenvector or mode of vibration - free vibration analysis

$\rho \quad$ non-prestressed reinforcement area-to-concrete area

$\theta \quad$ half-subtended angle of spherical cap

$\omega \quad$ frequency

$[\mathrm{K}] \quad$ stiffness matrix

[M] mass matrix 


\section{CHAPTER 1}

\section{INTRODUCTION}

\subsection{Background}

Spherical shells, commonly known as domes, have been used around the world for more than two thousand years. From the Middle East to the Roman Empire, passing through many other cultures, these structures were used as roofs of their most important buildings, making them an iconic symbol of the ancient world.

A proof of this is provided by "the Pantheon", one of the oldest existing structures which dates back to $126 \mathrm{AD}$. This marvelous structure count with a diameter of 43 meters and a rise of 22 meters above its base, supported by a series of arches that rested at the same time on eight piers; the majesty and engineering of this building served as inspiration for the development of future dome structures. One other good example of the widespread use of these structures is the "Dome of the Rock" located in Jerusalem, this one originally dates from 691AD; however, due to the impact of three earthquakes in 808AD, 846AD and 1015AD; the structure finally collapsed during the last one, being later rebuilt in 1027-1028. The history of this particular dome shows how durable these structures could be; however, even if they can provide great resistance; earthquakes always represent an outstanding hazard that needs to be considered.

As noted, in the beginning the use of this type of structure was mainly oriented to religious purposes; as they were always associated with some kind of spiritual symbolism. Nonetheless, later in history, its use was extended to other purposes, since it was demonstrated that they not only offer mighty architectural configurations, but they were also highly resistant structures, capable of providing long spans with no intermediate elements, and a minimum of material.

The resistance of these structures has its origin in their geometry; since, thanks to 
their configuration, the internal forces of compression and tension are organized in such a way that they allow the structure to withstand large loads with an optimum performance. However, like any structure, they do not have an infinite resistance, and once again it is their geometry, more specifically their thickness, that is the source of their failure. This complexity in their behavior has led numerous scientists and engineers to perform in-depth analyses in this area.

Even though these structures have been used for so many years, it was not until during the last two centuries that formal investigations about their behavior have been developed and documented, leading to formulations that provide a very close solution to the problem.

As may be seen, it has been a work of almost 200 years that has driven to precise formulations; however, at the beginning, as in any unknown area, the problem was not addressed in the correct angle; since the main criteria at the time of designing were based on the resistance of membrane stresses. Nevertheless, later, the results obtained from experimental investigations showed that this was not the right approach to analyze these elements, since the shells were failing under much lower stresses. But, it was not until the development of the theory of instability, when it was discovered that the failure of these structures was not related to a material failure, but to an instability problem, which is closely associated with the stiffness of the structure.

This type of failure that the structures were experiencing according to the theory of instability, received the name of buckling. Timoshenko (1936), the main author of this theory, developed along with it, a series of formulations that predicted the buckling failure of different structures, including that of spherical shells, which is commonly known as the "classical buckling pressure".

However, later on, this formulation faced significant criticisms due to the many simplifying assumptions made in the approach and significant discrepancies with experimental results; leading later to the development of new formulations proposed by the 
same author, and also by many other researchers (Karman and Tsien (1939), Krenzke and Kiernan (1965), Bushnell (1981), Zarghamee and Heger (1983), among many others)

Since then, a large number of investigations have been carried out in order to redefine the proposed formulation; one of these many attempts is the formulation proposed by Zarghamee and Heger (1983). Based on the results of previous investigations along with a great deal of analytical work of their own, they finally proposed a formulation that could predict the critical buckling pressure of a spherical cap in good agreement with experimental results.

As the results obtained were found quite accurate, the American Concrete Institute (ACI) decided to adopt the same formulation in its ACI-372 (2013) code for the design of the roof of circular prestressed concrete structures. However, in the estimation of the critical buckling pressure only the effects of dead load, live load, and vertical component of earthquake were accounted for and the effect of the horizontal component of earthquake was totally neglected. On one hand, this could be taken as an appropriate and logical decision; since, after studying the behavior of this type of structure, it is observed that the main type of load influencing their behavior is the one perpendicular to the surface; which in this case, comes from gravitational loads. However, on the other hand, it is known that when an earthquake strikes a structure, the horizontal component shows large magnitudes, usually becoming the main perturbation affecting the behavior of the entire structure.

Therefore, as mentioned before, a more detailed analysis of these structures under the effect of seismic action seems essential; as an overestimation of the structural capacity could be catastrophic from the design perspective. However, among the many different investigations performed in the area, little or no information is provided about this particular issue. For this reason, it is necessary to provide a quantitative answer to this problem in an effort to verify the assumptions made by ACI and/or provide results that could lead to some improvements in the proposed formulations. 
In this investigation, the finite element technique is used to predict the response of spherical shells under the influence of the horizontal component of earthquake; in order to provide some useful information for the development of design guide for dome structures, and improvement of the current code.

\subsection{Objectives and scope}

As previously mentioned, there is little information available about the buckling response of spherical shells under the action of seismic loading; therefore, the general purpose is to contribute and produce new information that can provide a better understanding of such an effect.

In the same vein, driven by the lack of information and the assumption of ACI to neglect the effect of the horizontal excitation of earthquake; the main objective of this study is then to evaluate the response of these structures under the effect of the horizontal component of earthquake and determine the accuracy of the design procedure proposed by ACI.

In order to achieve this main objective, additional steps are also necessary throughout the process. Prior to the analysis, a proper understanding of the problem is needed along with a deep comprehension of all the theories that have been developed to evaluate this type of failure. Once the basis for ensuring a correct analysis has been established, then a finite element technique must be developed to ensure an adequate idealization of the problem and consequently accurate results.

In summary, the main objectives of this research are as follows:

1) Propose a rigorous finite element (FE) technique capable of accurately predicting the buckling failure of spherical shells.

2) Verify the proposed FE technique by comparing the numerical results with those 
obtained from the analytical solution under both linear and nonlinear buckling failure conditions.

3) Understand the effect of a wide range of parameters on the buckling response of spherical shells, such as: radius-to-thickness ratio, span-to-rise ratio, boundary conditions, geometric imperfections and rebar arrangement in member's crosssection.

4) Examine the effect of the inclusion of the horizontal component of earthquake on the buckling behavior of spherical shells with different geometric configurations.

5) Investigate the validity of ACI approach in estimating the buckling capacity of concrete spherical shells for design or evaluation purposes by comparing the results from FE method with those recommended by the code.

On the other hand, the scope of this study is summarized as follows:

1) The spherical shells are assumed to have either hinged or fixed boundary condition at their base. The effect of other possible base fixities is not studied here.

2) All the loads, including seismic are applied as equivalent static load on the structure.

3) The material used is reinforced concrete, defined in accordance with ACI specifications.

4) In the FE modeling of the structure, all the materials are assumed as linear static. Yielding of rebar, cracking/crushing of concrete, and creep effects are not accounted for. 


\subsection{Research significance}

In this investigation, a FE method is developed to analyze the buckling behavior of spherical shell structures. The proposed model is verified by observing a strong correlation with analytical results available in the literature. The resulting numerical model can be used to analyze not only simple cases but also complex shell problems with a high degree of precision.

As mentioned before, the buckling failure analysis of concrete spherical shells with different geometric configurations, boundary conditions and under different load cases is made possible; as a result of which important conclusions about the buckling behavior of these structures can be obtained.

One of the parameters that has never been addressed in previous investigations is the effect of having multiple instead of a single layer of reinforcement on the buckling behavior of spherical shells; probably because it has not been expected to have considerable effect. However, since there is little information available regarding this aspect, it is relevant to address and provide conclusions to it.

The same situation is noticed regarding the effect of base boundary condition in spherical shells with imperfections. A lot is known about the role having different base fixities can play in buckling failure of a perfect dome; however, when an imperfection is included in the geometry, there is not a conclusive theory with respect to the edge conditions. Therefore, this investigation intends to provide more information in regards to this particular problem and perhaps a response for future studies.

At the same time, for each case study, both linear and nonlinear analyses are performed using the developed FE technique, and as previously mentioned, the results are compared with the current analytical formulations. This not only seeks to verify the technique used, but also to support the assumptions and conclusions made by the analytical 
formulations related to each case.

However, the most important aspect studied in this research is the effect of inclusion of the horizontal component of earthquake on the buckling behavior of spherical shells. Given the fact that there is little information on this matter and that the impact this could have on design is unknown, it is necessary to provide more data on this point.

Additionally, the common practices and current codes, such as ACI 372-13, neglect the effect of horizontal component of earthquake in the design method of spherical shell structures. Since this assumption does not have any rationale, it is necessary to provide further clarifications, in order to verify the accuracy of the current approach to ensure an accurate and a safe design procedures for these types of structures.

\subsection{Thesis layout}

This thesis is divided into six chapters, in accordance with the objectives and scope previously explained. The first chapter presents and gives a general introduction to the problem, specifying at the same time the objectives and scope of the investigation as well as a brief explanation of the importance of this research, ending with a description of its outline.

The second chapter, presents a summary of the most important investigations that have been used for the development of this thesis, as well as an explanation of the most relevant theories that have also been essential for the understanding of the problem, such as the theory of thin shells, theory of elastic stability and the classical buckling theory. Additionally, a summary of the design method proposed by ACI is also described in this chapter.

Subsequently, Chapter 3 discusses all the aspects of the idealization of the problem, from a description of the Finite Element Method to the definition of the mesh size for each one of the models, describing as well, the software and elements used throughout the investigation. 
Chapter 4, initially presents the properties of the models, describing also the characteristics and conditions under which each case is analyzed. This is followed by an explanation of the finite element technique used in this specific chapter for the analysis of the linear and nonlinear buckling failure of the spherical shells, comparing each case with the classical buckling equation and ACI formulation, respectively.

In Chapter 5, a detailed nonlinear finite element analysis is carried out; starting with a frequency extraction analysis in order to determine the free vibration dynamics of the models. After defining the earthquake excitations to be used, finite element analysis is performed to determine the buckling failure response under two different loading conditions: 1) Pure vertical and 2) Combined vertical and horizontal seismic action. Once the results are obtained, a comparative study is carried out between them and that of ACI approximation.

To conclude, in Chapter 6, a summary of the entire investigation is presented, followed by a description of the main conclusions obtained from this study, and lastly some recommendations for future studies are proposed as well. 


\section{CHAPTER 2}

\section{LITERATURE REVIEW}

\subsection{Introduction}

In this chapter, a summary of the previously developed theories and investigations related to this research is presented.

During the first part of this chapter and before delving into the problem, it is necessary to provide a description of the general behavior of shell elements. Once the basis has been established, a summary of the theories proposed to predict the behavior is presented, such as the theory of thin shells and the theory of instability.

Subsequently, the problem is focused on the specific case of spherical shells; providing first a definition of the behavior of these elements, followed by an explanation of the evolution of their design. Additionally, the improvements and discoveries that have been developed over the years to provide a good correlation between the analytical, numerical and experimental results are also described throughout this chapter.

Finally, the requirements and specifications provided by ACI for the design of these structures are introduced in the last part of this chapter, together with the assumptions made for this formulation.

\subsection{Classical shell theory}

Shells are three-dimensional elements characterized by having one dimension much smaller than the other two. The main characteristic of this type of element is that it is able to sustain applied loads by developing membrane in-plane stresses and bending moments at the same time. However, the formulations to accurately represent this behavior have always been a complex problem in the mechanics of materials that numerous researchers have attempted to study for many years. 
One of the methods that the researchers have found useful to study these complex elements is by reducing the general problem to simpler particular cases. In this respect, the general shell problem is divided according to their thickness, into two categories of shell elements: thick shells and thin shells.

However, when the formulations for these elements were being developed, in the beginning, the dividing line between them was not precisely established and therefore, thin elements were just defined as elements with a thickness-to-length ratio much smaller than the unity $(\mathrm{z} / \mathrm{r}<<1)$. This assumption not only represented the divisive parameter, but also the most important postulate of the theories.

The first theory of thin shells was proposed by Love (1888), based on the principles proposed some years earlier by Poisson (1827) and Kirchhoff (1859) concerning shells and elasticity. The main assumption of this theory, as previously stated, is that the thickness is much smaller in comparison to other dimensions; as a result of which the higher powers of $\mathrm{z} / \mathrm{r}$ are immediately neglected, and therefore, the formulation is transformed from a threedimensional to a two-dimensional problem that focuses on analyzing the middle surface of the shell.

The second postulate assumes small strains and displacements, and together with Hooke's law (Hooke, 1678) neglects the higher order terms in the formulation, resulting then in a system described by a set of geometrical linear equations, as follows:

$$
\begin{gathered}
\varepsilon_{\mathrm{x}}=\frac{\sigma_{\mathrm{x}}}{E_{\mathrm{x}}}-\frac{v_{\mathrm{xy}}}{E_{\mathrm{y}}} \sigma_{\mathrm{y}}-\frac{v_{\mathrm{xz}}}{E_{\mathrm{z}}} \sigma_{\mathrm{z}}, \\
\varepsilon_{\mathrm{y}}=\frac{\sigma_{\mathrm{y}}}{E_{\mathrm{y}}}-\frac{v_{\mathrm{yx}}}{E_{\mathrm{x}}} \sigma_{\mathrm{x}}-\frac{v_{\mathrm{yz}}}{E_{\mathrm{z}}} \sigma_{\mathrm{z}}
\end{gathered}
$$




$$
\begin{gathered}
\varepsilon_{\mathrm{z}}=\frac{\sigma_{\mathrm{z}}}{E_{\mathrm{z}}}-\frac{v_{\mathrm{zx}}}{E_{\mathrm{z}}} \sigma_{\mathrm{x}}-\frac{v_{\mathrm{zy}}}{E_{\mathrm{z}}} \sigma_{\mathrm{y}} \\
\gamma_{\mathrm{xy}}=\frac{\tau_{\mathrm{xy}}}{G_{\mathrm{xy}}}, \quad \gamma_{\mathrm{xz}}=\frac{\tau_{\mathrm{xz}}}{G_{\mathrm{xz}}}, \quad \gamma_{\mathrm{yz}}=\frac{\tau_{\mathrm{yz}}}{G_{\mathrm{yz}}},
\end{gathered}
$$

According to Figure 1 and the coordinate system described by Billington (1965), $\varepsilon_{x}$, $\varepsilon_{y}$ and $\varepsilon_{z}$ represent the normal strains in $\mathrm{X}, \mathrm{Y}$ and $\mathrm{Z}$ direction; $\sigma_{\mathrm{x}}, \sigma_{\mathrm{y}}$ and $\sigma_{\mathrm{z}}$ are the respective stresses; $E_{x}, E_{y}$ and $E_{z}$ describe the tendency of the element to deform along the corresponding axis known as modulus of elasticity; $G_{x y}, G_{x y}$ and $G_{y z}$ represent the shear modulus or modulus of rigidity, while $\gamma_{\mathrm{xy}}, \gamma_{\mathrm{xz}}, \gamma_{\mathrm{yz}}$ and $\tau_{\mathrm{xz}}, \tau_{\mathrm{yz}}, \tau_{\mathrm{xy}}$ are the corresponding shear strains and stresses.

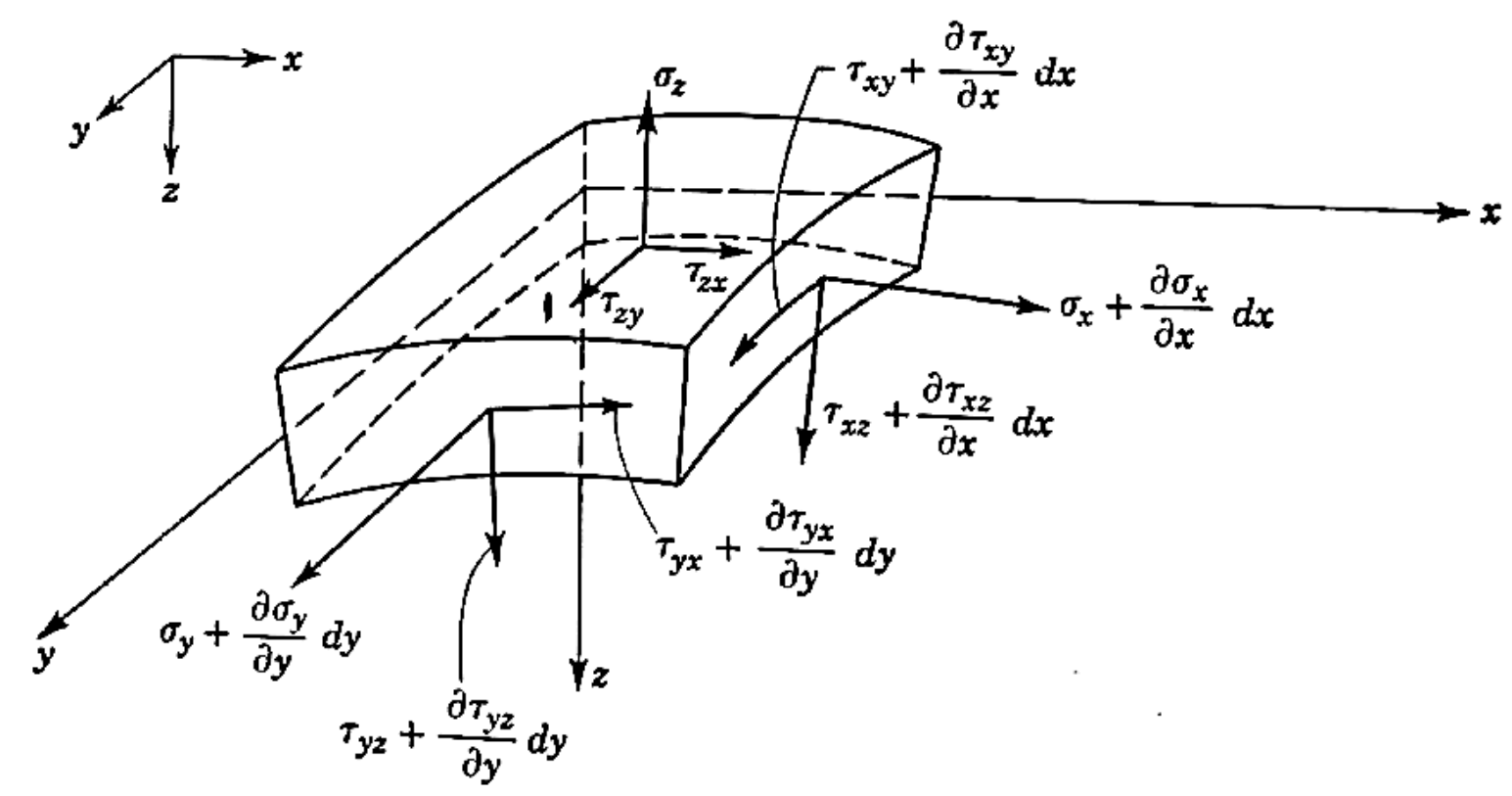

Figure 1. Stresses in a shell differential element (Adapted from Billington, 1965)

The third postulate of Love's theory is an extension of the Kirchhoff's beam theory. In this case it similarly states that straight lines that are normal to the middle surface remain 
normal during the deformation, to this effect, the in-plane displacements should only vary linearly through the thickness, and therefore, the normal strain components should be neglected in the formulation. To comply with this assumption in the formulation, the following relation should be satisfied:

$$
\varepsilon_{z}=\gamma_{\mathrm{xz}}=\gamma_{\mathrm{yz}}=0
$$

Finally, consistent with the first assumption, the forth postulate proposes that the transverse normal stress is negligible as well:

$$
\sigma_{\mathrm{z}}=0
$$

The resulting constitutive equations proposed by Love's theory, are summarized as follows:

$$
\begin{gathered}
\varepsilon_{\mathrm{x}}=\frac{\sigma_{\mathrm{x}}}{E_{\mathrm{x}}}-\frac{v_{\mathrm{xy}}}{E_{\mathrm{y}}} \sigma_{\mathrm{y}} \\
\varepsilon_{\mathrm{y}}=\frac{\sigma_{\mathrm{y}}}{E_{\mathrm{y}}}-\frac{v_{\mathrm{yx}}}{E_{\mathrm{x}}} \sigma_{\mathrm{x}} \\
\gamma_{\mathrm{xy}}=\frac{\tau_{\mathrm{xy}}}{G_{\mathrm{xy}}}, \quad \varepsilon_{\mathrm{z}}=\gamma_{\mathrm{xz}}=\gamma_{\mathrm{yz}}=\sigma_{\mathrm{z}}=0
\end{gathered}
$$

In order to use this theory, as mentioned before, the shell should be classified as a thin shell. Since the author did not specify the definition of a thin shell, numerous limitations were later established by different other researchers which were not in most cases quite in line with each other. However, in the absence of a precise definition, Kraus (1967) recommended as a general rule, to apply this theory only to shells with a thickness smaller than $1 / 10$ of the main radius of curvature. 
Later, after its publication, the theory received numerous critiques since multiple assumptions were made to simplify the formulation and some inconsistencies were also found in the postulates. Therefore, further investigations were later developed to create more accurate formulations; however, most of them were also based on similar postulates, differing from each other in the kind of assumptions made.

\subsection{Governing equations of general linear shell theory}

According to Billington (1965), to define the general formulation of shells, it is necessary to follow the following five steps:

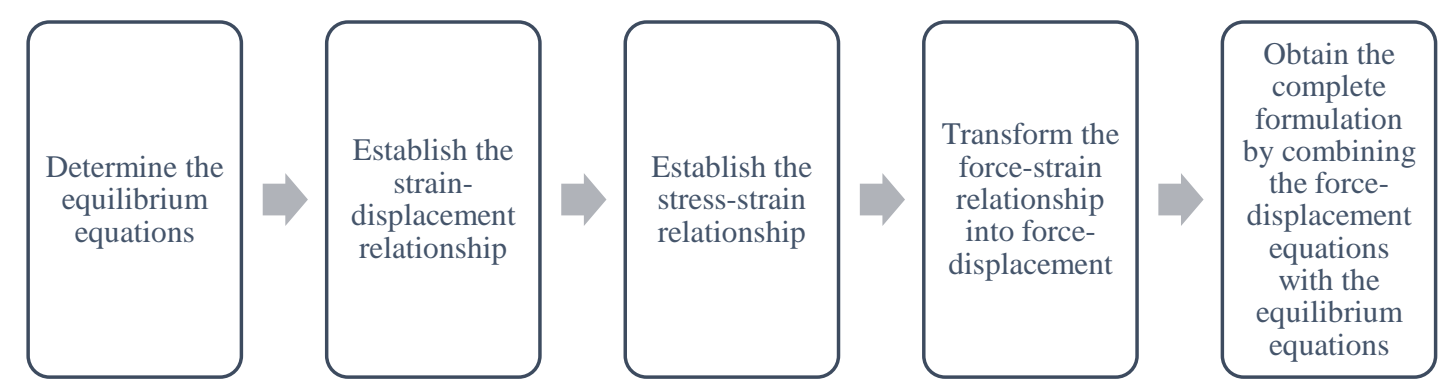

As stated by Billington, in accordance with the differential element in Figure 2, the equilibrium forces should be established with the following equations:
$\Sigma \mathrm{X}=0$
$\Sigma \mathrm{Mx}=0$
$\Sigma \mathrm{Y}=0$
$\Sigma \mathrm{My}=0$
$\Sigma \mathrm{Z}=0$
$\Sigma \mathrm{Mz}=0$ 


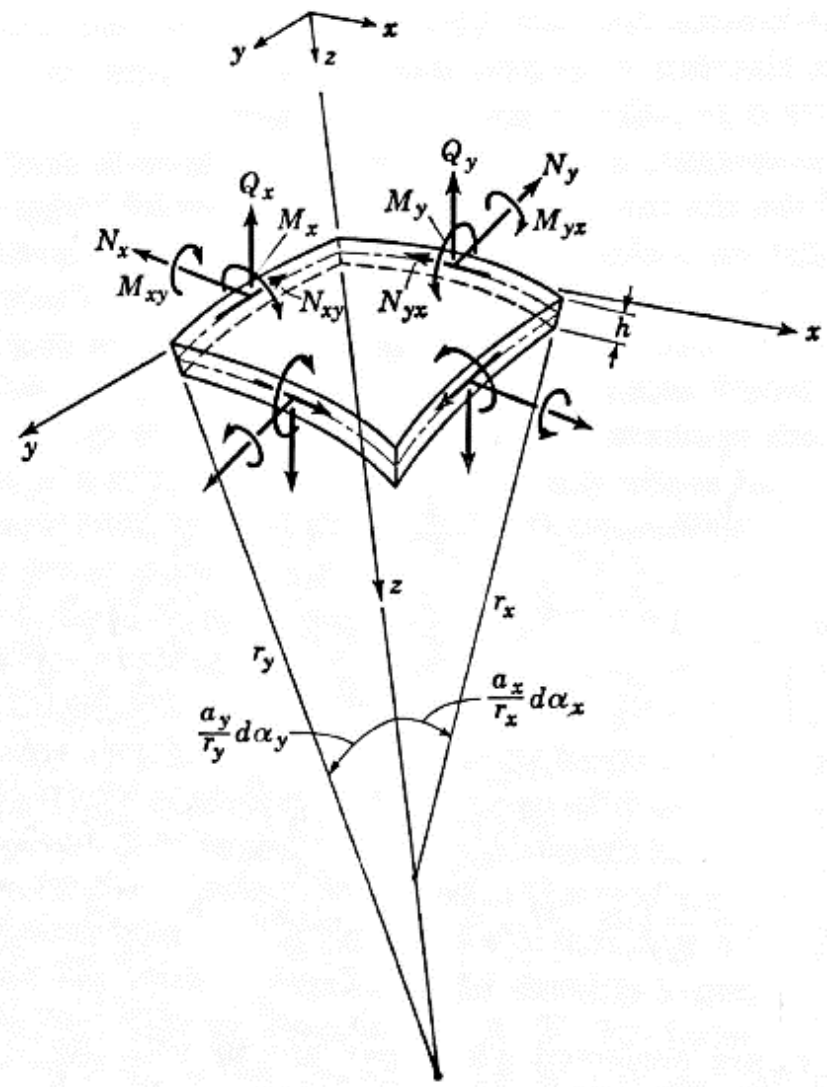

Figure 2. Differential Shell element (Adapted from Billington, 1965)
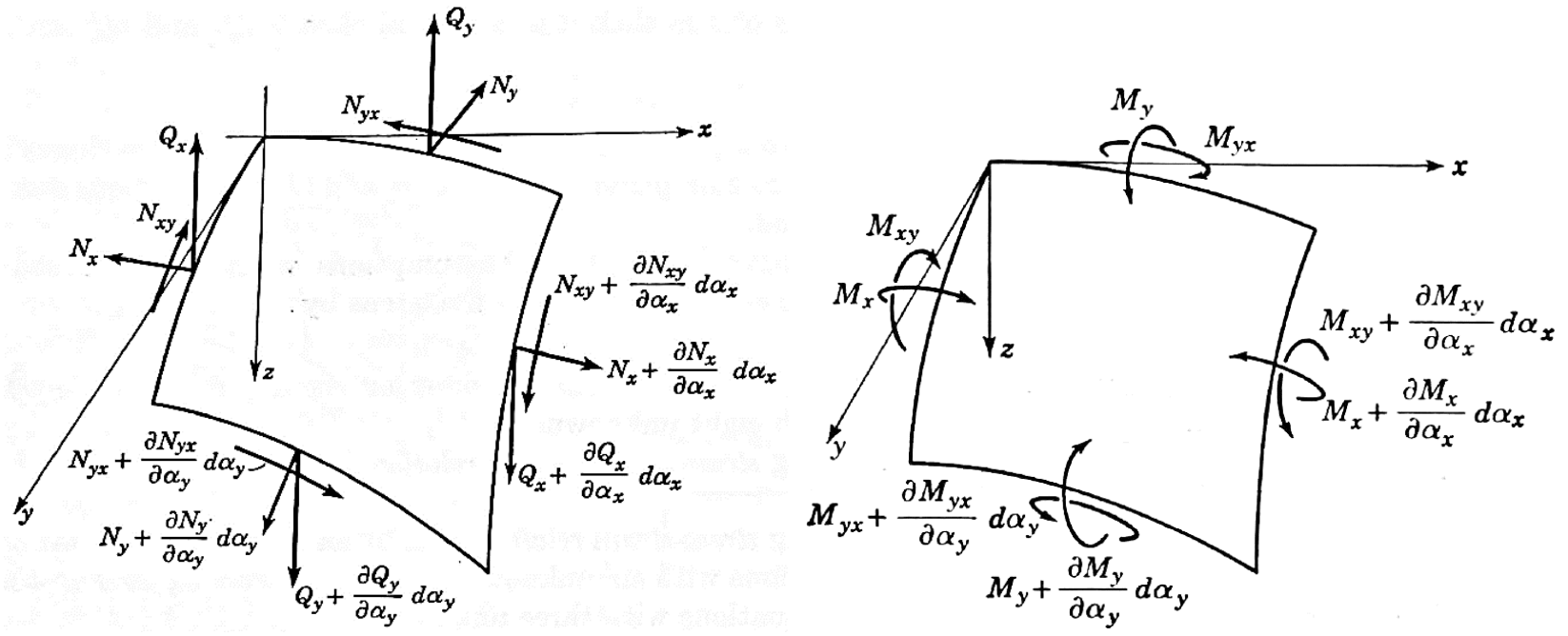

Figure 3. Stress resultants (Adapted from Billington, 1965) 
Forces and moments acting on the differential element are described as resultant stresses $\left(N_{x}, N_{y}, N_{x y}, N_{y x}, Q_{x}\right.$ and $\left.Q_{y}\right)$ and stress couples $\left(M_{x}, M_{y}, M_{y x}\right.$ and $\left.M_{x y}\right)$ acting in the middle surface (Figure 3), as integrals of stress over the shell thickness (h):

$$
\begin{aligned}
N_{x}=\int_{-h / 2}^{h / 2} \sigma_{x}\left(1-\frac{z}{r_{y}}\right) d z & N_{y}=\int_{-h / 2}^{h / 2} \sigma_{y}\left(1-\frac{z}{r_{x}}\right) d z \\
N_{x y}=\int_{-h / 2}^{h / 2} \tau_{x y}\left(1-\frac{z}{r_{y}}\right) d z & N_{y x}=\int_{-h / 2}^{h / 2} \tau_{y x}\left(1-\frac{z}{r_{x}}\right) d z \\
Q_{x}=\int_{-h / 2}^{h / 2} \tau_{x z}\left(1-\frac{z}{r_{y}}\right) d z & Q_{y}=\int_{-h / 2}^{h / 2} \tau_{y z}\left(1-\frac{z}{r_{x}}\right) d z \\
M_{x}=\int_{-h / 2}^{h / 2} \sigma_{x}\left(1-\frac{z}{r_{y}}\right) d z & M_{y}=\int_{-h / 2}^{h / 2} \sigma_{y}\left(1-\frac{z}{r_{x}}\right) d z \\
M_{x y}=-\int_{-h / 2}^{h / 2} \tau_{x y}\left(1-\frac{z}{r_{y}}\right) d z & M_{y x}=\int_{-h / 2}^{h / 2} \tau_{y x}\left(1-\frac{z}{r_{x}}\right) d z
\end{aligned}
$$

Since the shell thickness is assumed to be small in comparison with the other dimensions, the terms $\frac{z}{r_{y}}$ and $\frac{z}{r_{x}}$ are neglected from the formulations when they appear with unity. In the same way, due to the simplifications stated above for thin shells, $\tau_{x y}$ is expected to be equal to $\tau_{y x}$; therefore:

$$
\begin{gathered}
N_{x y}=N_{y x} \\
M_{x y}=-M_{y x}
\end{gathered}
$$

As a result of the above mentioned assumptions and considering the equilibrium 
conditions, the equation $\Sigma \mathrm{Mz} \neq 0$ can be therefore dropped from the system. The complete formulation is derived by Billington (1965) which results in a system with five equations and eight unknowns, as follows:

$$
\begin{array}{r}
\frac{\partial}{\partial \alpha_{x}}\left(N_{x} a_{y}\right)-N_{y} \frac{\partial a_{y}}{\partial \alpha_{x}}+N_{x y} \frac{\partial a_{x}}{\partial \alpha_{y}}+\frac{\partial}{\partial \alpha_{y}}\left(N_{y x} a_{x}\right)-Q_{y} \frac{a_{x} a_{y}}{\mathrm{r}_{x y}}-Q_{x} \frac{a_{x} a_{y}}{\mathrm{r}_{x}}+p_{x} a_{x} a_{y}=0 \\
\frac{\partial}{\partial \alpha_{y}}\left(N_{y} a_{x}\right)-N_{x} \frac{\partial a_{x}}{\partial \alpha_{y}}+N_{y x} \frac{\partial a_{y}}{\partial \alpha_{x}}+\frac{\partial}{\partial \alpha_{x}}\left(N_{x y} a_{y}\right)-Q_{x} \frac{a_{x} a_{y}}{\mathrm{r}_{x y}}-Q_{y} \frac{a_{x} a_{y}}{\mathrm{r}_{y}}+p_{y} a_{x} a_{y}=0 \\
\frac{\partial}{\partial \alpha_{x}}\left(Q_{x} a_{y}\right)+\frac{\partial}{\partial \alpha_{x}}\left(Q_{y} a_{x}\right)+N_{x} \frac{a_{x} a_{y}}{\mathrm{r}_{x}}+N_{x y} \frac{a_{x} a_{y}}{\mathrm{r}_{x y}}+N_{y x} \frac{a_{x} a_{y}}{\mathrm{r}_{x y}}+N_{y} \frac{a_{x} a_{y}}{\mathrm{r}_{y}}+p_{z} a_{x} a_{y}=0 \\
-\frac{\partial}{\partial \alpha_{y}}\left(M_{y} a_{x}\right)+M_{x} \frac{\partial a_{x}}{\partial \alpha_{y}}-M_{y x} \frac{\partial a_{y}}{\partial \alpha_{x}}+\frac{\partial}{\partial \alpha_{x}}\left(M_{x y} a_{y}\right)+Q_{y} a_{x} a_{y}=0 \\
-\frac{\partial}{\partial \alpha_{x}}\left(M_{x} a_{y}\right)+M_{y} \frac{\partial a_{y}}{\partial \alpha_{x}}+M_{x y} \frac{\partial a_{x}}{\partial \alpha_{y}}-\frac{\partial}{\partial \alpha_{y}}\left(M_{y x} a_{x}\right)+Q_{x} a_{x} a_{y}=0
\end{array}
$$

Where, $p_{x}, p_{y}$, and $p_{z}$ represent the external loads applied on the shell, $\alpha_{x}$ and $\alpha_{y}$ are curvilinear coordinates along the respective sides, $a_{x}$ and $a_{y}$ are the Lamé parameters representing the changes in the arc length of the surface and $r_{x}$ and $r_{y}$ are the principal radius of curvature as shown in Figure 2.

The next step establishes the strain - displacement relationships. Based on Figure 4, the displacements are proposed as follows: 

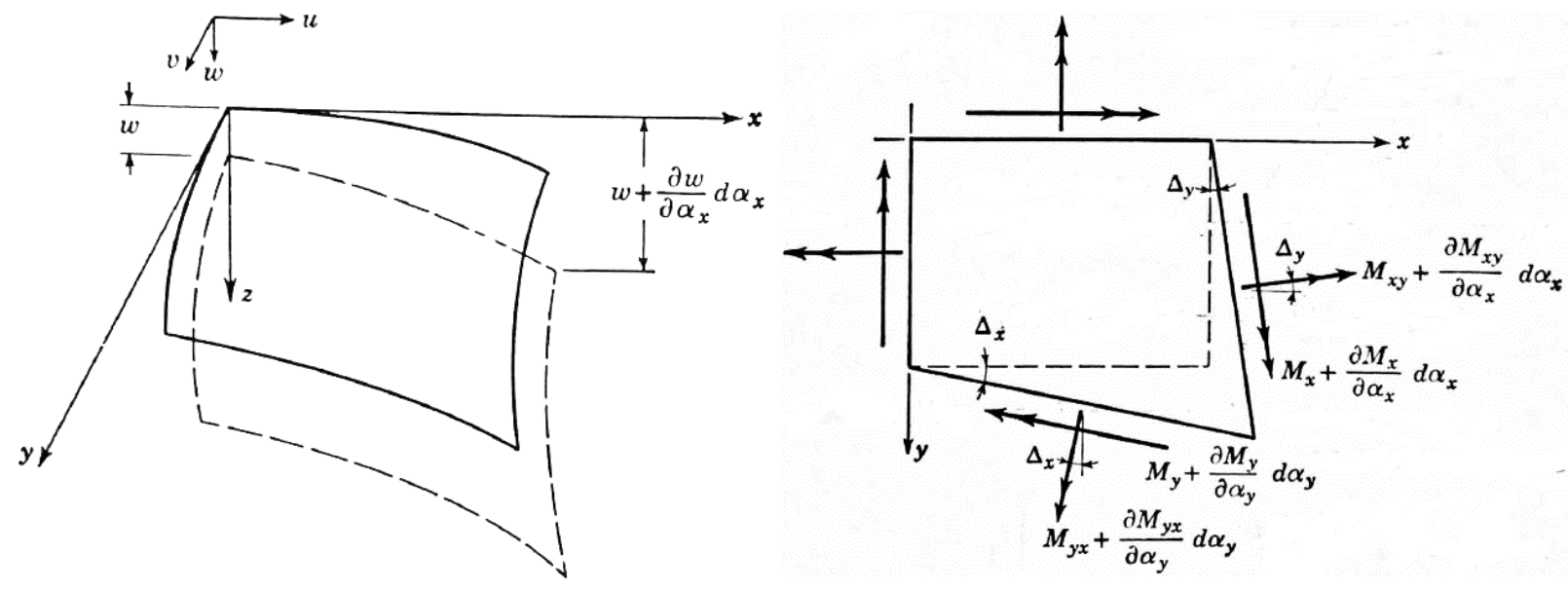

Figure 4. Shell element after deformation (Adapted from Billington, 1965)

$$
\begin{gathered}
\varepsilon_{\mathrm{x} 0}=\frac{1}{a_{\mathrm{x}}} \frac{\partial \mathrm{u}}{\partial \alpha_{x}}+\frac{v}{a_{\mathrm{x}} a_{\mathrm{y}}} \frac{\partial a_{\mathrm{x}}}{\partial \alpha_{y}}-\frac{w}{r_{\mathrm{x}}} \\
\varepsilon_{\mathrm{y} 0}=\frac{1}{a_{\mathrm{y}}} \frac{\partial \mathrm{v}}{\partial \alpha_{y}}+\frac{u}{a_{\mathrm{x}} a_{\mathrm{y}}} \frac{\partial a_{\mathrm{y}}}{\partial \alpha_{x}}-\frac{w}{r_{\mathrm{y}}} \\
\gamma_{\mathrm{xy} 0}=\frac{1}{a_{\mathrm{x}}} \frac{\partial \mathrm{v}}{\partial \alpha_{x}}+\frac{1}{a_{\mathrm{y}}} \frac{\partial \mathrm{u}}{\partial \alpha_{y}}-\frac{u}{a_{\mathrm{x}} a_{\mathrm{y}}} \frac{\partial a_{\mathrm{x}}}{\partial \alpha_{y}}-\frac{v}{a_{\mathrm{x}} a_{\mathrm{y}}} \frac{\partial a_{\mathrm{y}}}{\partial \alpha_{x}}-\frac{2 w}{r_{\mathrm{xy}}} \\
\mathrm{X}_{\mathrm{x}}=\frac{1}{a_{\mathrm{x}}} \frac{\partial \emptyset_{x}}{\partial \alpha_{x}}+\frac{\emptyset_{y}}{a_{\mathrm{x}} a_{\mathrm{y}}} \frac{\partial a_{\mathrm{x}}}{\partial \alpha_{y}} \\
\mathrm{X}_{\mathrm{y}}=\frac{1}{a_{\mathrm{y}}} \frac{\partial \emptyset_{y}}{\partial \alpha_{y}}+\frac{\emptyset_{x}}{a_{\mathrm{x}} a_{\mathrm{y}}} \frac{\partial a_{\mathrm{y}}}{\partial \alpha_{x}} \\
\frac{1}{a_{\mathrm{y}}} \frac{\partial \emptyset_{x}}{\partial \alpha_{y}}+\frac{1}{a_{\mathrm{x}}} \frac{\partial \emptyset_{y}}{\partial \alpha_{x}}-\frac{\emptyset_{x}}{a_{\mathrm{x}} a_{\mathrm{y}}} \frac{\partial a_{\mathrm{x}}}{\partial \alpha_{y}}-\frac{\emptyset_{y}}{a_{\mathrm{x}} a_{\mathrm{y}}} \frac{\partial a_{\mathrm{y}}}{\partial \alpha_{x}}
\end{gathered}
$$

Where, the components of displacement in the $\mathrm{x}, \mathrm{y}$ and $\mathrm{z}$ direction are taken as $\mathrm{u}, \mathrm{v}$ and $\mathrm{w}$, respectively; $\emptyset_{x}$ and $\emptyset_{y}$ represent the rotation of the middle surface, $\varepsilon_{\mathrm{x} 0}$ and $\varepsilon_{\mathrm{y} 0}$ are 
the strains in the corresponding direction and $\gamma_{\mathrm{xy} 0}$ is the angular shear strain. Finally, the last three formulations $\left(\mathrm{X}_{\mathrm{x}}, \mathrm{X}_{\mathrm{y}}\right.$ and $\left.2 \mathrm{X}_{\mathrm{xy}}\right)$ represent the strains due to bending, defined as changes in the curvature of the element.

On the other hand, the deformation of the shell neglecting the small terms is expressed according to the following equations:

$$
\begin{gathered}
\varepsilon_{\mathrm{x}}=\varepsilon_{\mathrm{x} 0}-z X_{\mathrm{x}} \\
\varepsilon_{\mathrm{y}}=\varepsilon_{\mathrm{y} 0}-z X_{\mathrm{y}} \\
\gamma_{\mathrm{xy}}=\gamma_{\mathrm{xy} 0}-2 z X_{\mathrm{xy}}
\end{gathered}
$$

This deformation is constituted by the strain due to the change in curvature and the strain caused by the extension of the middle surface.

Assuming a linear elastic, isotropic and homogenous material; and adopting Hooke's law; the stress-strain relationship could be defined as follows:

$$
\begin{gathered}
\sigma_{\mathrm{x}}=\frac{\mathrm{E}}{1-v^{2}}\left(\varepsilon_{x}+v \varepsilon_{y}\right) \\
\sigma_{\mathrm{y}}=\frac{\mathrm{E}}{1-v^{2}}\left(\varepsilon_{y}+v \varepsilon_{x}\right) \\
\tau_{\mathrm{xy}}=\frac{\mathrm{E}}{2(1+v)} \gamma_{\mathrm{xy}}
\end{gathered}
$$

From the above equation and the forces described in equation 6 and 7, the resulting forces could be rewritten as: 


$$
\begin{array}{cc}
N_{x}=K\left(\varepsilon_{1}+v \varepsilon_{2}\right) & N_{y}=K\left(\varepsilon_{2}+v \varepsilon_{1}\right) \\
M_{x}=-D\left(\mathrm{X}_{x}+v \mathrm{X}_{y}\right) & M_{y}=-D\left(\mathrm{X}_{y}+v \mathrm{X}_{x}\right) \\
N_{x y}=N_{y x}=\gamma \mathrm{Gh} & M_{x y}=-M_{y x}=D(1-v) \mathrm{X}_{x y}
\end{array}
$$

Where:

$$
\begin{gathered}
K=\frac{\mathrm{E}}{1-v^{2}} \\
G=\frac{\mathrm{E}}{2(1+v)} \\
D=\frac{\mathrm{Eh}^{3}}{12\left(1-v^{2}\right)}
\end{gathered}
$$

In the above formulations, $\mathrm{E}$ is the modulus of elasticity, $\mathrm{K}$ is the extensional rigidity, $\mathrm{G}$ is the shear modulus and $\mathrm{D}$ is the bending rigidity of the shell.

Finally, the force-displacement relationship is obtained from the strain-displacement equations and the resulting forces described above, as:

$$
\begin{aligned}
& N_{x}=K\left[\frac{1}{a_{\mathrm{x}}} \frac{\partial \mathrm{u}}{\partial \alpha_{x}}+\frac{v}{a_{\mathrm{x}} a_{\mathrm{y}}} \frac{\partial a_{\mathrm{x}}}{\partial \alpha_{y}}-\frac{w}{r_{\mathrm{x}}}+v\left(\frac{1}{a_{\mathrm{y}}} \frac{\partial \mathrm{v}}{\partial \alpha_{y}}+\frac{u}{a_{\mathrm{x}} a_{\mathrm{y}}} \frac{\partial a_{\mathrm{y}}}{\partial \alpha_{x}}-\frac{w}{r_{\mathrm{y}}}\right)\right] \\
& N_{y}=K\left[\frac{1}{a_{\mathrm{y}}} \frac{\partial \mathrm{v}}{\partial \alpha_{y}}+\frac{u}{a_{\mathrm{x}} a_{\mathrm{y}}} \frac{\partial a_{\mathrm{y}}}{\partial \alpha_{x}}-\frac{w}{r_{\mathrm{y}}}+v\left(\frac{1}{a_{\mathrm{x}}} \frac{\partial \mathrm{u}}{\partial \alpha_{x}}+\frac{v}{a_{\mathrm{x}} a_{\mathrm{y}}} \frac{\partial a_{\mathrm{x}}}{\partial \alpha_{\mathrm{y}}}-\frac{w}{r_{\mathrm{x}}}\right)\right]
\end{aligned}
$$




$$
\begin{gathered}
N_{x y}=N_{y x}=\operatorname{Gh}\left(\frac{1}{a_{\mathrm{x}}} \frac{\partial \mathrm{v}}{\partial \alpha_{x}}+\frac{1}{a_{\mathrm{y}}} \frac{\partial \mathrm{u}}{\partial \alpha_{y}}-\frac{u}{a_{\mathrm{x}} a_{\mathrm{y}}} \frac{\partial a_{\mathrm{x}}}{\partial \alpha_{y}}-\frac{v}{a_{\mathrm{x}} a_{\mathrm{y}}} \frac{\partial a_{\mathrm{y}}}{\partial \alpha_{x}}-\frac{2 w}{r_{\mathrm{xy}}}\right) \\
M_{x}=-D\left[\frac{1}{a_{\mathrm{x}}} \frac{\partial \emptyset_{x}}{\partial \alpha_{x}}+\frac{\emptyset_{y}}{a_{\mathrm{x}} a_{\mathrm{y}}} \frac{\partial a_{\mathrm{x}}}{\partial \alpha_{y}}+v\left(\frac{1}{a_{\mathrm{y}}} \frac{\partial \emptyset_{y}}{\partial \alpha_{y}}+\frac{\emptyset_{x}}{a_{\mathrm{x}} a_{\mathrm{y}}} \frac{\partial a_{\mathrm{y}}}{\partial \alpha_{x}}\right)\right] \\
M_{y}=-D\left[\frac{1}{a_{\mathrm{y}}} \frac{\partial \emptyset_{y}}{\partial \alpha_{y}}+\frac{\emptyset_{x}}{a_{\mathrm{x}} a_{\mathrm{y}}} \frac{\partial a_{\mathrm{y}}}{\partial \alpha_{x}}+v\left(\frac{1}{a_{\mathrm{x}}} \frac{\partial \emptyset_{x}}{\partial \alpha_{x}}+\frac{\emptyset_{y}}{a_{\mathrm{x}} a_{\mathrm{y}}} \frac{\partial a_{\mathrm{x}}}{\partial \alpha_{y}}\right)\right] \\
M_{x y}=-M_{y x}=\frac{D(1-v)}{2}\left(\frac{1}{a_{\mathrm{y}}} \frac{\partial \emptyset_{x}}{\partial \alpha_{y}}+\frac{1}{a_{\mathrm{x}}} \frac{\partial \emptyset_{y}}{\partial \alpha_{x}}-\frac{\emptyset_{x}}{a_{\mathrm{x}} a_{\mathrm{y}}} \frac{\partial a_{\mathrm{x}}}{\partial \alpha_{y}}-\frac{\emptyset_{y}}{a_{\mathrm{x}} a_{\mathrm{y}}} \frac{\partial a_{\mathrm{y}}}{\partial \alpha_{x}}\right)
\end{gathered}
$$

These six equations along with the five equations obtained from the equilibrium condition provide a system of eleven equations and eleven unknowns. Since the number of equations is equal to the number of unknowns, the system has a unique solution. However, the large number of unknowns makes the problem difficult to solve that is usually addressed by making even further assumptions to simplify the formulation.

\subsection{Definition of spherical shells}

Shell structures can be found in many different geometric configurations. To this effect, they could be classified according to the shape of its middle surface as: cylindrical shells, spherical shells, barrel shells, folded plates, hyperbolic paraboloids, elliptic paraboloids and non-geometric shapes.

Along with the evolution of different shell configurations, some specialized theories were also proposed for each particular case. Although all these elements represent shell structures, each one has a particular behavior derived from its specific geometry. Therefore, this investigation focuses its attention on the particular case of spherical shells, also known as dome structures.

Domes are structures in the form of surfaces of revolution that have circular plans; 
they are usually constructed of a continuous rigid material such as reinforced concrete, steel or short linear elements, as in the case of geodesic domes. As a surface of revolution, its shape is created by rotating a curve name "meridian" about an axis of rotation called "shell axis". As shown in Figure 5, the curves created by the intersection of a horizontal plane with the surface are called "Parallels".

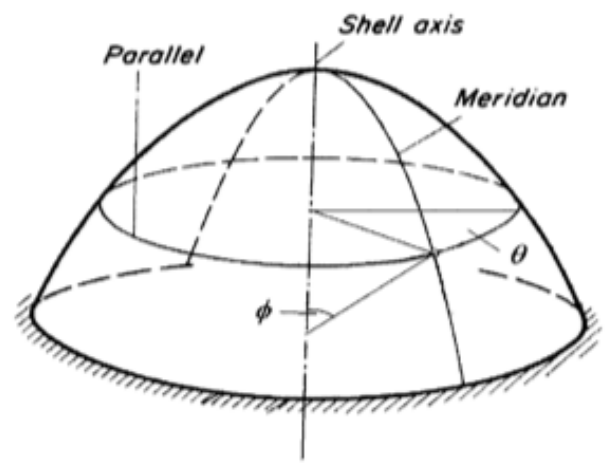

Figure 5. Surface of revolution (Adapted from Farnsworth Jr., 1998)

As any other shell structures, domes are able to carry the applied loads by developing a combination of membrane stresses and bending moments. However, thanks to the geometrical configuration of these structures, the membrane stresses are the main source of resistance and in most cases shear stresses and bending moments are negligible.

Ideally, the behavior of spherical shells could be represented by a uniform load applied throughout the surface. In this case the shell balances the forces by means of inplane tensile and compressive stresses without any bending moments; this defines a stress condition commonly known as membrane state of stresses.

Under this condition, according to Farnsworth Jr. (1998), the performance of the structure is ruled by two main types of stress: Meridional and Hoop. The first one similar to the case of an arch (Figure 6), experiences compression along the length with no bending moments developed as the applied force is assumed uniform; while the second one represents the stress acting on the horizontal plane. Even if they cannot resist vertical 
loading, they are able to restrain the out of plane movement of the meridians, contributing further to the resistance of the dome to bending moments.
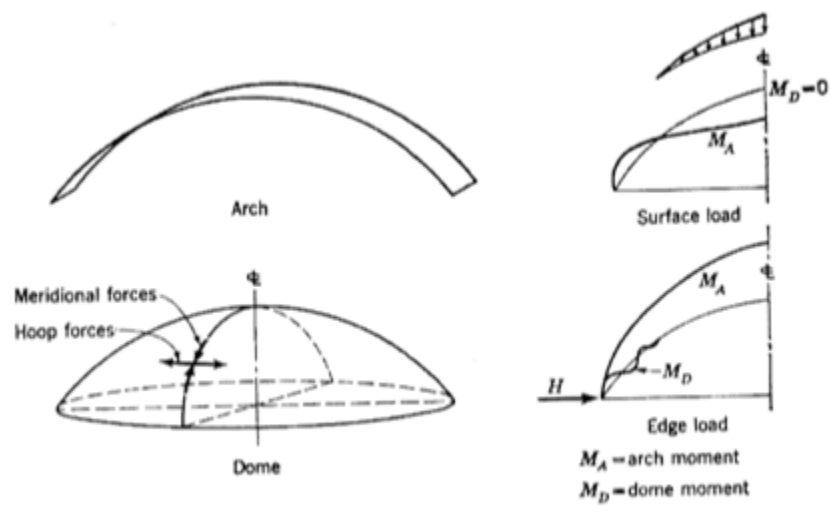

Figure 6. Dome vs arch (Adapted from Farnsworth Jr., 1998)

This will ideally result in an efficient and economical design providing a designer with a perfect platform to accomplish a safe and satisfactory structural performance when dealing with spherical shell structures. However, even if this state is ideal sometimes the edge and load circumstances do not always perform as desired and hence bending moments are inevitable in the structure.

This behavior usually takes place near the edges due to the supporting conditions, since at this point the structure is restricted to expand bending moments have to be developed. However, these are only formed in the surroundings of the disturbance region and as one moves away from it, the shell resumes its membrane action. Therefore, the major part of the structure is expected to behave as a membrane with no moment acting.

\subsection{Theory of elastic stability}

As stated earlier, shell structures present a particular behavior that makes them one of the most resistant structures; however, ironically, the more efficient the structure is with regards to its shape, the worse its mode of failure is expected to be, since, the type of failure 
they experience no longer represents a material resistance problem but an instability problem instead that is primarily influenced by their stiffness. Therefore, when designing these structures, special attention should be given to this matter, since this is not a progressive but a sudden failure usually leading to a total collapse.

This stability problem was compared with a simple case of equilibrium of a ball by S. Timoshenko and Gere (1961), yielding to the definition of three types of equilibrium states: the first one (Figure 7.a) is called "stable equilibrium", which occurs when an external perturbation is applied to the structure, in this case the ball, and after some oscillations it is able to return to its original position. Timoshenko, deduces with this, that if a structure is under the action of loads smaller than the critical one, it is considered as a stable structure.

The second proposed condition is represented by Figure 7.b. In this case if any small perturbation is applied to the structure, it will cause a permanent displacement. This situation represents a limit point for instability known as the critical load which once exceeded the structure becomes unstable experiencing large deflections and a subsequent failure. This condition receives the name of "unstable equilibrium". The third one represents a limit between the last two, called "indifferent or neutral equilibrium" (Figure 7.c).

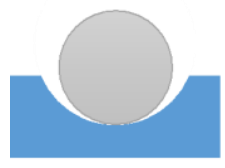

(a)

Stable equilibrium

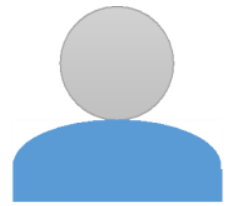

(b)

Neutral equilibrium

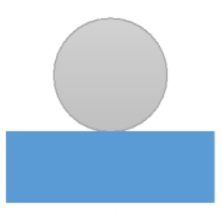

(c)

Unstable

Figure 7. Equilibrium of a system

The kind of failure described above during the unstable equilibrium condition is commonly known as buckling and is the main cause of failure of spherical shell structures. However, in reality the instability problem of spherical shells faces much deeper difficulties due to the wide variety of parameters that influence this type of failure as well as the mathematical challenges involved in their formulation. 
Therefore, in order to provide a better understanding of this problem, the buckling problem of spherical shells is further described by Ventsel (2001), using Figure 8.

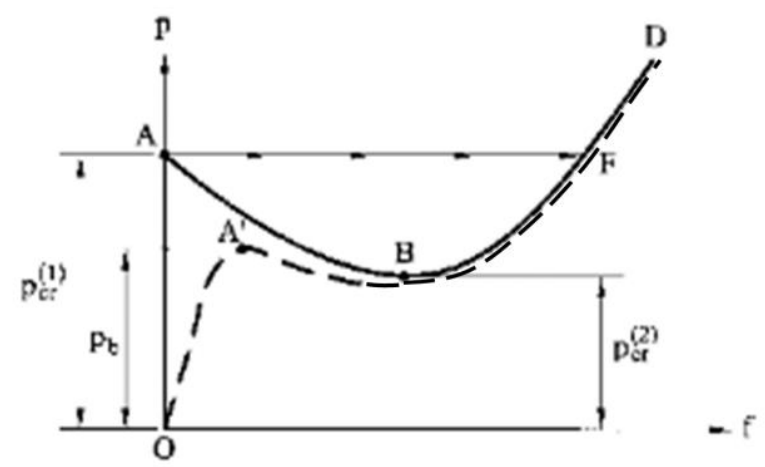

Figure 8. Buckling failure of shell structures (Adapted from Ventsel et al., 2001)

In Figure 8, OABD represents a perfect shell with no material or geometric nonlinearities. The first branch $(\mathrm{OA})$ shows that when perturbations $(\mathrm{P})$ below the critical load are applied to the structure, it behaves in a membrane state of stresses. Therefore, no deflections (f) are found at this stage. However, once it reaches the critical point (A) the structure loses its equilibrium state until point $\mathrm{F}$ where it retakes its stability. This type of instability is known as bifurcation buckling.

However, in reality structures are not perfect and no matter how meticulous the construction process is, they will always have some imperfections in their geometry that will cause imposed pre-buckling deformations and consequently a decrease in the capacity, in addition to material nonlinearity effects that can also cause a dramatic decrease in the capacity of this type of structure. Therefore, the real critical load is in fact much lower than the one specified for a perfect structure.

Considering this imperfect state in shells, a modified curve indicated as OA'BD in Figure 8 is suggested. As can be noticed, from the beginning the structure experiences some deviation from the original shape but still performing under the stable equilibrium state along the first branch $\left(\mathrm{OA}^{\prime}\right)$. However, this time the critical load is reached at a lower value $\left(\mathrm{A}^{\prime}\right)$, 
from this point the structure experience an unstable state of equilibrium until it reach a new stable configuration at the level of $\mathrm{A}^{\prime}$ on the branch BD. This type of failure is known as Snap-through buckling behavior.

According to the above graph, two limits for critical loads can be identified. First, is the load obtained from the bifurcation buckling analysis and could be defined as the "upper" limit critical load since it is the largest load that could be achieved from an instability analysis considering a perfect structure. Second, is the real buckling load denoted by point $\mathrm{A}^{\prime}$ on the graph which represents the point where an imperfect shell loses its equilibrium state.

This nonlinear buckling behavior is represented for spherical shells in Figure 9, where an excessive deformation is observed in the center of the structure; however, the failure shape that each structure experiences will be controlled by the type of loading applied, boundary conditions, size of imperfection, material nonlinearities, as well as its geometrical configuration.

\section{Plane of a Spherical Dome}
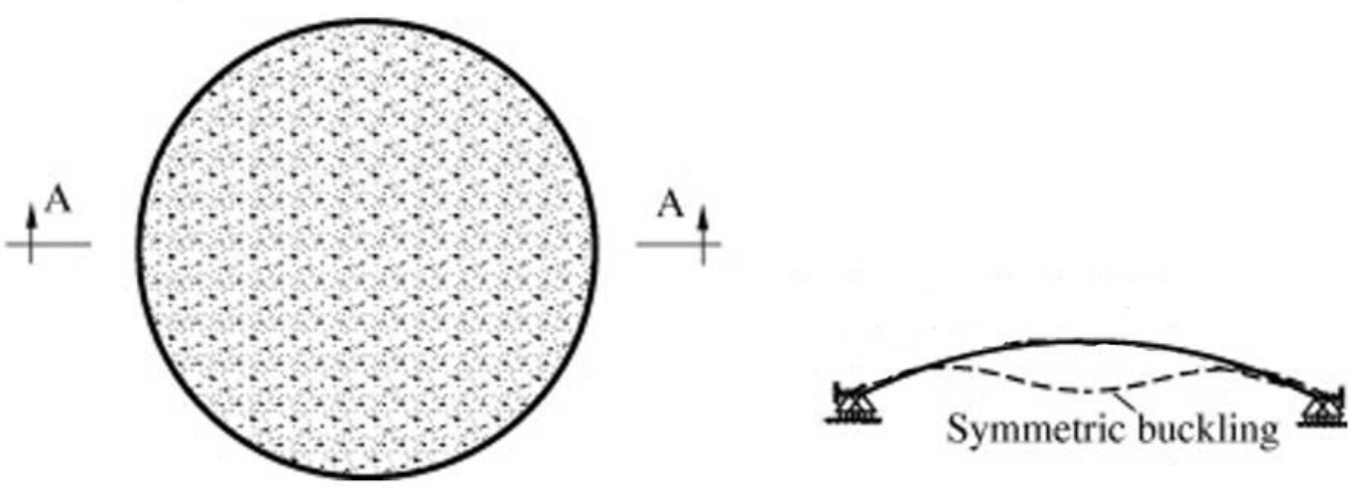

Figure 9. Buckling failure of a dome structure (Adapted from Hamed, 2010)

A deeper analysis into the factors influencing these two critical load values will be made later in this chapter. 


\subsection{History of buckling theories of thin spherical shells}

With the development of the theory of elastic stability came the definition of many formulations to analyze the buckling failure of different types of shells. The first one, is commonly known as the Classical buckling equation and is an elastic linear formulation that predicts the bifurcation buckling of a structure. This well-known theory was also proposed by Timoshenko (1936). This equation is based on the small deflection theory initially introduced by Love (1888) for thin shells in which, as explained before, all terms higher than quadratic were neglected in the energy equations resulting in a linear expression of the equilibrium state. This assumption along with the elastic stability theory result in the following equation for the buckling pressure $\left(q_{c r}\right)$ of axisymmetric spherical shells:

$$
q_{c r}=\frac{2 E h^{2}}{a^{2} \sqrt{3\left(1-v^{2}\right)}}
$$

Where $E$ and $v$ represents the corresponding modulus of elastic and Poisson's ratio of the material, $h$ the thickness of the spherical shell and $a$ corresponds to the main radius of curvature.

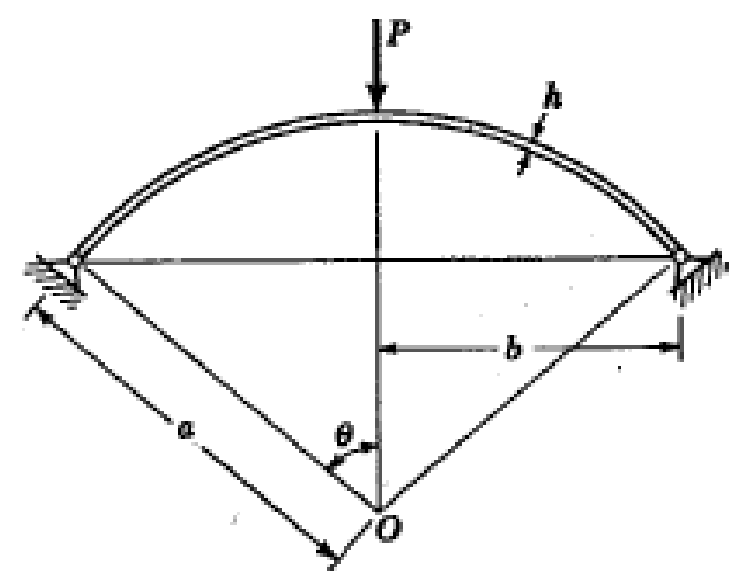

Figure 10. Geometry of spherical shell (Adapted from Timoshenko and Gere, 1961) 
While the magnitudes of the uniform compressive stress $(\sigma)$ and the critical stress $\left(\sigma_{c r}\right)$ for a spherical shell subjected to uniform external pressures were established as follows:

$$
\begin{gathered}
\sigma=\frac{q a}{2 h} \\
\sigma_{c r}=\frac{E h}{a \sqrt{3\left(1-v^{2}\right)}}
\end{gathered}
$$

As per this equation and assuming a Poisson's ratio $(v)$ of 0.17 for concrete, the critical buckling pressure was defined as follows:

$$
q_{c r}=1.17 E \frac{h^{2}}{R^{2}}
$$

Even though this equation was known as the classical formulation, for many years numerous experiments related to the buckling of shells showed a significant discrepancy between the experimental results and the ones obtained by this analytical approach.

One good example of this discrepancy was shown through an empirical research by Donnell (1934). In his investigation, he tested samples of cylindrical shells with different types of materials. It was observed that buckling occurred in values significantly lower than the ones found with this theoretical equation. He concluded that the deviation was highly influenced by the radius-to-thickness ratio; since, higher ratios were leading to higher errors.

According to Donnel, the formulation proposed by Timoshenko was analytically correct; however, the assumption of no initial imperfections was found to have considerable effect on the final results as a result of which good correlation with experimental results was rarely achieved. 
Some years later, Sechler and Bollay (1939) performed a similar experimental study but this time with spherical shells and observed that for this type of shells the buckling failure was taking place at values around $1 / 4$ of the theoretical values.

Another example of this discrepancy was indicated by Carlson et al. (1967), where 32 complete spherical shells were tested. In this investigation the results obtained from experiment were normalized with respect to those from the classical buckling equation and were plotted as a graph (Figure 11). As shown, most of the results were far from the classical buckling values; claiming again that this unexpected difference was the consequence of the imperfection in the tested models.

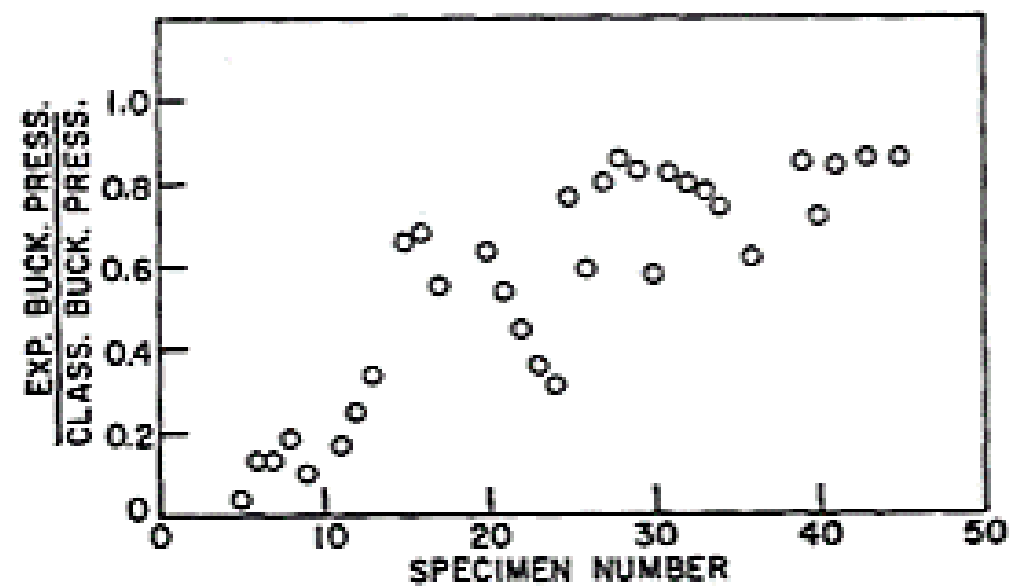

Figure 11. Summary of sphere-test performance (Adapted from Carlson et al., 1967)

Due to these discrepancies, Timoshenko and Gere (1961) later developed an empirical equation that according to them would result in satisfactory results for spherical shells having the following configurations:

$$
\begin{gathered}
400 \leq a / h \leq 2000 \\
20^{\circ} \leq \theta \leq 60^{\circ} .
\end{gathered}
$$




$$
q_{c r}=\left(1-0.175 \frac{\theta-20^{\circ}}{20^{\circ}}\right)\left(1-0.175 \frac{0.07^{a} / h}{400}\right)(0.3 E)\left(\frac{h}{a}\right)^{2}
$$

However, this formulation did not yet represent a general formulation for an accurate determination of the buckling capacity of this type of structures. As explained, only simple linear analytical and empirical formulations had been developed for many years for the purpose of analysis of this stability problem. It was not until Von Karman and Tsien (1939) proposed a solution to this problem using a different approach, this time by including the nonlinear terms in the equations of equilibrium. However, once again due to the complexity of the problem, they had to make some assumptions to simplify the formulation, which are summarized as follows:

- $\quad$ The buckled length is small.

- The deflection is rotationally symmetric.

- The deflection of any element of the shell is parallel to the axis of rotational symmetry.

- The effect of lateral contraction is neglected due to the assumption of Poisson's ratio equal to 0 .

$$
\begin{gathered}
\sigma_{c r}=0.183 \frac{E h}{R} \\
q_{c r}=0.366 E \frac{h^{2}}{R^{2}}
\end{gathered}
$$

As a result, the above equations were finally proposed. From this equation, it can be clearly noticed that the buckling pressure is remarkably lower than the one obtained by the classical equation. It is important to note that the values obtained from this formulation fit the previously mentioned experimental data very closely. 
According to Karman and Tsien (1939), the classical buckling theory could represent an upper limit to the buckling load that can be reached experimentally only if the structure is built with extreme precision and with no imperfections. However, since in reality achieving such level of perfection is considered impossible, the values are always expected to be close to the lower buckling load determined by their approach.

On the other hand, Huang (1963) compared the effects of having different types of boundary conditions in spherical caps. As observed in Figure 12, generally a simply supported shallow cap results in lower buckling pressures as compared to those with boundaries free to displace radially or clamped on the edge. However, one can note an exception, for the case of spherical caps with a shallowness parameter of 4 , as in this case the critical pressure is caused by the clamped base condition and not the other two.

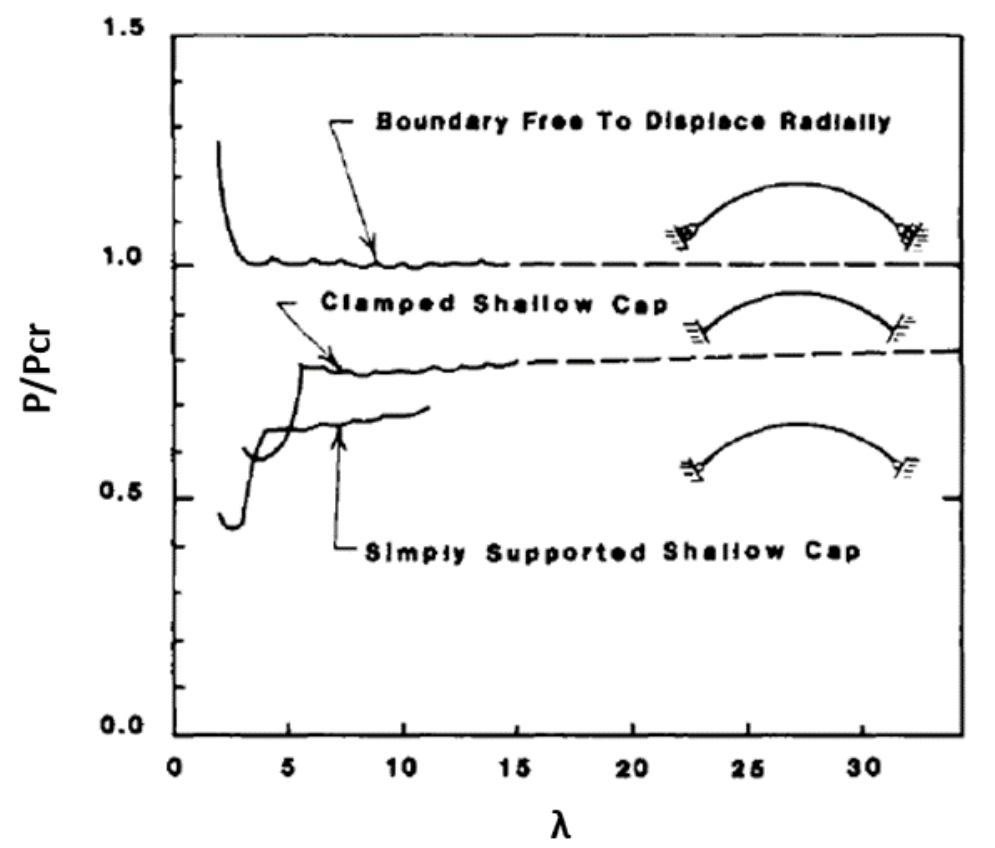

Figure 12. Comparison between different boundary conditions (Adapted from Huang, 1963)

As previously mentioned, the classical buckling equation is a general formulation that was originally developed for a complete spherical shell; however, in order to simplify the analysis of these elements, the problem was usually idealized as a spherical cap with clamped 
edges. Even if this seemed an adequate approach to represent the problem, the analysis of a spherical cap introduced a new parameter that was not considered in a complete sphere problem, the presence of edges. Driven by this and with the intention of analyzing the influence of the shallowness of the structure on the buckling failure, Bushnell (1981) proposed a new approach. He obtained the buckling pressure of spherical caps with different shallowness parameters $(\lambda)$ and compared it with the bifurcation buckling of complete spherical shells as shown in Figure 13. He concluded that the critical pressure of a spherical cap with $\lambda$ value lower than 7 was far from that of a complete shell. He observed that where $\lambda<3.5$ the structure does not show an instability failure and that for $\lambda$ about 4 , there is no bifurcation condition but a snap-through buckling instead. While for $\lambda$ greater than 7 , the prebuckling behavior is more linear resulting in values more similar to bifurcation buckling. However, even in deep spherical shells, the presence of edges always leads to values lower than those of the classical pressure equation.

From the previous investigations, it was concluded that the most critical buckling pressure was the one found for the case of a clamped boundary condition and a shallowness parameter of 4.

Krenzke and Kiernan (1965) studied the effect of initial imperfections on the collapse of spherical shells. They developed an empirical equation based on the classical pressure and the results obtained during their testing program, and concluded that the collapse should occur at around 0.7 times the classical pressure.

They further concluded that the collapse of spherical shells was primarily a local phenomenon and was therefore highly dependent on the local geometry, emphasizing with this the importance of the initial imperfections (unevenness factor). As per the proposed formulation, the sphere radius is expressed to the power of two and so any small change can lead to an important impact on the buckling capacity of the structure. 


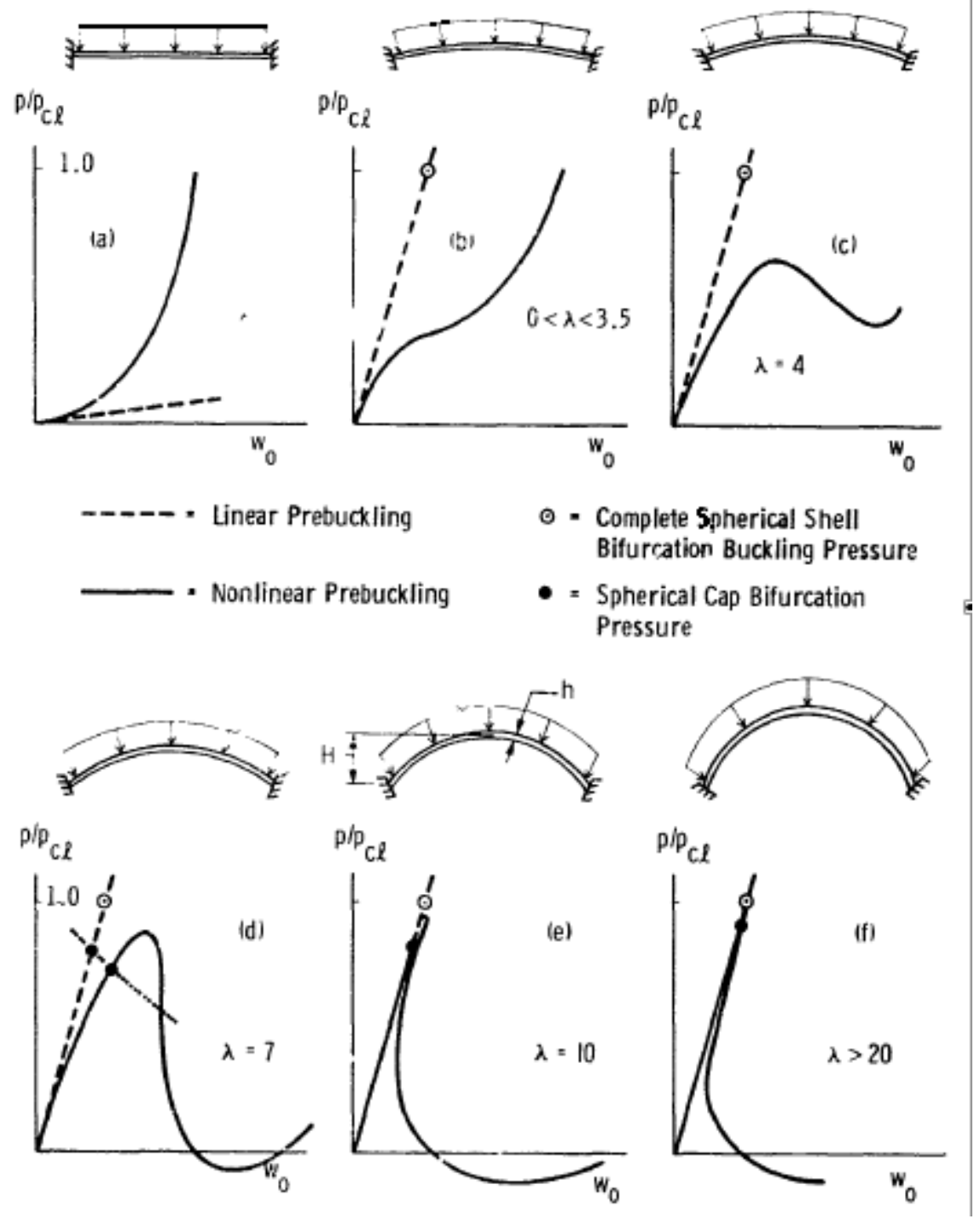

Figure 13. Load-deflection curves and bifurcation buckling of spherical caps with various values of the shallowness parameter (Adapted from Bushnell, 1981)

They summarized the effect of the initial imperfections as given in Figure 14. As shown, a perfect geometry leads to results close to that of the classical formulation; however, as the imperfection increases, the critical pressure decreases. 


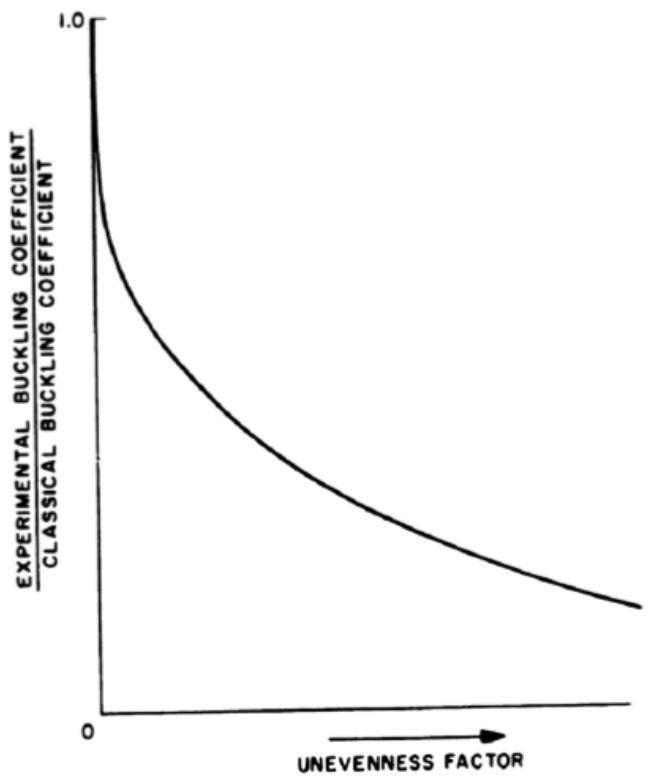

Figure 14. Effect of initial imperfections for $\boldsymbol{v}=0.3$ (Adapted from Krenzke and Kiernan, 1965)

In 1967, Bushnell determined the external pressures under which imperfect spherical shells would collapse. He noticed that buckling always took place in the shell zones with imperfection, concluding that the maximum load carrying capability of the structure corresponds to the maximum load that the imperfection can support.

However, it was not until 1983, when Zarghamee and Heger finally summarized the results of all the previous investigations into one simple formulation; adopting mainly the postulates proposed by Huang (1963), Krenzke and Kiernan (1965) and Bushnell (1967). In line with the results of previous research on concrete spherical shells, they stated that the main factor causing the discrepancy between the empirical and classic buckling equation results was the imperfection of the structure caused by instantaneous deformations, creep deformations and manufacturing tolerances. Based on this assumption, they determined the buckling strength of a concrete spherical shell by separately analyzing its imperfection; considering that the buckling strength of the entire structure was governed by the lowest value estimated for the buckling capacity of the imperfect region (Bushnell, 1967). To this effect, they picked for the size of the imperfection the least resistant configuration 
corresponding to a shallowness parameter of 4 clamped along the edge.

The results showed that such imperfection could reduce the buckling load to at least fifty percent of the buckling load of a perfect shell. As a result, a new reduction factor had to be included in the formulation, in order to take into account this important effect, as follows:

$$
\beta_{\text {imp }}=\left(R / R_{\text {imp }}\right)^{2}
$$

Where $\mathrm{R}$ represents the nominal radius of the perfect shell, while $\mathrm{R}_{\mathrm{imp}}$ describes the main radius of the imperfection area.

Zarghamee and Heger (1983) also studied the effect of material nonlinearities: cracking, stress-strain nonlinearity, and creep. The results showed that stress-strain nonlinearity did not have an important influence on the results, since the buckling usually occurs at nominal stresses far below yielding. In the same way, they concluded that flexural cracking was not expected to have a significant impact on the buckling of spherical shells either, as this is a localized phenomenon that does not produce significant reductions in the stiffness of the structure as a whole. In contrast, they concluded that creep could cause a drastic reduction in buckling capacity, since domes may undergo considerable creep deformation under sustained loads such as snow or other types of live load.

The importance of this effect lies in the fact that it changes the geometry of the structure and as previously mentioned, a small change in the radius of the dome can result in a significant reduction in its buckling capacity. For this reason, in order to take into account the creep effect, another parameter was also added to the formulation resulting in the following final equation: 


$$
\begin{aligned}
& P_{o}=0.66 \emptyset \beta_{c} \beta_{i m p} E \frac{t^{2}}{R^{2}} \\
& \beta_{\text {imp }}=\left\{\begin{array}{c}
0.5 \\
\left(R / R_{\text {imp }}\right)^{2} \quad \text { if } R_{\text {imp }}<1.4 R \\
\text { f } R_{\text {imp }} \geq 1.4 R
\end{array}\right. \\
& \beta_{c}=\left\{\begin{array}{cc}
0.476+(0.005 t-0.046)\left(1-\frac{L L}{12}\right) & \text { for } L L<12 \mathrm{psf} \\
0.44+0.003(\mathrm{LL}) & \text { for } 12 p s f \leq \mathrm{LL}<30 \mathrm{psf} \\
0.53 & \text { for } L L>30 \mathrm{psf}
\end{array}\right.
\end{aligned}
$$

Where $P_{o}$ is described as the buckling load of the dome incorporating the effects of geometric imperfections $\left(\beta_{i m p}\right)$ and creep, material nonlinearity and cracking $\left(\beta_{c}\right)$ as a function of the thickness ( $\mathrm{t}$ ) and live load (LL).

These previous formulations were verified by comparing the analytical results with Vandepitte and Rathe (1980) experimental results on microconcrete specimens, proving a general agreement with the test results.

Subsequently, this hypothesis was later supported by the work done by Hamed et al. (2011), who carried out experimental and theoretical analysis under short and long term conditions. Based on the results obtained, they concluded that in the cases where the structure was gradually loaded, the structure failed under the action of large forces and moderate displacements; however, when sustained loading was applied, this time the structures lost its stability by the combined effect of moderate loading and large deflections resulted from creep; affirming with this the relevance of creep on the buckling resistance of concrete spherical shells as stated by Zarghamee.

However, even if Zarghamee's theory provided accurate results; Strohman and Liepins (2009), as result of their research, claimed that any other structure not contemplated 
in the geometrical range studied by Zarghamee and Heger should be analyzed by a different approach, since unconservative results could be obtained otherwise.

Therefore, to summarize the results of all the previous investigations, in order to accurately predict the ultimate buckling failure of these structures, the combined effect of different sources of nonlinearities should be included in the analysis, since these structures are highly sensitive to any deviation of their ideal conditions (Mekjavić, 2011).

\subsection{ACI 372R-13 design method}

ACI372R-13 provides recommendations for the design and construction of wrapped, circular, prestressed concrete structures. Since these elements are commonly used for liquid or bulk storage, the design of a roof is usually required. To this effect, ACI recommends the use of concrete domes as a suitable roofing structure.

According to ACI, buckling is the dominant failure mode of this type of structures and thus their design should be based on a rational analysis of their buckling capacity leading to the minimum thickness required to resist such effect. The latest version of the code has adopted the equation by Zarghamee and Heger (1983) for the design of these structures, the only difference being the definition of the applied pressure. Zarghamee defined this pressure as a simple critical load $\left(P_{o}\right)$ that only included the dead and live load effects which was compared with the total factored load, as follows:

$$
\text { 1.4DL +1.7LL } \leq P_{o}
$$

While ACI replaces $P_{o}$ with two components: $\mathrm{Pu}$ and $\mathrm{Ev}$. The first parameter, similar to Zarghamee's study represents the factored dead and live loads; while, the second one is a new parameter representing the inclusion of the vertical component of earthquake. In this manner, ACI specifies the following load combinations to be used for the design of this type of structures: 
Table 1. Load combinations for design of spherical shells as per ACI372-13

\begin{tabular}{|c|}
\hline Load condition $1: \mathrm{U}_{1}=1.4 \mathrm{D}$ \\
\hline $\begin{array}{l}\text { Where: } \\
\mathrm{P}_{\mathrm{u}}=1.4 \mathrm{D}, \mathrm{lb} / \mathrm{ft}^{2}(\mathrm{kPa}) \\
\beta_{\mathrm{c}}=0.44 \\
\mathrm{E}_{\mathrm{v}}=0\end{array}$ \\
\hline Load condition 2: U2 $=1.2 \mathrm{D}+1.6 \mathrm{~L}$ \\
\hline $\begin{array}{l}\text { Where: } \\
\mathrm{P}_{\mathrm{u}}=1.2 \mathrm{D}+1.6 \mathrm{~L}, \mathrm{lb} / \mathrm{ft}^{2}(\mathrm{kPa}) \\
\beta_{\mathrm{c}}=0.44+0.003 \mathrm{~L} \text { but not greater than } 0.53 \\
\beta_{\mathrm{c}}=0.44+0.063 \mathrm{~L} \text { but not greater than } 0.53 \text { when units are in } \mathrm{kPa} \\
\mathrm{E}_{\mathrm{v}}=0\end{array}$ \\
\hline Load condition $3: \mathrm{U} 3=1.2 \mathrm{D}+0.2 \mathrm{~S}+\mathrm{E}_{\mathrm{v}}$ \\
\hline $\begin{array}{l}\text { Where: } \\
\mathrm{P}_{\mathrm{u}}=1.2 \mathrm{D}+0.2 \mathrm{~S}, \mathrm{lb} / \mathrm{ft}^{2}(\mathrm{kPa}) \\
\mathrm{B}_{\mathrm{c}}=0.44+0.000375 \mathrm{~S} \\
\mathrm{~B}_{\mathrm{c}}=0.44+0.00783 \mathrm{~S} \text { when units are in } \mathrm{kPa} \\
\mathrm{S} \text { : Uniformly distributed snow load in accordance with ASCE } 7(2010), 1 \mathrm{~b} / \mathrm{ft}^{2}(\mathrm{kPa}) \\
\mathrm{E}_{\mathrm{v}} \text { : Vertical earthquake component to be calculated assuming an importance factor of } 1.0 \text {, } \\
\text { a modification factor of } 1.0 \text { and a vertical acceleration equal to } 2 / 3 \mathrm{~S}_{\mathrm{DS}} \text { of the lateral } \\
\text { mapped acceleration from ASCE } 7(2010) \text { or } \mathrm{A}_{\mathrm{v}} \text { if site specific vertical acceleration is to } \\
\text { be used. }\end{array}$ \\
\hline
\end{tabular}

As can be noticed in Table 1, the code only considers the effect of gravity loads and the vertical component of earthquake and no mention whatsoever is made of lateral loads or the horizontal component of earthquake.

The ACI equations, as well as the formulation suggested by Zarghamee (1983) are based on the elastic theory of dome shell stability, including some adjustments to the formulation to take into account the nonlinearities of a reinforced concreted shallow spherical cap, resulting in the following equation for the required minimum thickness:

$$
h_{d}=r_{d} \sqrt{\frac{1.5}{\varnothing B_{i} E_{c}}\left(\frac{P_{u}}{B_{c}}+E_{v}\right)} \text { in }
$$




$$
h_{d}=r_{d} \sqrt{\frac{1.5 \times 10^{-3}}{\emptyset B_{i} E_{c}}\left(\frac{P_{u}}{B_{c}}+E_{v}\right)} \mathrm{mm}
$$

Where, the terms of the formulation, can be determined by the following equations:

$$
\begin{gathered}
\phi=0.6 \\
B_{i}=\left(\frac{r_{d}}{r_{i}}\right)^{2} \\
E c=57000 \sqrt{f^{\prime} c} p s i \\
E c=4730 \sqrt{f^{\prime} c} M P a
\end{gathered}
$$

The Code also specifies the following criteria to be met:

- The minimum shell thickness is 3 in $(75 \mathrm{~mm})$.

- $\quad$ The minimum 28-day compressive strength of concrete (f'c) is 4000 psi (28MPa).

- Normal-weight aggregates are to be used.

The Code does not limit the geometry of the dome, however it provides the following recommendations for design:

- The ideal rise-to-span ratio is considered as 1:10, however a range between 1:12 and 1:8 is commonly accepted in the industry.

- The thickness of domes should not be less than 3 in $(75 \mathrm{~mm})$ for monolithic concrete and shotcrete, $4 \mathrm{in} .(100 \mathrm{~mm})$ for precast concrete, and $2 \frac{1}{2} \mathrm{in}$. $(65 \mathrm{~mm})$ for the other shell of a ribbed dome.

- In edge regions of thin domes and throughout domes over 5in. (130mm) thick, nonprestressed reinforcement should be placed in two layers.

- The minimum ratio of non-prestressed reinforcement area to concrete area in the dome 
ring should be 0.0025 for cast-in-place dome rings.

The design should meet the requirements indicated in this Code, as well as the specifications of ACI 318 and ACI 350. 


\section{CHAPTER 3}

\section{FINITE ELEMENT MODELING}

\subsection{Introduction}

As mentioned in previous chapters, different analytical formulations have been proposed over several years to analyze the behavior of spherical shells; however, in all instances, some assumptions had to be made to simplify the formulation, due to the complexity of the subject.

Therefore, even if these formulations provide a solution for the buckling problem, they only represent general scenarios; for instance, the classical buckling formulation provides a linear elastic solution to a bifurcation buckling problem when the structure is under the effect of uniform pressure; while, in the other case, the equation proposed by Zhargamee (1983) also provides a solution when the structure is subjected to uniform pressure but this time yielding the nonlinear snap-through buckling solution of the problem.

As seen and mentioned before, both equations provide a general solution; however, when both the vertical and horizontal load components are applied simultaneously, as is usually the case during any seismic event, such condition is not clearly contemplated in neither of the formulations. Therefore, in this case it is more practical to use a numerical technique such as Finite Element Method (FEM), which is able to provide accurate solutions to complex problems in little time; allowing at the same time multiple types of analysis, the

inclusion of different parameters; and consequently a deeper understanding of the nature of this challenging problem.

However, the effectiveness of FEM will rely on a thorough understanding of the theories proposed for this type of structures as well as the procedures followed by the method; therefore, initially in this chapter a brief discussion of the basic equations that led to the development of the classical formulation is presented, including also the adjustments 
made for the development of the nonlinear analysis.

Finally, the chapter provides a brief discussion on the considerations made throughout the analysis to ensure accurate results; such as the program selection, type of elements, mesh size and tools used. Moreover, additional and specific information is also given in the next chapters.

\subsection{Finite element method}

The Finite Element Methods are numerical procedures developed in the early 1960s in order to give an approximate solution to complex mathematical problems. However, it has only been in the last few decades that with the development of the Finite Element Analysis (FEA), the method has started to reach its potential.

This innovation was followed by the development of engineering software coded with FE algorithms; offering the possibility of giving solutions to problems of different natures, such as linear and/or nonlinear structural, mechanical, electrical, thermal, etc. by solving partial differential equations in a relatively short amount of time.

The technique consists of dividing the model into smaller and simpler elements and connecting them by their nodes. Based on the type of element, an algebraic equation known as the shape function will be assigned to it in order to interpolate the solution between its nodes. After some mathematical procedures elements are assembled together into one large system of equations that represents the entire structure. This is the point where the use of FEA software is necessary, since the solution of the system cannot be achieved manually.

Gupta (2008), performed an experimental analysis in order to verify the proposed FEA. Based to the results obtained from the load-compression curves and the deformed shape observed in Figure 15, the FEA showed a good agreement with the experimental results, allowing with this to carry out further analysis on more challenging problems. 


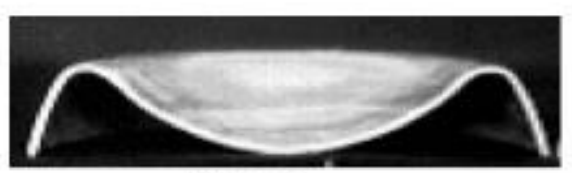

(a) Actual

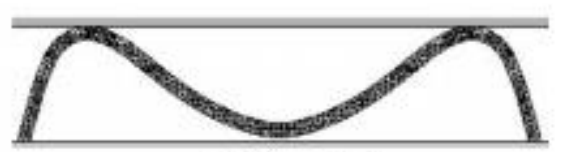

(b) Computed

Figure 15. Comparison of actual and FEA predicted failure of spherical shell

Therefore, as can be observed, this method offers numerous advantages; however, it should be clarified that the generated solutions are not exact, but approximate; and that the effectiveness of this tool will only depend on the user and the decisions made throughout the process.

In this research the FEA software ABAQUS was selected to perform the numerical analysis. This is a computer-aided engineering tool developed and supported by SIMULIA® that has proven over the years to be versatile and reliable in idealizing the problems of different disciplines in a clear and concrete way. The software has become one of the leading FEA programs not only in the Civil Engineering field but in the entire engineering industry.

For the analysis, two core products of the software will be used: ABAQUS/CAE and ABAQUS/Standard; the first one is the modeling environment for all the pre and post processing needs, from the definition of the material to the visualization of the results; while the second one, corresponds to the analysis and solution of the problem. For the purpose of this study, ABAQUS/standard was selected instead of ABAQUS/explicit, since this offers an ideal tool to solve general problems where no dynamic circumstances are considered.

For the purpose of analysis of this specific problem, several analysis steps need to be defined; the first one called the initial step corresponds to the initial conditions that need to be assigned at the very beginning of the analysis, such as boundary conditions and interactions.

Subsequently, more steps should be added in accordance to what is to be determined, 
these are called analysis steps, and could be divided into two types: general analysis and linear perturbation steps. A general step, Standard or Explicit, will depend on the type of analysis chosen; however, despite the procedure, it is in this step that the effects of the nonlinearities should be included. While, on the other hand, the linear perturbation is a substep that analyzes the linear response of the structure based on the final state of the last general step performed.

In this investigation, two types of general steps namely General Static and Static Riks and two types of linear perturbations namely Buckle and Frequency sub-steps are used. In the following chapters, a more detailed explanation of each of these will be provided.

\subsection{Element selection}

In order to initiate the model and in accordance with the requirements of performing a FE analysis, ABAQUS requires the selection of element types to be made during the first step. A careful selection of the type of elements is highly important in achieving a precise prediction of the behavior of the structure under consideration.

This program in particular offers a long list of different elements to select from, as shown in Figure 16; however, the selection will always depend on the type of structure to be modelled and its expected behavior. In this case, among all the options, the element type S4R is selected, since it is considered to be the most convenient one for representing the behavior of a typical dome structure. This is a quadrilateral four-node element with six degrees of freedom per node (translation in the nodal $\mathrm{x}, \mathrm{y}$ and $\mathrm{z}$ directions and rotations about the nodal $\mathrm{x}, \mathrm{y}$ and $\mathrm{z}$ axes), and with reduced integration and large-strain formulation capabilities. 


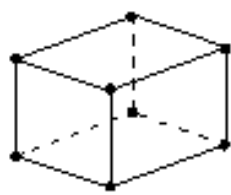

Continuum (solid) elements

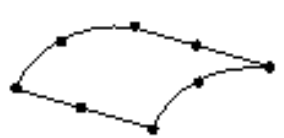

Membrane elements

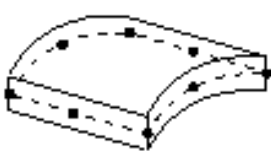

Shell elements

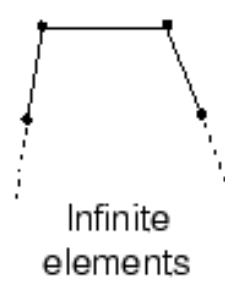

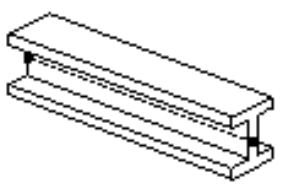

Beam elements
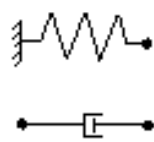

Springs and dashpots

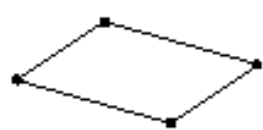

Rigid elements

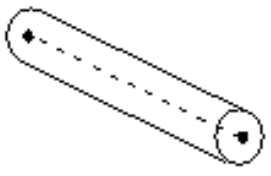

Truss elements

Figure 16. ABAQUS element families

The classical theory of buckling proposes the use of thin elements and therefore neglects the contribution of second order transverse normal shear strain and stress terms. As mentioned before, such assumption does not yield accurate results. It was further discussed that one of the reasons for the large discrepancy between measured and predicted results was this and other simplifying assumptions made in deriving the final formulation. Nowadays, with the development of powerful FE computer programs, such as ABAQUS, those simplifications are not needed anymore, opening a possibility for obtaining more precise results in little time.

Therefore, the selection of this type of element is also encouraged by the fact that it considers the thick shell theory, accounting then for the effects of transverse shear, largestrains, and changes in element thickness with deformation.

Another main reason promoting the selection of this type of element was the need for using an element allowing the embedment of rebar. ABAQUS emphasizes that rebars cannot be used with triangular shells or membranes; therefore, a quadrilateral shell element had to be used.

As for the case of the type of integration to be used for the calculation of the stiffness 
matrix, the analysis is simplified to a reduced integration problem with a single integration point per element, in contrast to a typical full integration of four points. Clearly, the more points are used, the more accurate the calculation will be; however, when weighting the cost of computational time involved and the minor effects that the simplification has in the accuracy of the results, the reduced integration is found suitable for the analysis. The geometry and characteristics of this element are shown in Figure 17.

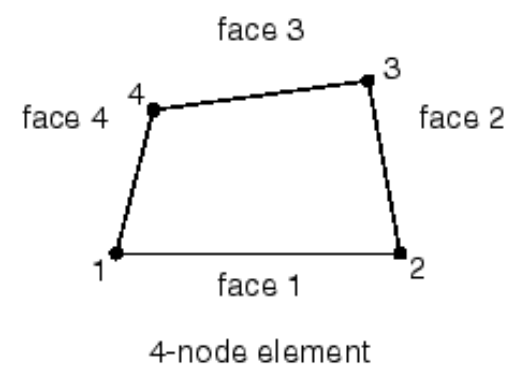

( a)

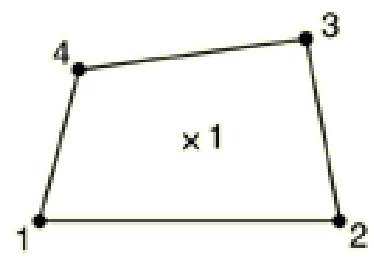

4-node reduced integration element

( b)

Figure 17. Finite element geometry; (a) Node ordering, (b) Number of integration points (Adapted from ABAQUS manual)

\subsection{Mesh size}

Generally, a smaller mesh size or equally an increase in the number of elements, leads to more accurate results. However, this usually increases the running time of the model. In addition, the use of many degrees of freedom, may not necessarily lead to a considerable change in final results, where there is no justifiable reason for doing so. Therefore, a convergence analysis should be carried out, in order to find a suitable mesh size for each model and guarantee accurate results in a convenient amount of time.

The convergence study is performed by the determination of the buckling pressure of the structure using four different mesh sizes per model and comparing the FE results with the analytical solution. The percentage of error is then estimated as: 


$$
\mathrm{E}(\%)=\frac{\left(p_{E X}\right)-\left(p_{F E}\right)}{\left(p_{E X}\right)} \times 100
$$

Where, $\mathrm{P}_{\mathrm{FE}}$ represents the buckling pressure obtained through $\mathrm{FE}$ analysis and $\mathrm{P}_{\mathrm{EX}}$ is the exact analytical solution based on the formulation proposed by Zhargamee and Heger (1983) currently adopted by ACI. The optimum mesh size is then introduced when the difference between the two consecutive error values could be considered negligible. Figure $18(\mathrm{a}-\mathrm{n})$ is a plot of the resulting percentage error versus the corresponding mesh size obtained for each of the 14 models used in this research. The model properties will be later defined in chapter 4.

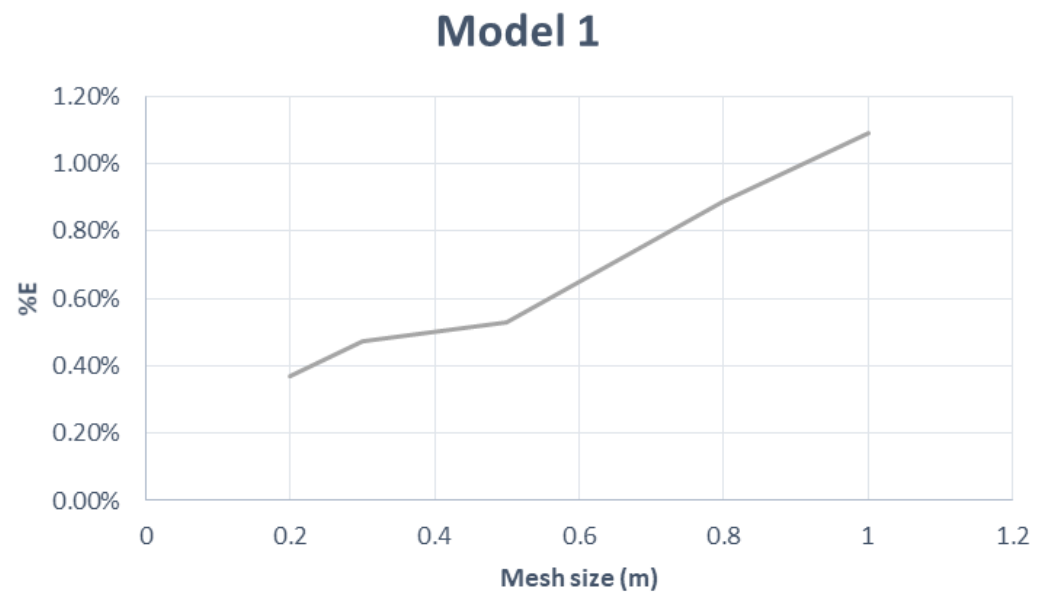

(a)

Figure 18. Inaccuracy of FE solution as a function of mesh size 


\section{Model 2}

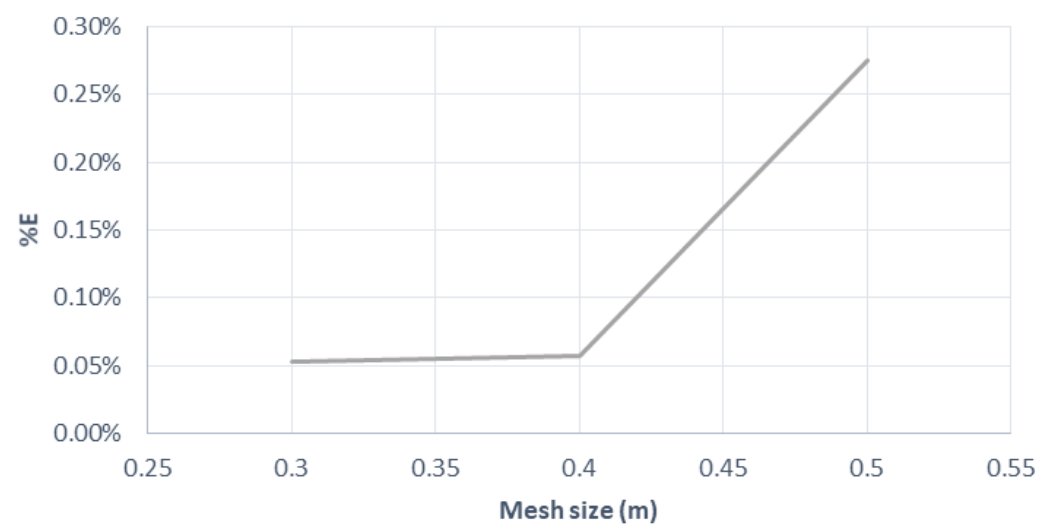

(b)

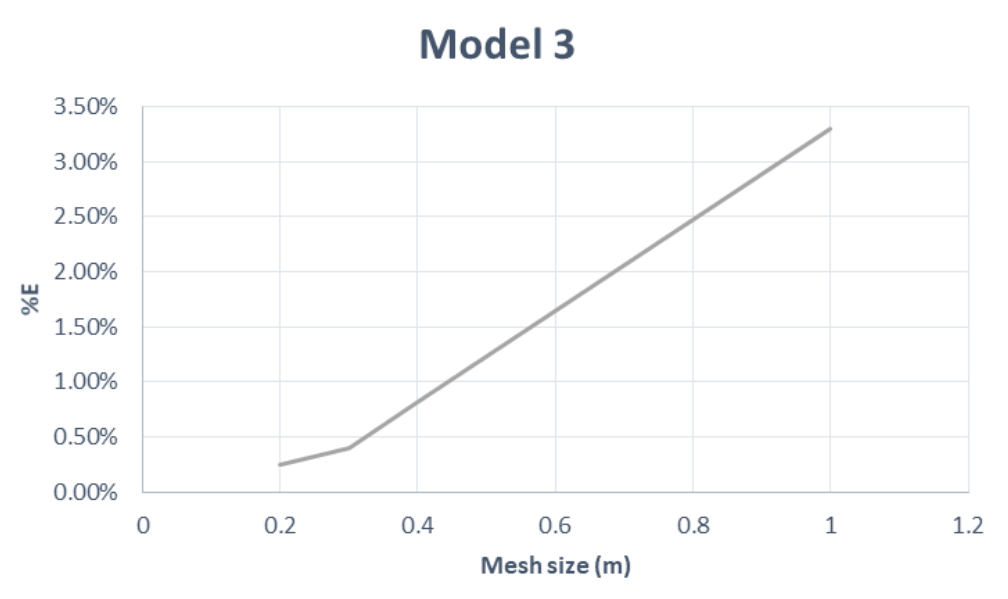

(c)

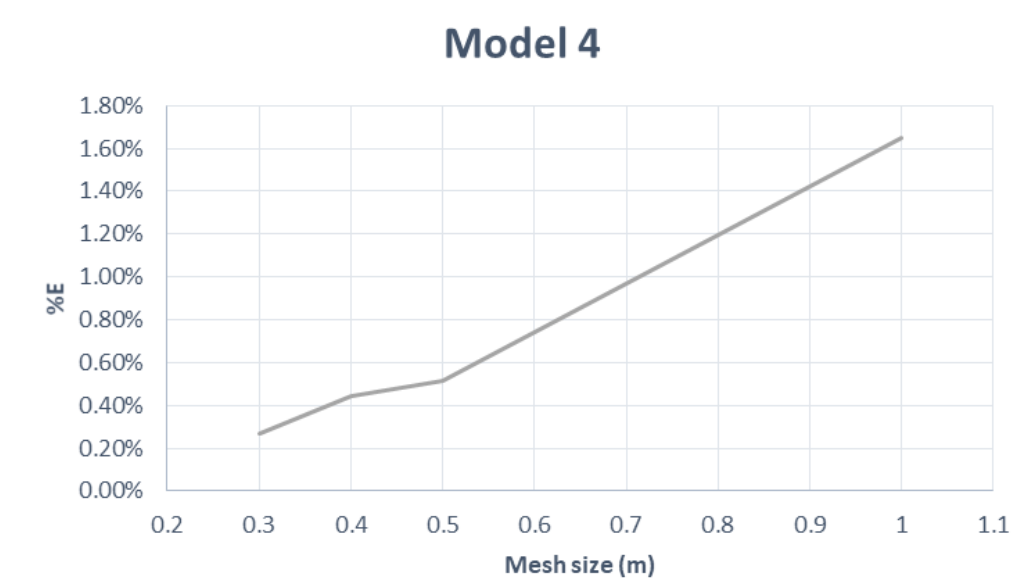

(d)

Figure 18. Inaccuracy of FE solution as a function of mesh size, Continued 


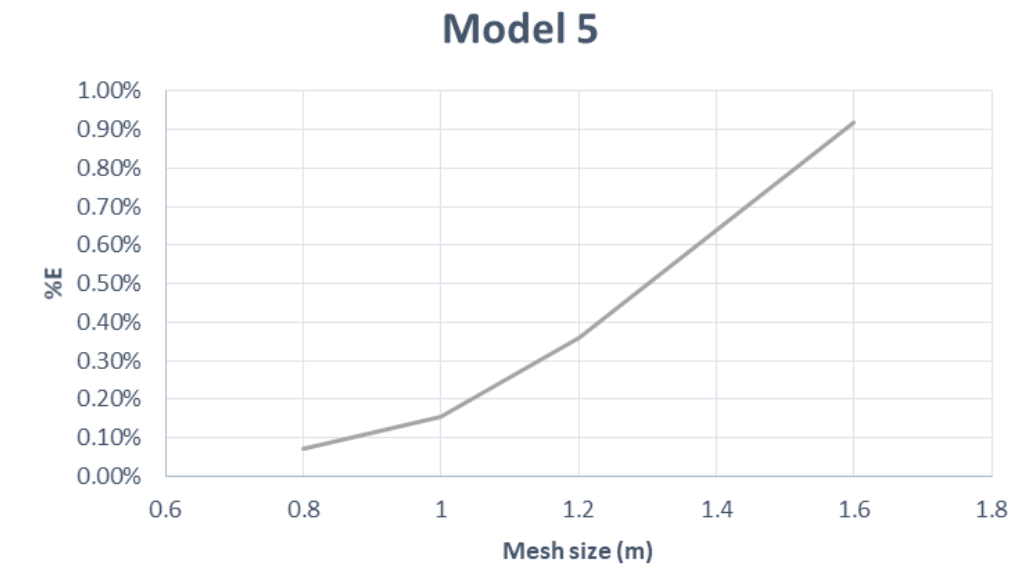

(e)

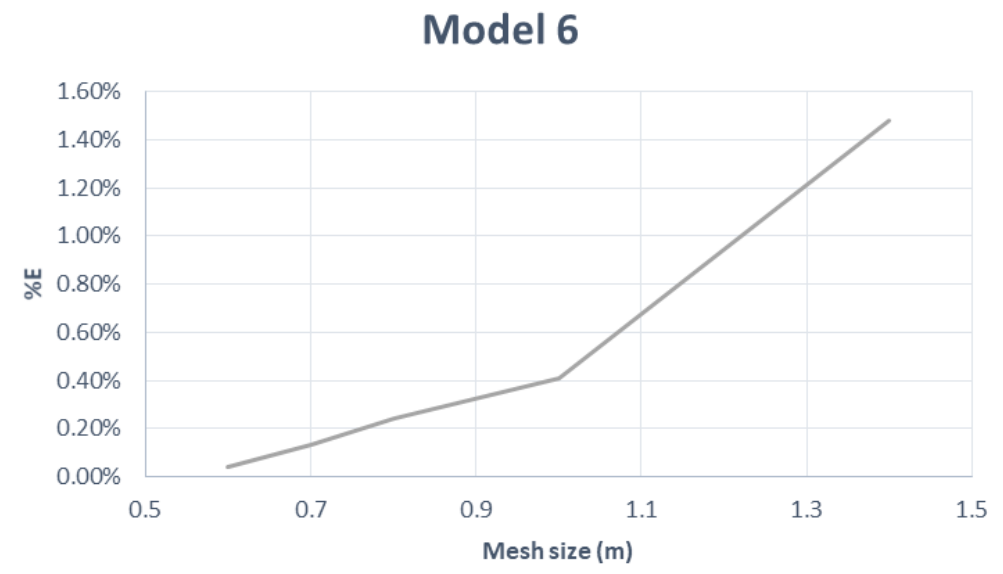

(f)

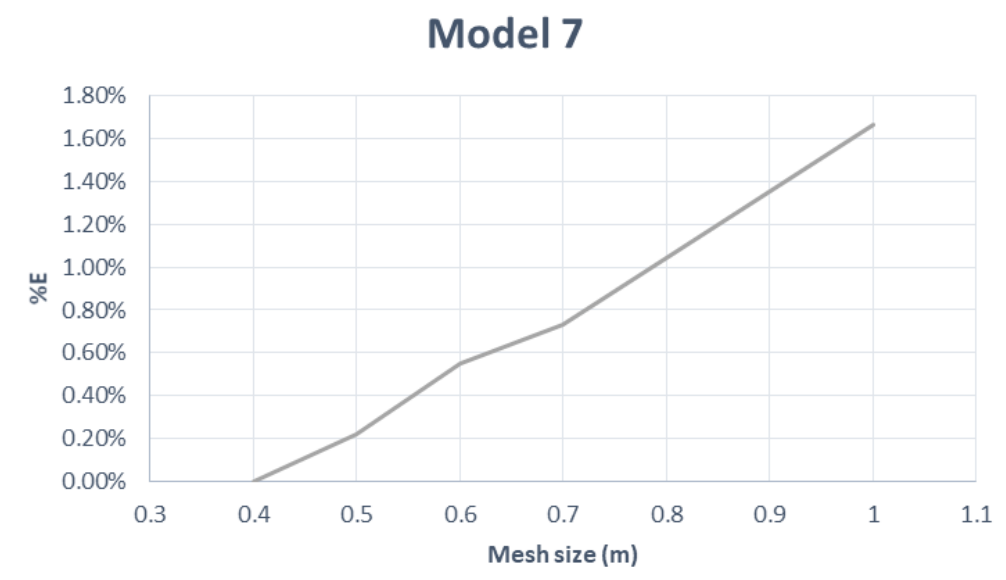

$(\mathrm{g})$

Figure 18. Inaccuracy of FE solution as a function of mesh size, Continued 


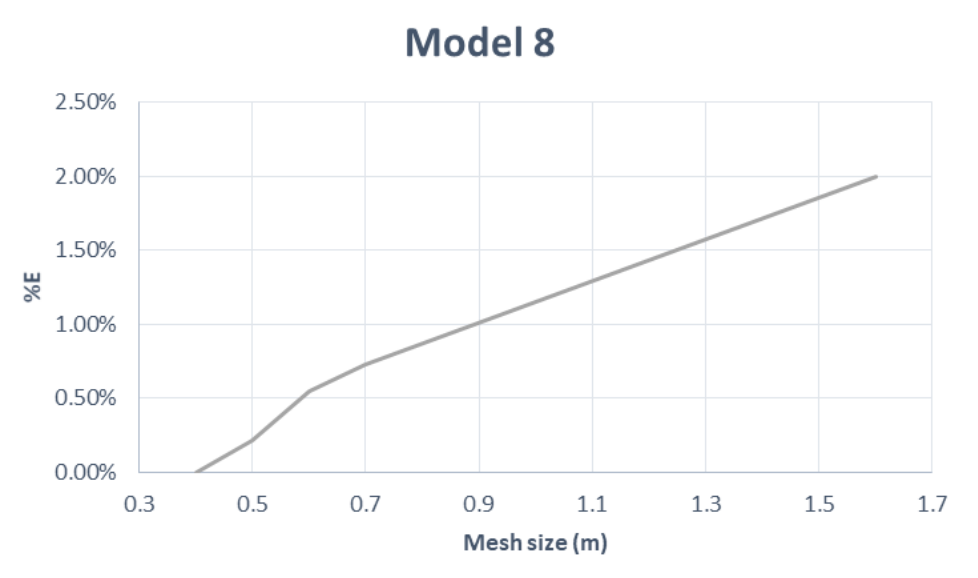

(h)

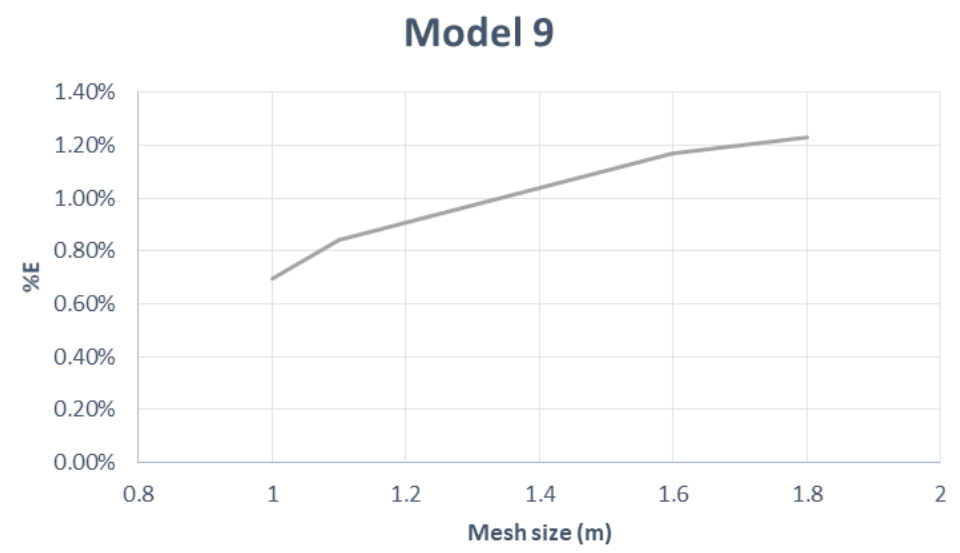

(i)

Model 10

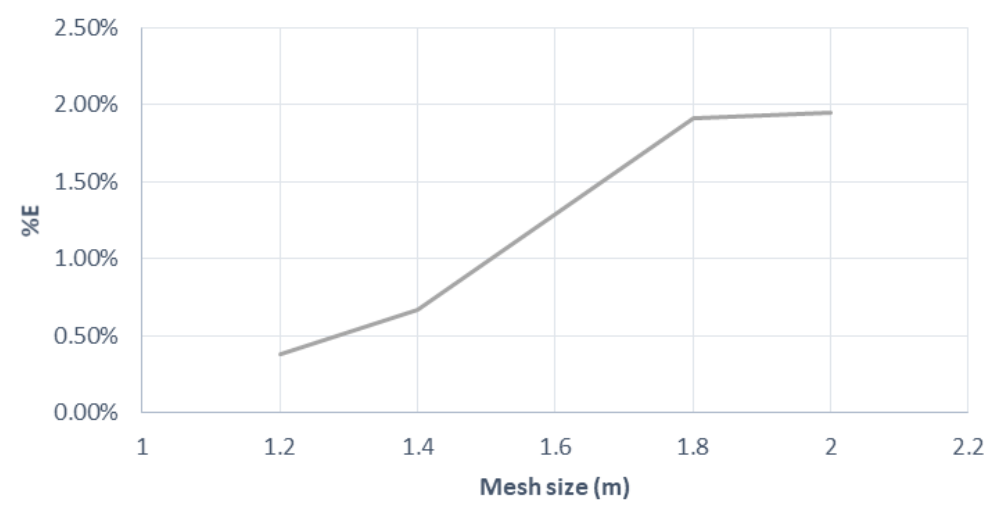

(j)

Figure 18. Inaccuracy of FE solution as a function of mesh size, Continued 


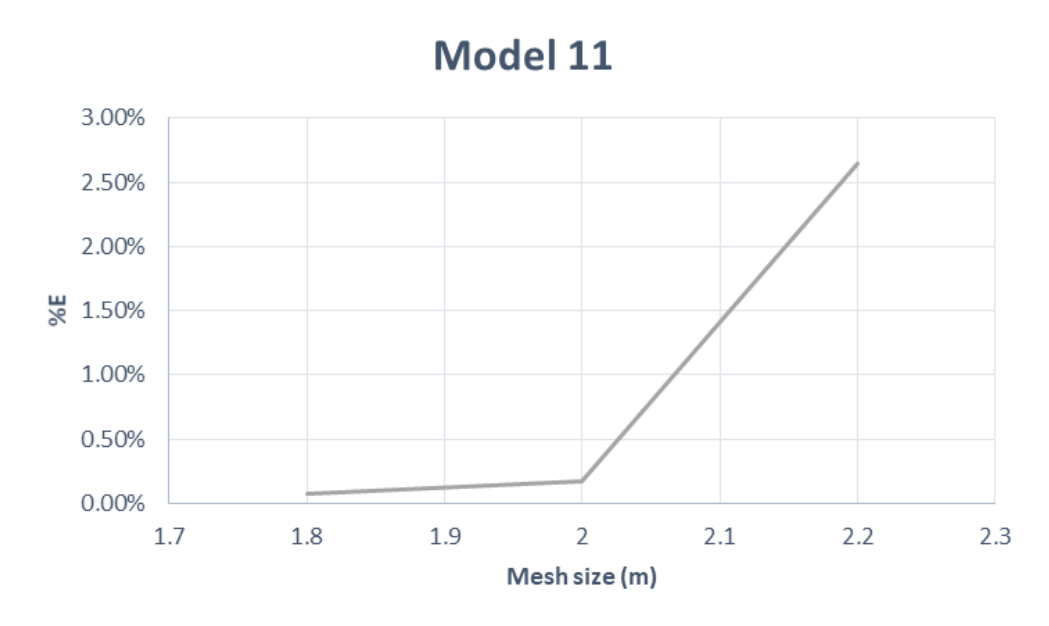

(k)

Model 12

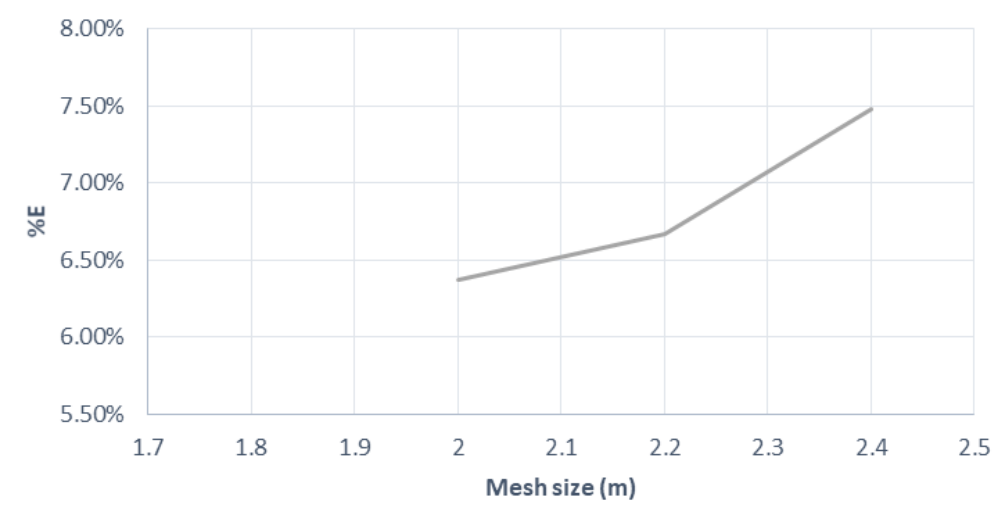

(1)

Model 13

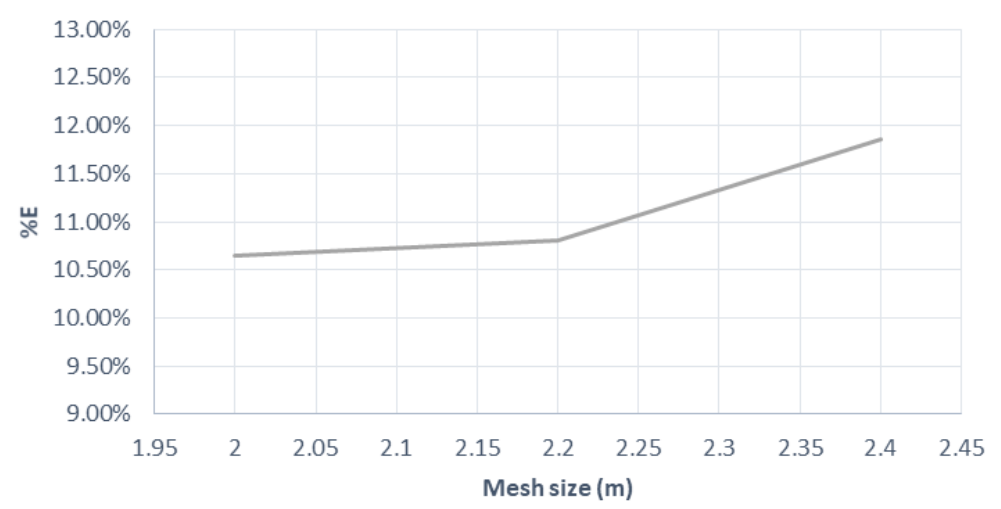

(m)

Figure 18. Inaccuracy of FE solution as a function of mesh size, Continued 


\section{Model 14}

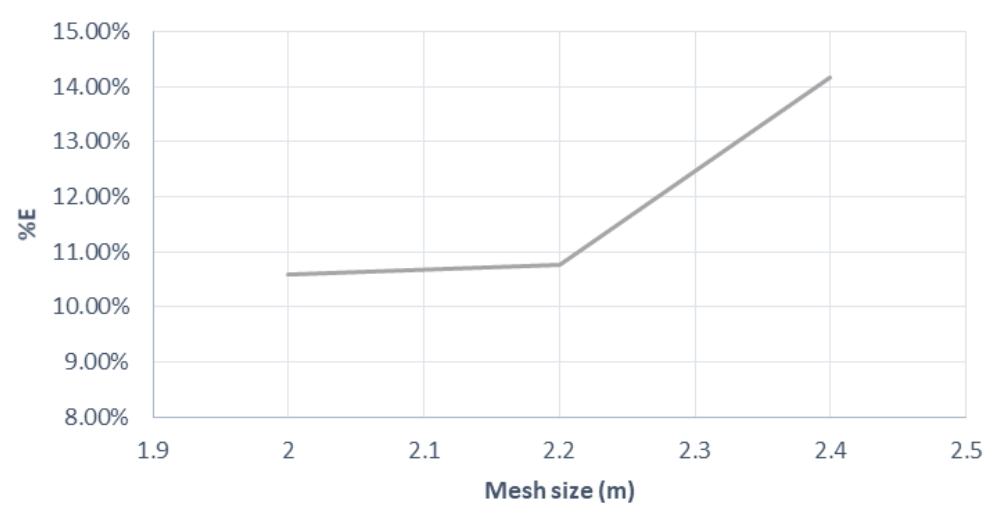

(n)

Figure 18. Inaccuracy of FE solution as a function of mesh size, Continued

From the above study and taking into account the running time of each model, the following mesh schemes are adopted for the analysis as optimum mesh sizes returning precise results in a reasonable time frame:

Table 2. Mesh size selection for each model

\begin{tabular}{|c|c|c|c|}
\hline Model & $\begin{array}{c}\text { Area of the spherical cap } \\
\left(\mathrm{m}^{2}\right)\end{array}$ & Seed size $(\mathrm{m})$ & $\begin{array}{c}\text { Error } \\
(\%)\end{array}$ \\
\hline 1 & 188 & 0.30 & $0.47 \%$ \\
\hline 2 & 750 & 0.40 & $0.06 \%$ \\
\hline 3 & 187 & 0.30 & $0.40 \%$ \\
\hline 4 & 747 & 0.40 & $0.44 \%$ \\
\hline 5 & 1690 & 0.80 & $0.07 \%$ \\
\hline 6 & 2295 & 0.80 & $0.24 \%$ \\
\hline 7 & 762 & 0.40 & $0.00 \%$ \\
\hline 8 & 1710 & 0.80 & $0.41 \%$ \\
\hline 9 & 2327 & 1.00 & $0.69 \%$ \\
\hline 10 & 3109 & 1.40 & $0.67 \%$ \\
\hline 11 & 4863 & 1.80 & $0.07 \%$ \\
\hline 12 & 6995 & 2.00 & $6.38 \%$ \\
\hline 13 & 9528 & 2.00 & $10.65 \%$ \\
\hline 14 & 12433 & 2.00 & $10.60 \%$ \\
\hline
\end{tabular}


In most of the cases the selected mesh produces an error below 1\%; however, it is noted that for the last three models an error percentage of $6 \%$ to $10 \%$ is obtained. This is to be expected as the last three models have two layers of rebar resulting in a substantial increase in the buckling capacity from FE analysis whereas such consideration is not taken into account in the analytical formulation.

\subsection{Summary}

Through this chapter, some fundamental information needed to perform an accurate finite element analysis was discussed; from method definition to mesh size selection.

Initially, the finite element software to perform the analysis was selected, adopting ABAQUS as the most suitable program for this investigation; considering that this is a trustworthy software and a leader in the area that provides necessary engineering tools to carry out an effective analysis of this kind of problem.

Subsequently, regarding the type of element, a quadrilateral four-node element; S4R with six degrees of freedom per node and a reduced integration rule was chosen. The element is deemed suitable for the idealization of the problem as it is a general purpose shell element capable of incorporating into the formulation the transverse shear deformation.

With respect to the size of the mesh, the optimum size was reached for each model after several attempts and through a trial and error approach in which the goal was to achieve accurate results in a short period of time. However, since each model had a different geometric configuration, a particular mesh size was assigned to each; which is then to be used for both linear and nonlinear types of analysis.

In addition, a summary of different types of analysis to be employed during the present research was mentioned which will be further explained in the following chapters, as they are applied. 


\section{CHAPTER 4}

\section{BUCKLING OF SPHERICAL SHELLS}

\subsection{Introduction}

In this chapter, there are two objectives to be achieved. First is the verification of the proposed finite element technique which will be carried out by comparing the results obtained from this method with the corresponding analytical formulation. While, the second objective aims at analyzing the buckling failure of spherical shells under the influence of different parameters, such as geometric configuration, imperfection, base fixity, and rebar arrangement. To provide a response to this effect, different variations of these parameters are used with the intention of later contrasting the results obtained.

However, before directly addressing these two objectives, it is essential to first define the models that will be considered throughout this chapter. Model properties including the geometric configurations, material properties and details of reinforcement are described in detail in the following sections.

Once the basis is set, the chapter is divided into two phases: first, being the linear analysis step, which deals with the bifurcation buckling of the structure. Through this phase, the obtained FE results are compared with those calculated from the classical buckling equation. Once the modeling accuracy is verified under linear conditions, the analysis proceeds to the second phase, being buckling response under nonlinear conditions, where the purpose is to determine the snap-through buckling capacity considering imperfection effects both numerically and analytically. This is followed by a comparison of results to verify the accuracy of the proposed nonlinear model.

Additionally, in this chapter, further description of the procedures used to determine linear and nonlinear buckling phenomena is provided. 


\subsection{Models properties}

In this study, a wide range of spherical shell configurations is considered with the intention of analyzing the influence of geometry on the buckling behavior. To this effect, 14 different geometric arrangements are used, obtained by varying the angle, thickness and radius of the structure.

According to a survey conducted by Zhargamee and Heger (1983), most common Span-to-Rise ratios used for the roof of concrete water tanks ranged from 8 to 14; while, with respect to thickness, most of the structures were quite thin with their thickness being in most cases about 3" (76 mm) which is the minimum specified by ACI.

Therefore, in an effort to select practical and close to reality modeling case scenarios, the above mentioned values are taken into consideration, but also expanded to a larger range; as was also carried out in the study by Zolqadr, E (2017).

The summary of different geometric configurations employed is described in accordance with Figure 19, in Table 3:

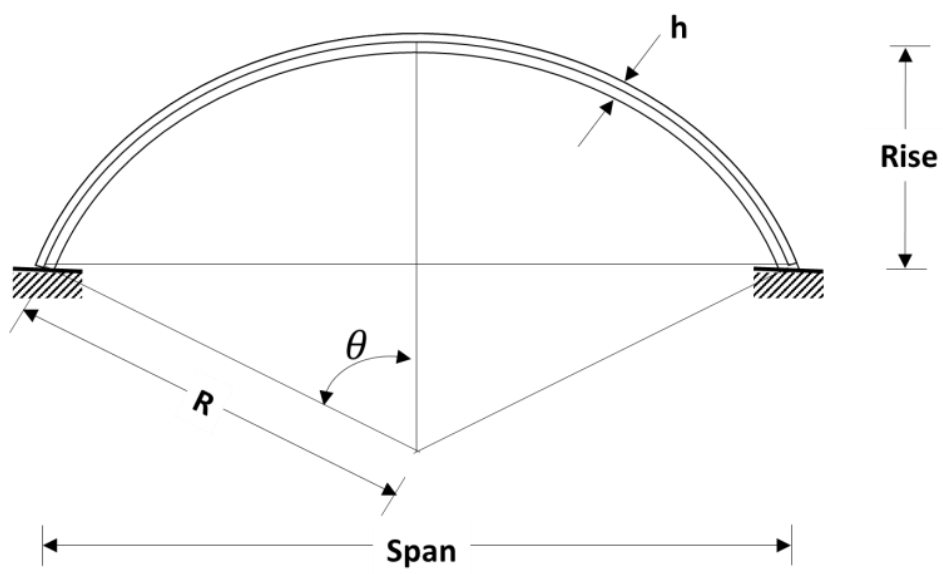

Figure 19. Geometry of spherical shell 
Table 3. Geometry of the models

\begin{tabular}{|c|c|c|c|c|c|c|c|}
\hline Model & $\begin{array}{c}\mathrm{R} \\
(\mathrm{m})\end{array}$ & $\begin{array}{c}\text { Rise } \\
(\mathrm{m})\end{array}$ & $\begin{array}{c}\text { Span } \\
(\mathrm{m})\end{array}$ & $\begin{array}{c}\theta \\
(\mathrm{o})\end{array}$ & $\begin{array}{c}\mathrm{h} \\
(\mathrm{mm})\end{array}$ & $\mathrm{R} / \mathrm{h}$ & Span to rise \\
\hline 1 & 27.22 & 1.09 & 15.3 & 16 & 76 & 358 & 14 \\
2 & 54.43 & 2.18 & 30.6 & 16 & 76 & 716 & 14 \\
3 & 25.35 & 1.17 & 15.2 & 17 & 76 & 334 & 13 \\
4 & 50.7 & 2.34 & 30.5 & 17 & 76 & 667 & 13 \\
5 & 76.05 & 3.52 & 45.8 & 17 & 102 & 746 & 13 \\
6 & 88.73 & 4.1 & 53.4 & 17 & 102 & 870 & 13 \\
7 & 39.62 & 3.05 & 30.5 & 23 & 102 & 388 & 10 \\
8 & 59.44 & 4.57 & 45.8 & 23 & 89 & 668 & 10 \\
9 & 69.34 & 5.33 & 53.4 & 23 & 95 & 730 & 10 \\
10 & 64.77 & 7.62 & 61.0 & 28 & 95 & 682 & 8 \\
11 & 80.96 & 9.53 & 76.3 & 28 & 114 & 710 & 8 \\
12 & 97.16 & 11.43 & 91.6 & 28 & 146 & 665 & 8 \\
13 & 113.35 & 13.34 & 106.8 & 28 & 190 & 597 & 8 \\
14 & 129.54 & 15.24 & 122.1 & 28 & 235 & 551 & 8 \\
\hline
\end{tabular}

With regard to the dome base boundary condition, a fixed connection is assumed at the supports. However, the influence of this type of connection will be further analyzed later under section 4.5.5.

Finally, the properties of concrete and steel materials used in modeling the shells are summarized in Table 4.

Table 4. Material properties

\begin{tabular}{|c|c|c|}
\hline Material & Concrete & Steel \\
\hline Density $\left(\mathrm{kg} / \mathrm{m}^{3}\right)$ & 2400 & 7850 \\
\hline $\mathrm{f} ' \mathrm{MPa})$ & 28 & - \\
\hline $\mathrm{E}(\mathrm{MPa})$ & 25029 & 200000 \\
\hline $\boldsymbol{v}$ & 0.17 & 0.3 \\
\hline
\end{tabular}

The finite element idealization of reinforced concrete shells will be explained under section 4.3 . 


\subsection{Finite element modelling of reinforced concrete shells}

Since this investigation intends to analyze the behavior of reinforced concrete shells, the use of a composite section is mandatory. ABAQUS provides a useful tool that allows the definition of one or multiple layers of rebar in shell elements.

In order to create these layers, first concrete and steel material specifications are defined in accordance with the information given in Table 4. Subsequently, a local coordinate system is defined and assigned to the element; by which the direction of rebar in the element's cross-section can be identified. However, this rebar orientation system is completely independent from the global orientation system used for the structure. For this purpose, a cylindrical coordinate system is used since a typical reinforcement arrangement for this type of structures, as also specified by ACI, is made of layers of reinforcement aligned circumferentially and radially as shown in

Figure 20.

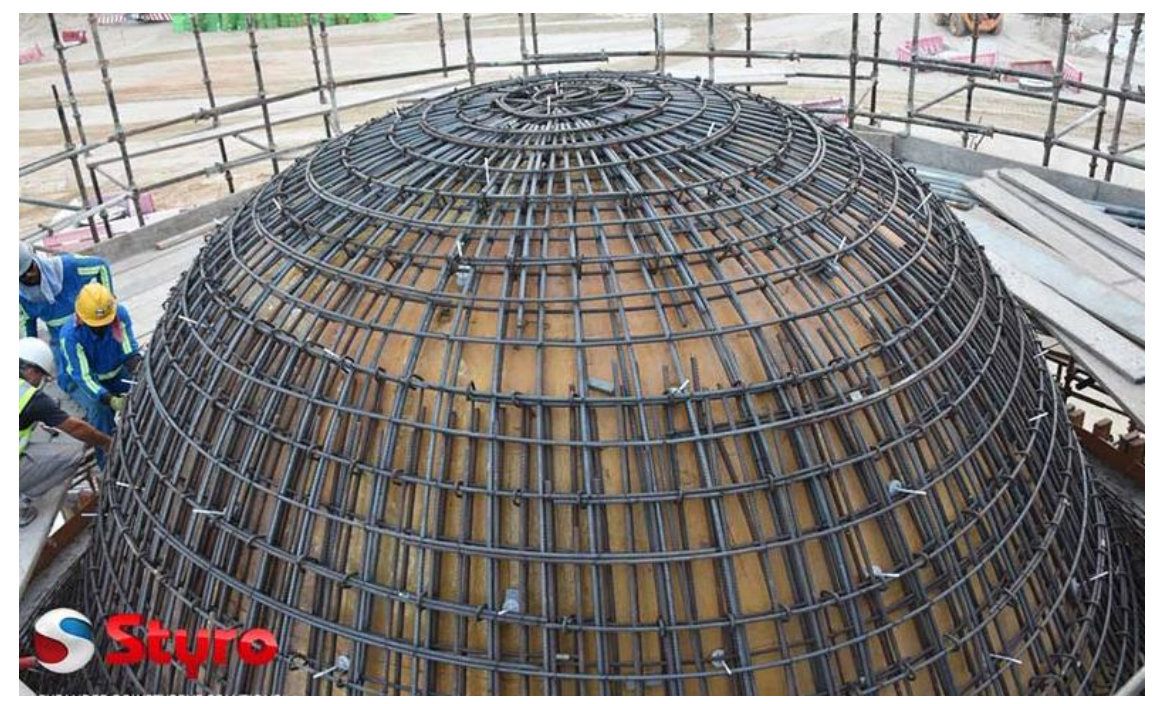

Figure 20. Spherical shell reinforcement (Adapted from https://www.styrouae.com)

Even though, ABAQUS allows for the creation of each one of these layers, 
individually, the implementation of both layers simultaneously is not readily possible in ABAQUS/CAE. As a result, the introduction of these layers is done manually by modifying the keyword in FE model, as shown in Figure 21 for model 1.

\section{Edit keywords, Model: Model-1}

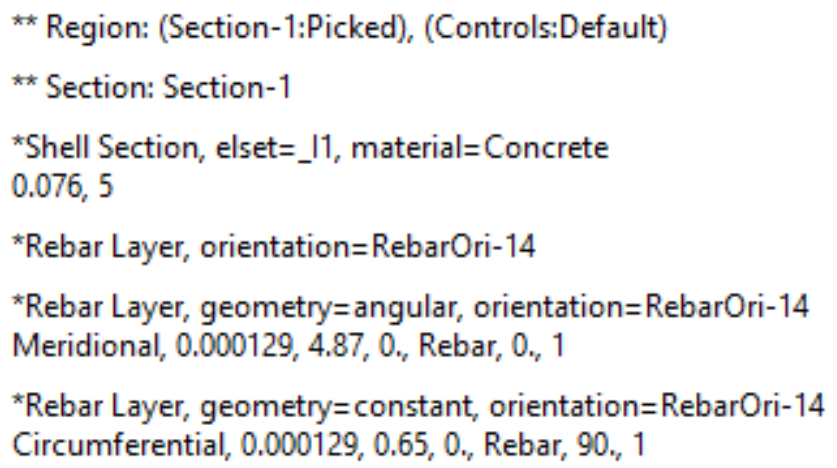

\section{Figure 21. Shell reinforcement (ABAQUS keyword modification)}

As shown in Figure 21, the new command lines to define multiple layers of reinforcement are described as "Rebar Layer"; where; the first parameter corresponds to the geometry, either constant or angular, depending on the direction of the rebar described in Figure 22 and Figure 23; the second parameter is the orientation, which is set equal to the name of the rebar orientation system previously defined. Subsequently, in the data lines the rest of the rebar information is defined in the following order: name of the layer, area of reinforcement, spacing (length or degrees), position, material, orientation and isoparametric direction. This data line is repeated as many times as layers as necessary.

In this manner, two layers were created, a "Meridional" layer and a "Circumferential" layer; the first one is considered to be radial with an orientation angle equal to zero, while the second one is circumferential with a $90^{\circ}$ orientation, as shown in Figure 22 and Figure 23, respectively. Further information on the area and spacing of the reinforcement is provided in Table 5. 


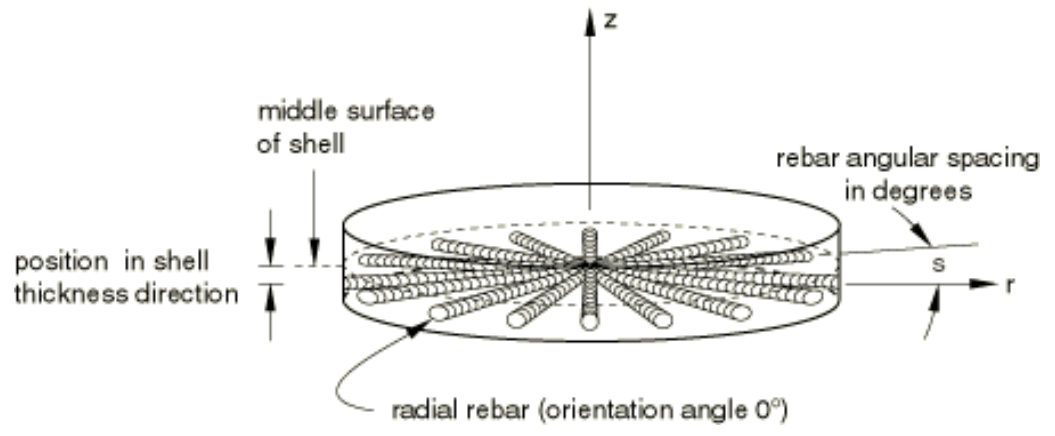

Figure 22. Radial rebars in axisymmetric shell elements (Adapted from ABAQUS manual)

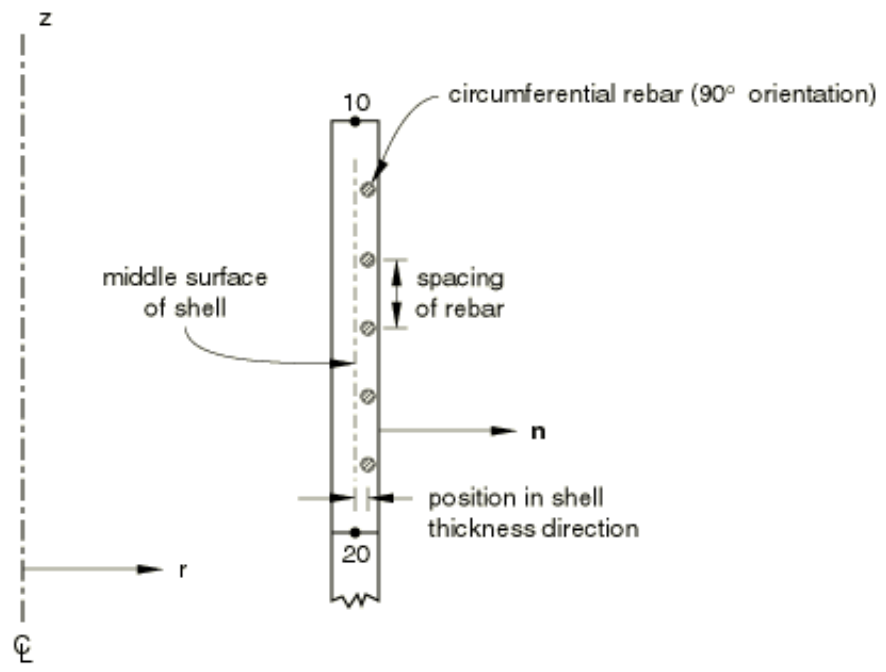

Figure 23. Circumferential rebars in axisymmetric shell elements (Adapted from ABAQUS manual)

ACI 372R-13 suggests a single layer of reinforcement to be used in elements with thickness of $130 \mathrm{~mm}$ or under while two layers are recommended for any thickness greater than this.

The amount of reinforcement should be calculated in accordance with the provisions of the Code. In this case ACI proposes a maximum spacing of $914 \mathrm{~mm}$ and a minimum ratio of non-prestressed reinforcement area-to-concrete area (As/Ac) of 0.0025 in both circumferential and radial directions. Considering these specifications and assuming \#4 bars 
$\left(A_{b a r}=129 \mathrm{~mm}^{2}\right)$, the following reinforcement arrangement is proposed and used for each model:

Table 5. Reinforcement arrangement of the models

\begin{tabular}{|c|c|c|c|c|c|}
\hline Model & $\mathrm{h}(\mathrm{mm})$ & \# Rebar Layers & $\begin{array}{c}\text { Circumferential } \\
\text { Spacing }(\mathrm{mm})\end{array}$ & Angular Spacing $\left(^{\circ}\right)$ & As/Ac \\
\hline 1 & 76 & 1 & 650 & 4.87 & 0.00261 \\
\hline 2 & 76 & 1 & 650 & 2.43 & 0.00261 \\
\hline 3 & 76 & 1 & 650 & 4.88 & 0.00261 \\
\hline 4 & 76 & 1 & 650 & 2.44 & 0.00261 \\
\hline 5 & 102 & 1 & 500 & 1.25 & 0.00253 \\
\hline 6 & 102 & 1 & 500 & 1.07 & 0.00253 \\
\hline 7 & 102 & 1 & 500 & 1.88 & 0.00253 \\
\hline 8 & 89 & 1 & 550 & 1.38 & 0.00264 \\
\hline 9 & 95 & 1 & 500 & 1.07 & 0.00272 \\
\hline 10 & 95 & 1 & 500 & 0.94 & 0.00272 \\
\hline 11 & 114 & 1 & 450 & 0.68 & 0.00251 \\
\hline 12 & 146 & 2 & 700 & 0.88 & 0.00252 \\
\hline 13 & 190 & 2 & 500 & 0.54 & 0.00272 \\
\hline 14 & 235 & 2 & 400 & 0.38 & 0.00274 \\
\hline
\end{tabular}

\subsection{Linear analysis}

For many years the classical buckling equation was the leading formulation for analyzing the instability failure of shell structures; however, after a great deal of debate around this topic, it was known that this equation did not yield accurate results as a consequence of the diverse assumptions made in developing this formulation.

However, to verify the adequacy of the proposed linear finite element technique and also for better understanding of the problem, a linear FE buckling analysis as per the classical buckling formulation will be undertaken. Analysis is performed assuming a linear behavior 
of the material, perfect geometry of the dome and small deformations. The obtained FE results are then compared with the analytical solution proposed by Timoshenko (1936).

\subsubsection{Linear eigenvalue buckling analysis}

The classical buckling formulation estimates the bifurcation buckling of shell structures; however, as expressed by Bushnell (1981), this kind of buckling behavior only occurs in deep perfect spherical shells; while, for the shallow ones, the axisymmetric snapthrough buckling is the expected behavior.

However, to confirm this theory and verify the validity of the proposed numerical technique, all models are subjected to an eigenvalue buckling analysis assuming a linear elastic buckling behavior and a perfect geometric condition in an effort to capture the bifurcation buckling response of the models, covering a wide range of geometries typically found in today's practice.

ABAQUS offers an analysis module called "Buckle" that allows the determination of this particular type of buckling. The process consists of a classical eigenvalue problem whose purpose is to find the loads for which the model stiffness matrix becomes singular:

$$
\left(\left[\mathrm{K}_{0}\right]+\lambda\left[\mathrm{K}_{\Delta}\right]\right) v=0
$$

In this equation, the first matrix $\left[\mathrm{K}_{0}\right]$ represents the stiffness at the initial state, which takes into account the effect of any load previously applied to the model; the second one $\left[\mathrm{K}_{\Delta}\right]$ is the differential stiffness matrix due to the incremental loading, while $\nu$ and $\lambda$ are the corresponding mode shapes and eigenvalues, respectively.

The last mentioned factor $(\lambda)$ multiplied by the perturbation load (Q) plus the load applied at the initial state $(\mathrm{P})$ is set equal to the critical buckling load $\left(q_{c r}\right)$, which in our case 
represents the bifurcation buckling pressure, also known as the classical buckling pressure:

$$
P+(\lambda Q)=q_{c r}
$$

Figure 24 shows Model 1 modelled in accordance with the geometry and material properties mentioned in the preceding sections. For the purpose of analysis, no previous loading is considered; however, as can be observed from the figure, a uniform pressure normal to the surface and equal to unity is applied on the entire structure. As a result, the eigenvalue obtained directly represents the classical buckling pressure of the structure. . This result, as well as the deformed shape of the structure in a bifurcation buckling state can be observed in Figure 25.

A similar procedure is undertaken for all 14 models; however, for the sake of brevity only the finite element idealization of Model 1 is presented. All obtained results from FE analysis are summarized in Table 6.

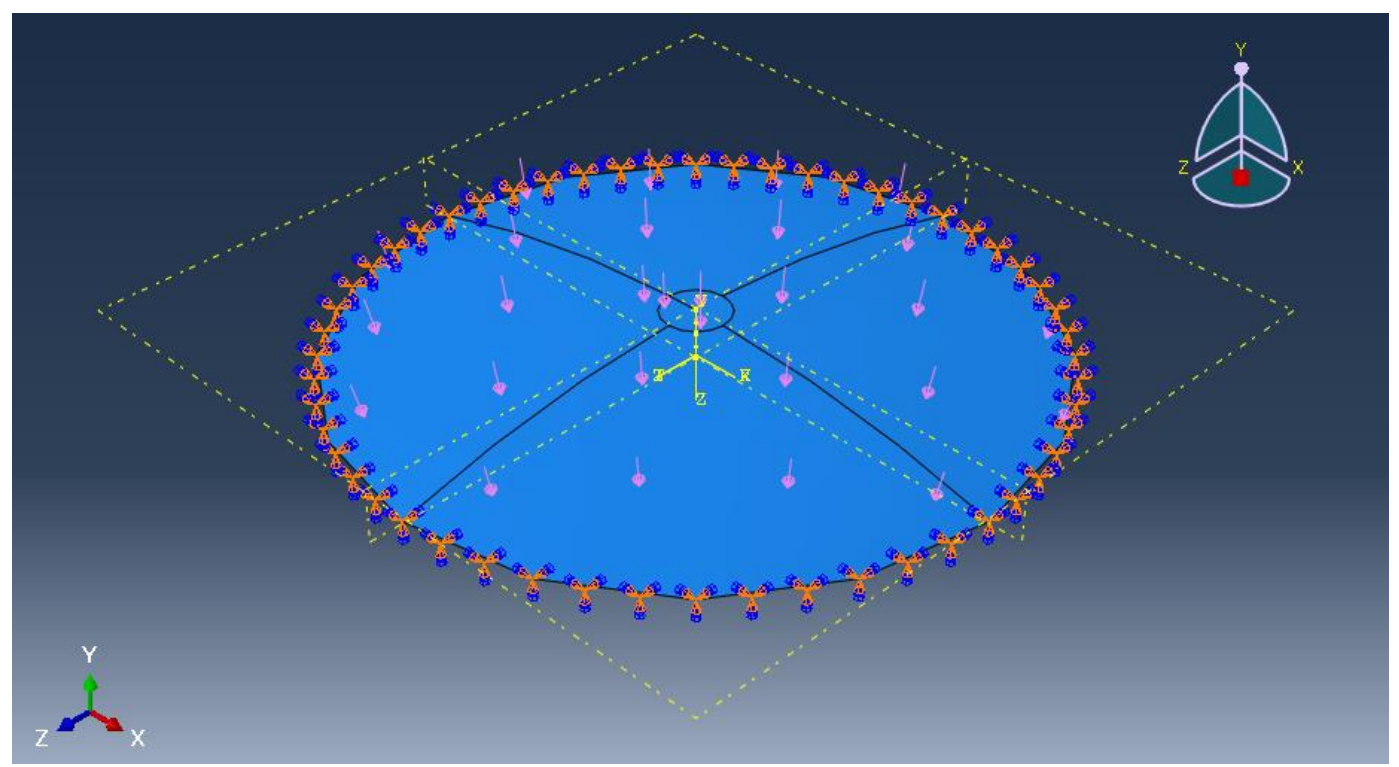

Figure 24. FE idealization of Model 1 


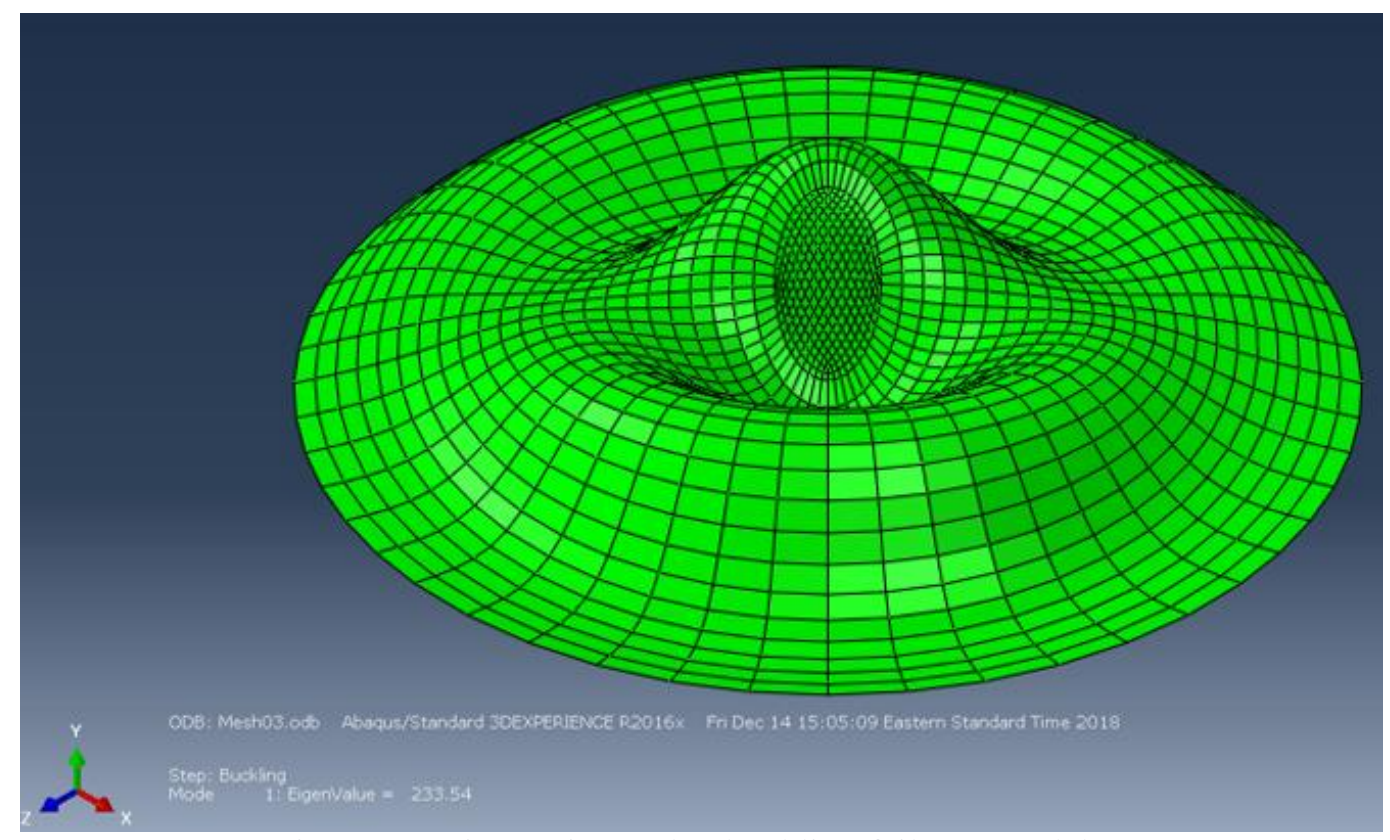

Figure 25. Linear eigenvalue buckling failure (Model 1)

Table 6. Summary of FE buckling analysis results (linear theory)

\begin{tabular}{|c|c|}
\hline \multirow{2}{*}{ Model } & $\begin{array}{c}\text { Classical buckling pressure (FE) } \\
(\mathrm{kPa})\end{array}$ \\
\hline 1 & 233.5 \\
\hline 2 & 59.3 \\
\hline 3 & 268.9 \\
\hline 4 & 68.5 \\
\hline 5 & 54.6 \\
\hline 6 & 39.9 \\
\hline 7 & 201.0 \\
\hline 8 & 67.7 \\
\hline 9 & 56.1 \\
\hline 10 & 64.5 \\
\hline 11 & 59.9 \\
\hline 12 & 70.6 \\
\hline 13 & 86.1 \\
\hline 14 & 103.7 \\
\hline & \\
\hline
\end{tabular}




\subsubsection{Comparison with classical buckling equation}

As initially stated, this linear analysis was performed to be evaluated and contrasted against the results of the classical formulation; therefore in this section a comparison study is carried out between the buckling pressures obtained from both methods and later analyzed in order to estimate the accuracy of the results.

For the determination of the classical buckling, the equation requires the following data to be entered for each model: the radius and thickness of the structure, Poisson's ratio, and elastic modulus of the material. Since we are using a composite material, it is also preferred to use composite material properties; to this effect, the rule of mixture will be used.

This method offers a simple formulation allowing the combination of two materials according to their modulus of elasticity and cross-sectional areas, as indicated in the following equation:

$$
E=\rho \mathrm{E}_{S}+(1-\rho) \mathrm{E}_{C}
$$

Where, $\rho$ is the non-prestressed reinforcement ratio, and $E_{S}$ and $E_{C}$ are the elastic modulus of steel and concrete, respectively. By taking into account the above mentioned material properties, the composite modulus leads to a value of 25,466 MPa. For the case of the Poisson's ratio, the one from concrete material is used since the rule of mixture does not yield a substantial change to it.

Following the classical buckling formulation and on the basis of the above considerations, the results are obtained and summarized in Table 7 and Figure 26. The error from the previously estimated FE results is calculated in accordance with equation 33. 
Table 7. Comparison of linear buckling pressure (FE vs. classical buckling equation)

\begin{tabular}{|c|c|c|c|}
\hline Model & $\begin{array}{c}\text { Classical Buckling Pressure } \\
(\mathrm{kPa})\end{array}$ & $\begin{array}{c}\text { FE } \\
(\mathrm{kPa})\end{array}$ & $\begin{array}{c}\text { Error } \\
(\%)\end{array}$ \\
\hline 1 & 232.6 & 233.5 & $-0.39 \%$ \\
\hline 2 & 58.2 & 59.3 & $-1.89 \%$ \\
\hline 3 & 268.2 & 268.9 & $-0.26 \%$ \\
\hline 4 & 67.1 & 68.5 & $-2.09 \%$ \\
\hline 5 & 53.7 & 54.6 & $-1.68 \%$ \\
\hline 6 & 39.4 & 39.9 & $-1.27 \%$ \\
\hline 7 & 197.8 & 201.0 & $-1.62 \%$ \\
\hline 8 & 66.9 & 67.7 & $-1.20 \%$ \\
\hline 9 & 56.0 & 56.1 & $-0.18 \%$ \\
\hline 10 & 64.2 & 64.5 & $-0.47 \%$ \\
\hline 11 & 59.2 & 59.9 & $-1.18 \%$ \\
\hline 12 & 67.4 & 70.6 & $-4.75 \%$ \\
\hline 13 & 83.8 & 86.1 & $-2.74 \%$ \\
\hline 14 & 98.2 & 103.7 & $-5.60 \%$ \\
\hline
\end{tabular}

$\multimap$ Classical Buckling Pressure $\quad \because$ Abaqus Linear Analysis

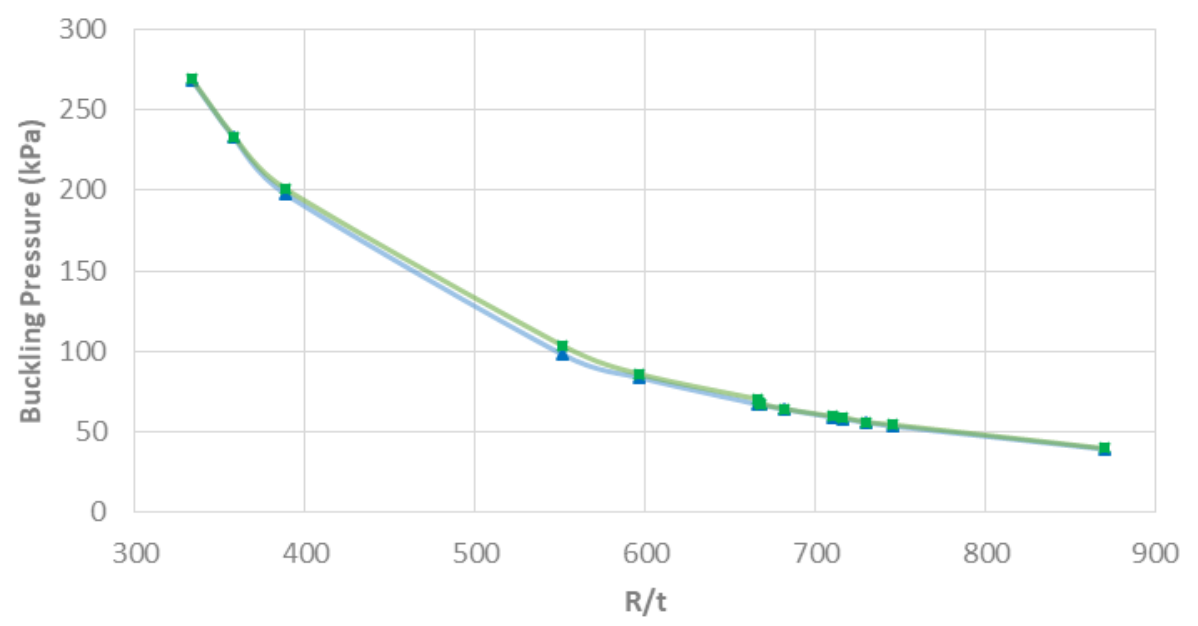

Figure 26. Comparison of linear buckling pressure (FE vs. classical buckling equation)

It is observed that there is a very good correlation between the FE and analytical approaches with the maximum deviation being less than $6 \%$ in all cases. It can also be noted 
that FEM has led to higher buckling pressures in every case.

The difference lies in the fact that: firstly, the finite element method provides accurate but not exact results; therefore a certainty corresponding to zero error is highly unlikely to be achieved, especially in such a complex problem. Secondly, as is also the case in reality, the software is considering a higher amount of steel for the upper sections of the dome. This is due to the fact that typically in these structures because of their geometry, the rebar to concrete ratio is minimum at the base, increases considerably moving toward the higher sections resulting in turn in an increase in the structural capacity. Unfortunately, such effect cannot be taken into account accurately through the classical equation and so such discrepancy is inevitable.

In a similar manner, it is noted that the highest difference between the two sets of data is found for the last three models. The common aspect of these three models is that they all have two layers of reinforcement, in contrast to the rest of the models where only a single layer is provided. Therefore, such deviation in results indicates that the location of reinforcement in the cross section of a shell structure can have an important effect on its capacity, which in this case has represented an increase of up to $6 \%$.

Neglecting the last three models, one can observe that the maximum error obtained is around $2 \%$ which shows a reasonable agreement between the two methods, verifying the accuracy of the proposed numeral model.

\subsection{Nonlinear analysis}

In previous sections, no source of nonlinearity was included in the study. However, as stated earlier, nonlinearity effects such as material nonlinearity, geometric imperfection, and large displacement considerations play an important role in the derivation of actual critical buckling response.

According to Bushnell (1981), the collapse of a spherical cap substantially depends 
on the level of imperfection, and based on the results of numerous investigations the least resistant spherical cap is the one with a shallowness parameter $(\lambda)$ of 4 . Therefore, considering a geometry imperfection level corresponding to this shallowness parameter is expected to result in the most critical buckling pressure for the structure under consideration.

In the same vein, it is also known from these investigations that the bifurcation buckling is not the type of behavior expected to cause failure in structures with this geometric configuration, instead it is proven that for structures with a shallowness parameter of 4 , a snap-through behavior is always experienced. Consequently, a bifurcation buckling analysis is not an appropriate strategy to simulate the response of this class of structures, and so a different approach should be employed. ABAQUS proposes a nonlinear path analysis called "Static Riks" for such problems offering a precise solution for this specific case. More details about this procedure is provided under section 4.5.2.

For the sake of brevity, and since it is out of the scope of this study, the effect of material nonlinearity is not taken into account in the derivation of the buckling pressure. Such effect is not considered in the formulation either. It is important to note that this is a comparative study and all the models are simulated under the same assumptions, as a result of which the obtained results are readily comparable.

\subsubsection{Imperfection}

A nonlinear buckling analysis is performed for the case of imperfect spherical shells. In accordance with the conditions expressed in previous sections, an imperfection level corresponding to the shallowness parameter of 4 is assumed, which is represented by the following span $(\mathrm{d})$ and radius $\left(\mathrm{R}_{\mathrm{imp}}\right)$ for the shell, respectively:

$$
d=4.3 \sqrt{R_{\text {imp }} \times t}
$$




$$
R_{\text {imp }}=1.4 \mathrm{R}
$$

To better understand the effect of this parameter on the buckling response of the domes, Model 1 is analyzed considering two different levels of imperfection and thus two different shallowness parameters; 2 and 4 . Shallowness parameter, $\lambda=2$ corresponds to the following diameter:

$$
d=2.5 \sqrt{R \times t}
$$

As shown in Table 8, the obtained results are in agreement with those from the previously mentioned theory, therefore, the rest of the study will be based on an imperfection level corresponding to a shallowness parameter of 4. For instance, a dome having the geometry properties of Model 1, with a mean radius (R) of $27.22 \mathrm{~m}$ and a shell thickness (h) of $76 \mathrm{~mm}$, would have a diameter $(d)$ in the imperfect region of $7.32 \mathrm{~m}$ and a radius $\left(R_{\text {imp }}\right)$ of $38.11 \mathrm{~m}$, in accordance with the configuration described in Figure 27.

Table 8. Effect of different levels of geometric imperfections

\begin{tabular}{|c|c|c|c|}
\hline \multirow{3}{*}{ Model } & $\begin{array}{c}\text { Analytical nonlinear } \\
\text { Buckling Pressure } \\
(\mathrm{kPa})\end{array}$ & $\begin{array}{c}\text { FE Buckling Pressure } \\
\lambda=4 \\
(\mathrm{kPa})\end{array}$ & $\begin{array}{c}\text { FE Buckling Pressure } \\
\lambda=2\end{array}$ \\
& 65.04 & 65.4 & $(\mathrm{kPa})$ \\
\hline 1 & & & 120.8 \\
\hline
\end{tabular}

In a similar manner, the geometric imperfection parameters for each model are calculated and summarized in Table 9. 


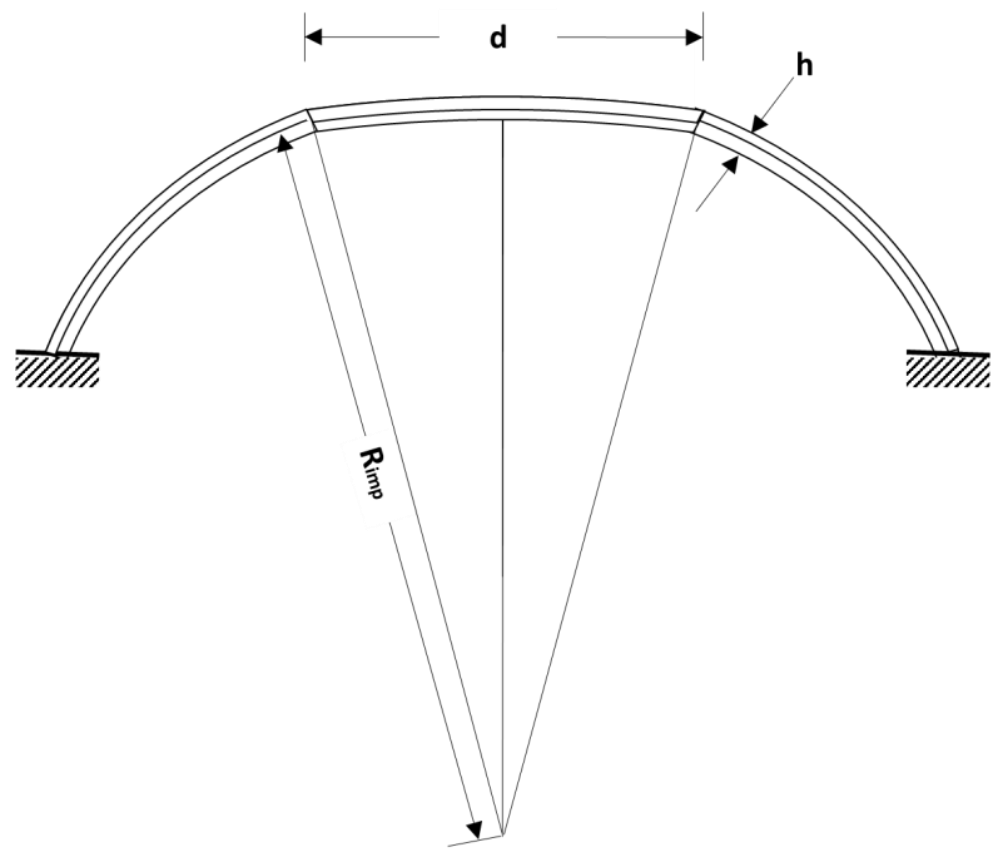

Figure 27. Geometry of the imperfect region

Table 9. Geometric imperfection parameters

\begin{tabular}{|c|c|c|c|c|}
\hline Model & $\begin{array}{c}d \text { (imperfect) } \\
(\mathrm{m})\end{array}$ & $\begin{array}{c}R_{\text {imp }} \text { (imperfect) } \\
(\mathrm{m})\end{array}$ & $\begin{array}{c}\text { Rise(imperfect) } \\
(\mathrm{m})\end{array}$ & $\lambda$ \\
\hline 1 & 7.32 & 38.11 & 0.1753 & 4.0 \\
\hline 2 & 10.35 & 76.20 & 0.1755 & 4.0 \\
\hline 3 & 7.06 & 35.49 & 0.1752 & 4.0 \\
\hline 4 & 9.99 & 70.98 & 0.1754 & 4.0 \\
\hline 5 & 14.17 & 106.47 & 0.2355 & 4.0 \\
\hline 6 & 15.31 & 124.22 & 0.2355 & 4.0 \\
\hline 7 & 10.23 & 55.47 & 0.2352 & 4.0 \\
\hline 8 & 11.70 & 83.22 & 0.2054 & 4.0 \\
\hline 9 & 13.06 & 97.08 & 0.2193 & 4.0 \\
\hline 10 & 12.62 & 90.68 & 0.2193 & 4.0 \\
\hline 11 & 15.46 & 113.34 & 0.2632 & 4.0 \\
\hline 12 & 19.16 & 136.02 & 0.3370 & 4.0 \\
\hline 13 & 23.61 & 158.69 & 0.4385 & 4.0 \\
\hline 14 & 28.07 & 181.36 & 0.5423 & 4.0 \\
\hline
\end{tabular}

These imperfection parameters are now considered in both numerical and analytical 
analyses. For the case of FEA, the imperfections are directly applied to the geometry of the models through the "section sketch" module in ABAQUS during the idealization process. As the analytical approach, the formulation suggested by Zhargamee and Heger (1983) is used. As previously explained in Chapter 2 , they proposed inclusion of a new parameter, $\beta_{\text {imp }}$ in the original buckling equation to account for imperfection effects leading to a considerable reduction in the predicted buckling capacity for imperfect shells, as follows:

$$
\beta_{i m p}=\left[\frac{R / t}{(R / t)_{i m p}}\right]^{2}
$$

Assuming the thickness to be the same under both conditions and thus eliminating that from numerator and denominator, and referring to equation $38, \beta_{\text {imp }}$ is estimated as 0.50 (50\% reduction). Referring to equation 25 and considering $\beta_{i m p}=0.50$ will lead to the derivation of the following equation for the buckling capacity of an imperfect dome assuming a shallowness parameter of $\lambda=4$ :

$$
p_{c r}=0.33 \mathrm{E}\left(\frac{t}{R}\right)^{2}
$$

The resulting buckling pressures according to this formulation are listed in Table 10. The results from the classical linear buckling equation is also given in the table for comparison purposes.

As can be observed from the table, there is a significant reduction in the buckling capacity of the models where the effect of nonlinearity due to imperfection is taken into consideration. As the obtained results suggest such effect can cause up to $72 \%$ reduction in the buckling capacity of the model representing a significant disagreement between the two formulations. This reduction is expected to be even more dramatic where the effect of material nonlinearity is also included. 
Table 10. Analytical linear and nonlinear buckling pressure

\begin{tabular}{|c|c|c|}
\hline Model & $\begin{array}{c}\text { Linear Classical Buckling Pressure } \\
(\mathrm{kPa})\end{array}$ & $\begin{array}{c}\text { Nonlinear Buckling Pressure } \\
(\mathrm{kPa})\end{array}$ \\
\hline 1 & 232.62 & 65.04 \\
\hline 2 & 58.18 & 16.27 \\
\hline 3 & 268.21 & 74.99 \\
\hline 4 & 67.05 & 18.75 \\
\hline 5 & 53.68 & 15.01 \\
\hline 6 & 39.43 & 11.03 \\
\hline 7 & 197.77 & 55.30 \\
\hline 8 & 66.90 & 18.70 \\
\hline 9 & 56.01 & 15.66 \\
\hline 10 & 64.19 & 17.95 \\
\hline 11 & 59.17 & 16.54 \\
\hline 12 & 67.38 & 18.84 \\
\hline 13 & 83.84 & 23.44 \\
\hline 14 & 98.20 & 27.46 \\
\hline
\end{tabular}

\subsubsection{Static Riks Analysis}

The Riks method is a practical tool that allows the prediction of the nonlinear buckling failure of a structure, whether the response is stable or unstable. The procedure is based on a load-deflection analysis that treats both parameters as unknown values; which necessitates the introduction of another parameter, called "arc length" in order to measure the progress of the solution.

The analysis consists of a number of arc length increments, where the first one is set by the user; and the subsequent ones are computed automatically by the software using the Newton's method, following however the minimum and maximum values also set by the user. This will give the user a better control on the automatic incrementation process.

The resulting loading $\left(P_{\text {total }}\right)$ at each increment is described as a proportional load defined by the initial loading $\left(P_{0}\right)$, a load proportionality factor $(\lambda)$ and the reference load 
$\left(P_{\text {ref }}\right)$ as shown in equation 42.

$$
P_{\text {total }}=P_{0}+\lambda P_{\text {ref }}
$$

Where, the initial load has a constant magnitude and comprises any load applied during previous steps; the magnitude of the reference load is defined by the loading of the current step; while the load proportionality factor, LPF, is found as part of the solution for each increment.

For the purpose of this part of the study, no initial loading condition is considered $\left(P_{0}=0\right)$; however, a target load larger than the expected buckling capacity of the model is applied. The peak load proportionality factor $(\lambda)$ occurring right before the first sudden drop in the LPF-Arc Length curve is measured and multiplied by the magnitude of the corresponding reference load $\left(P_{r e f}\right)$ and the calculated value is defined as the nonlinear buckling capacity of the structure.

In Figure $21(\mathrm{a}-\mathrm{n})$ the results obtained from the finite element Riks Analysis are shown; it is noted that all the models show a snap-through unstable post-buckling behavior as anticipated; confirming the consistency with the assumptions initially made. 


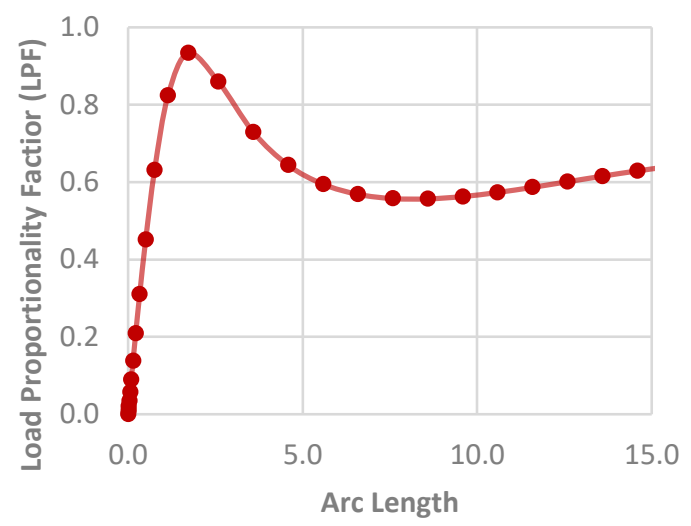

(a) Model 1

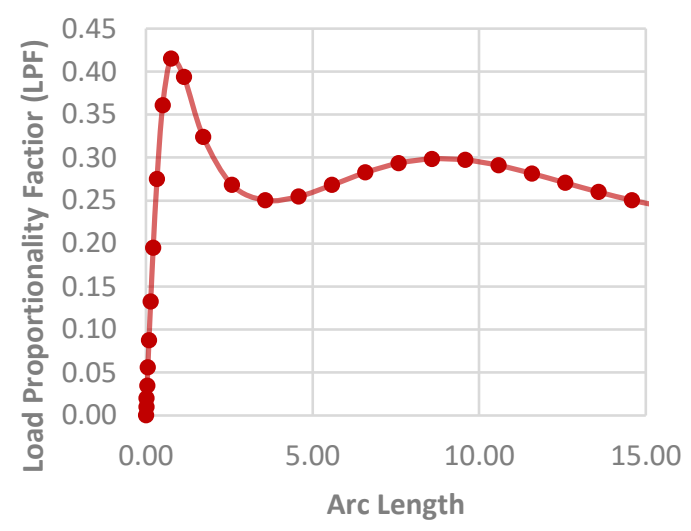

(c) Model 3

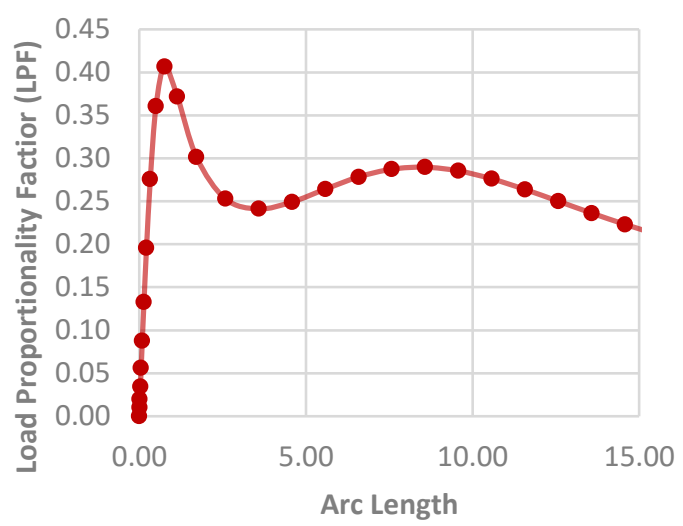

(b) Model 2

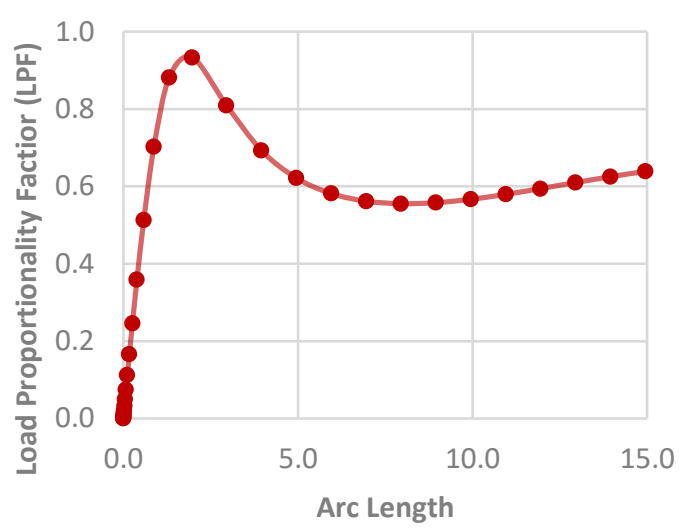

(d) Model 4

Figure 28. Results obtained from FE Static Riks analysis 


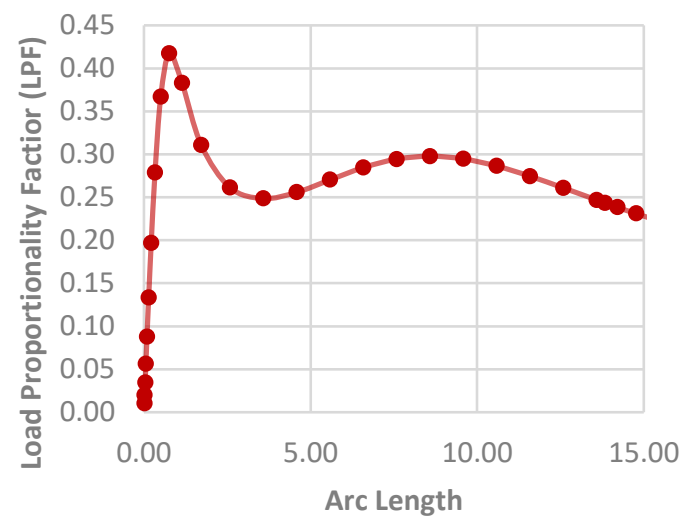

(e) Model 5

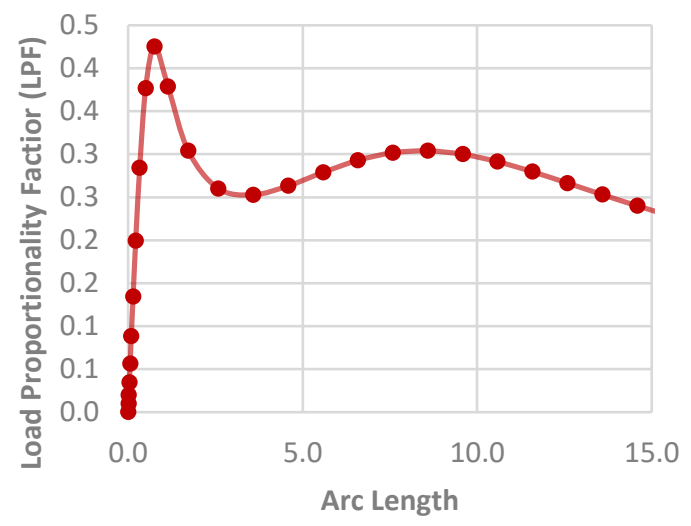

(g) Model 7

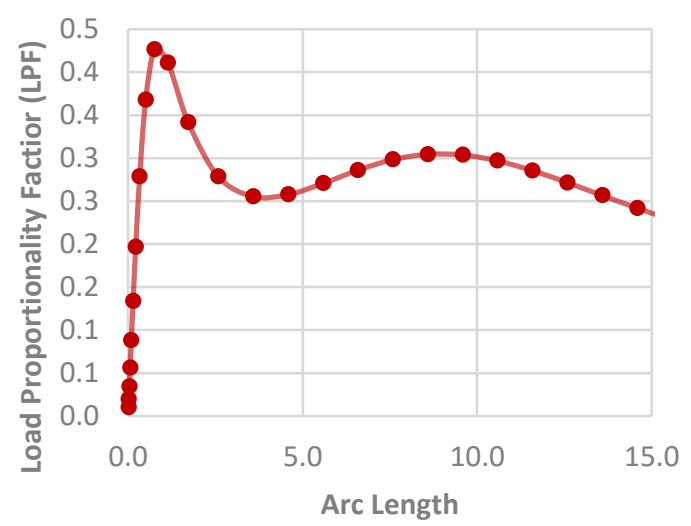

(i) Model 9

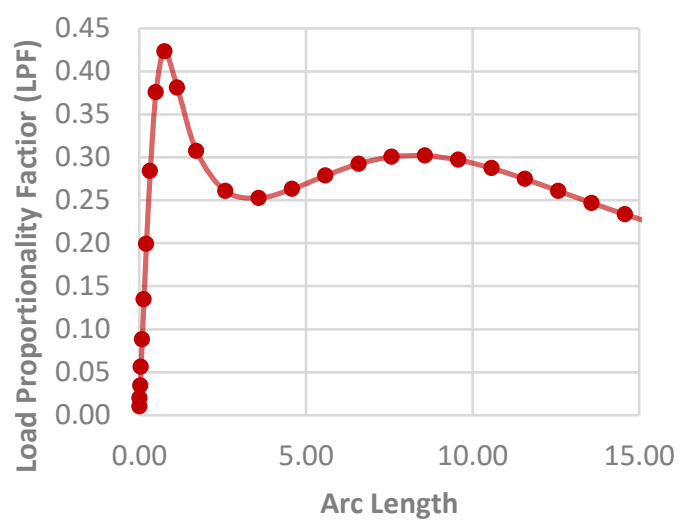

(f) Model 6

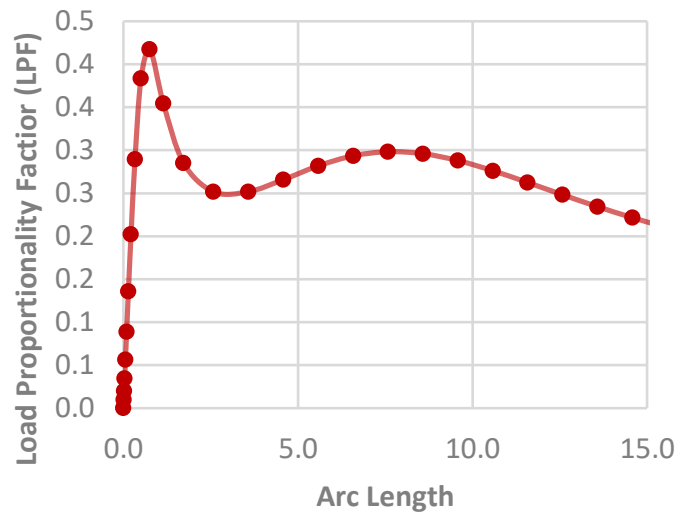

(h) Model 8

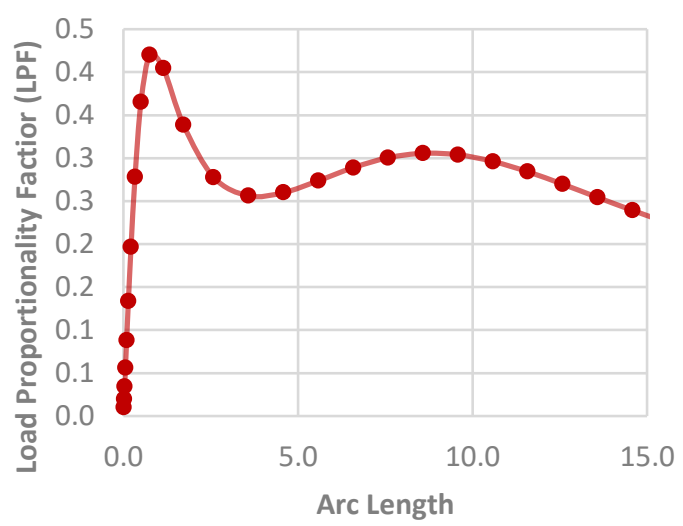

(j) Model 10

Figure 28. Results obtained from FE Static Riks analysis, Continued 


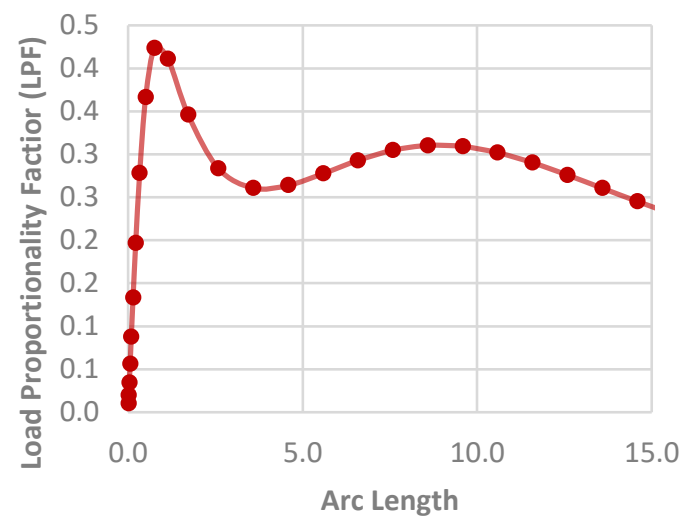

(k) Model 11

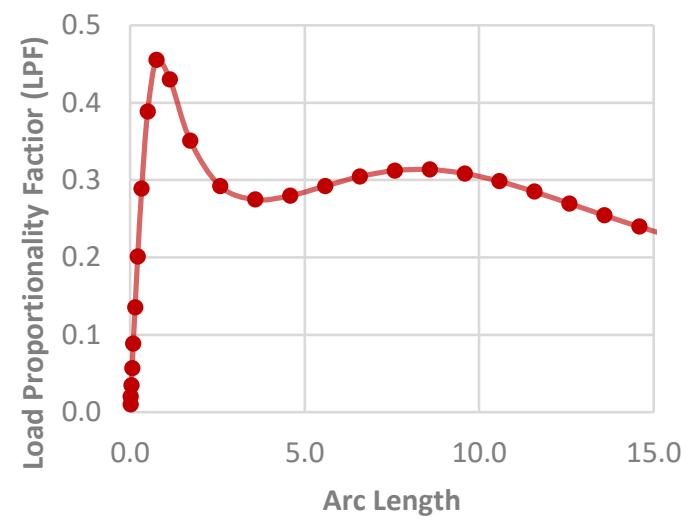

(m) Model 13

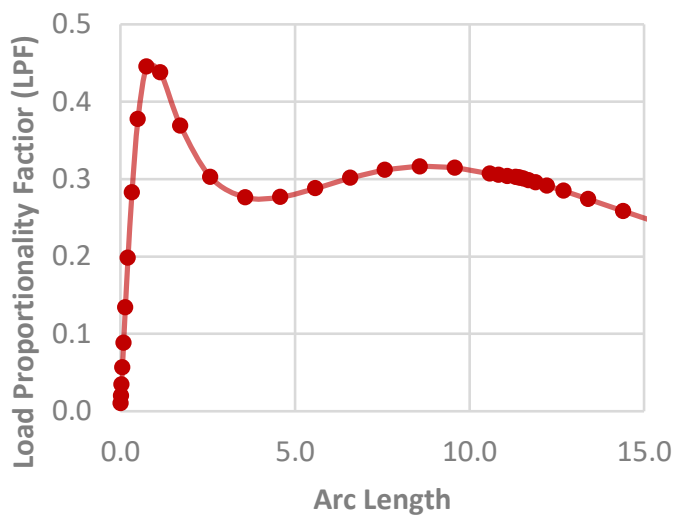

(1) Model 12

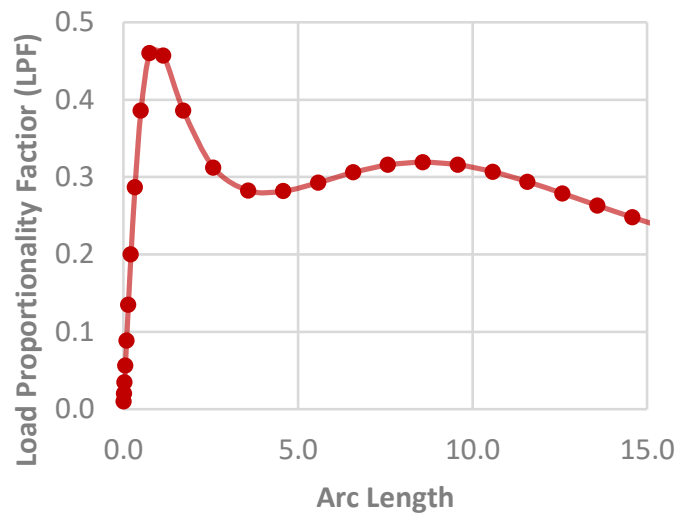

(n) Model 14

Figure 28. Results obtained from FE Static Riks analysis, Continued

In the case of Model 1, at the time of first drop the magnitudes of load proportionality factor and reference pressure are 0.9336 and $70 \mathrm{kPa}$, respectively resulting in a structural buckling capacity of $65.35 \mathrm{kPa}$. Figure 29 shows the corresponding snap-through failure of the structure.

Using the same approach, the buckling capacity for each of the 13 remaining models is calculated and listed in Table 11. 


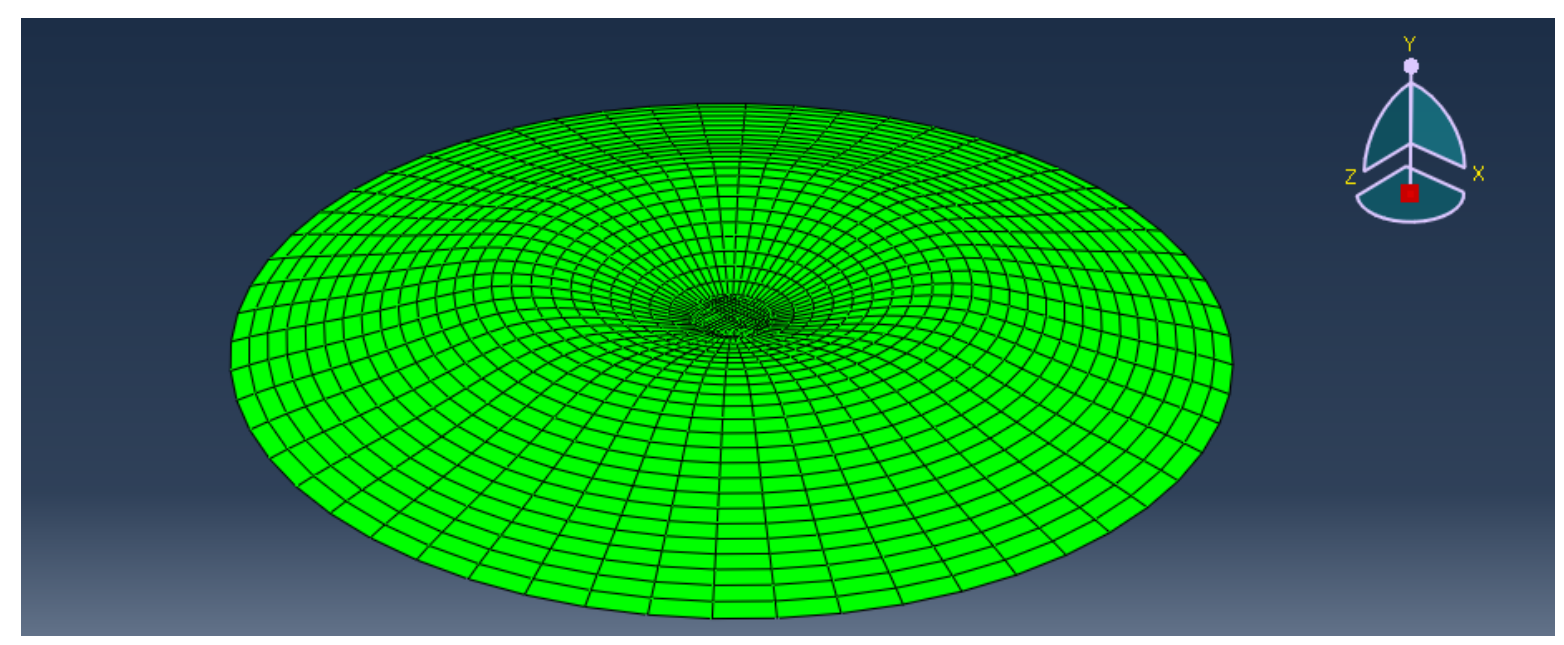

Figure 29. Snap-through buckling failure of Model 1

Table 11. Nonlinear buckling capacity (FE)

\begin{tabular}{|c|c|}
\hline \multirow{2}{*}{ Model } & $\begin{array}{c}\text { Non-linear Buckling Pressure } \\
(\mathrm{kPa})\end{array}$ \\
\hline 1 & 65.35 \\
\hline 2 & 16.26 \\
\hline 3 & 74.69 \\
\hline 4 & 18.67 \\
\hline 5 & 15.02 \\
\hline 6 & 11.00 \\
\hline 7 & 55.30 \\
\hline 8 & 18.78 \\
\hline 9 & 15.77 \\
\hline 10 & 18.07 \\
\hline 11 & 16.53 \\
\hline 13 & 20.04 \\
\hline 14 & 25.94 \\
\hline
\end{tabular}

\subsubsection{Comparison of results with the ACI formulation}

Once again, in order to validate the FE technique, the obtained results are compared with the closed-form solution proposed by Zhargamee and Heger (1983), later adopted by 
ACI.

Table 12 lists the results from ACI under the first column and the FE results under the second column, while the third column displays the percentage of error between the two approaches. In a similar manner, the results are organized with respect to the radius-tothickness ratio and presented in Figure 30.

It is noted that both analytical and numerical strategies have resulted in close estimations of critical buckling pressure, achieving an error of less than 1\% for Models 1 to 11. However, for the last three models the error percentage varies between $6 \%$ and $11 \%$. This discrepancy is attributed again to the arrangement of steel reinforcement in the shell's cross section. As mentioned earlier, these models have two layers of reinforcement instead of one, providing greater resistance against buckling and thus a higher buckling capacity.

Table 12. Nonlinear buckling response (ACI vs. FE)

\begin{tabular}{|c|c|c|c|}
\hline Model & $\begin{array}{c}\text { ACI Buckling } \\
\text { Pressure } \\
(\mathrm{kPa})\end{array}$ & $\begin{array}{c}\text { FE Nonlinear } \\
\text { Buckling Pressure } \\
(\mathrm{kPa})\end{array}$ & $\begin{array}{c}\text { Error } \\
(\%)\end{array}$ \\
\hline 1 & 65.04 & 65.35 & $-0.47 \%$ \\
\hline 2 & 16.27 & 16.26 & $0.06 \%$ \\
\hline 3 & 74.99 & 74.69 & $0.40 \%$ \\
\hline 4 & 18.75 & 18.67 & $0.44 \%$ \\
\hline 5 & 15.01 & 15.02 & $-0.07 \%$ \\
\hline 6 & 11.03 & 11.00 & $0.24 \%$ \\
\hline 7 & 55.30 & 55.30 & $0.00 \%$ \\
\hline 8 & 18.70 & 18.78 & $-0.41 \%$ \\
\hline 9 & 15.66 & 15.77 & $-0.69 \%$ \\
\hline 10 & 17.95 & 18.07 & $-0.67 \%$ \\
\hline 11 & 16.54 & 16.53 & $0.07 \%$ \\
\hline 12 & 18.84 & 20.04 & $-6.38 \%$ \\
\hline 13 & 23.44 & 25.94 & $-10.65 \%$ \\
\hline 14 & 27.46 & 30.37 & $-10.60 \%$ \\
\hline
\end{tabular}




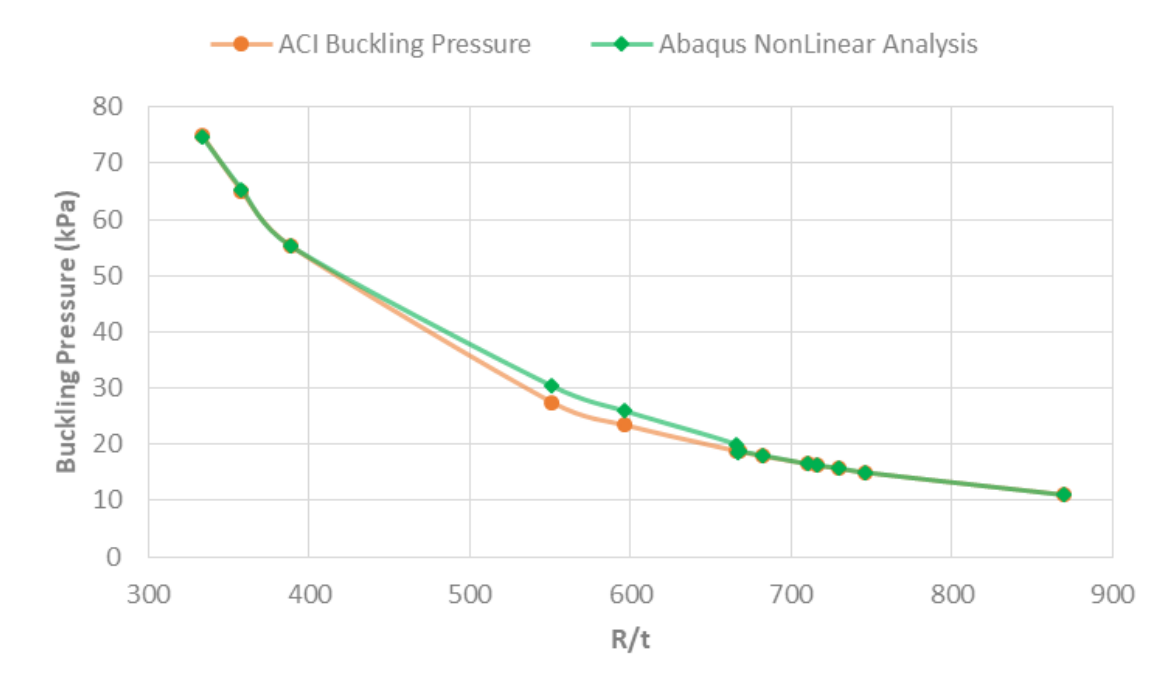

Figure 30. ACI vs. FE nonlinear buckling results

Aside from the above mentioned, no other discrepancy was found in the obtained results; suggesting that the proposed nonlinear FE model can be considered as an accurate representation of the problem.

\subsubsection{Comparison between the bifurcation and snap-through buckling response}

In this part of the study, the numerical results obtained through the linear (bifurcation) and nonlinear (snap-through) buckling approaches are compared and displayed in Table 13 and Figure 31.

It is interesting to note that including the nonlinearity in the analysis of such systems has caused a substantial reduction of between $70 \%$ and $73 \%$ (average of around $72 \%$ ) in the buckling pressure for the models considered in this research. 
Table 13. Bifurcation vs. Snap-through buckling (FE)

\begin{tabular}{|c|c|c|c|}
\hline Model & $\begin{array}{c}\text { Bifurcation Buckling } \\
(\mathrm{kPa})\end{array}$ & $\begin{array}{c}\text { Snap-through Buckling } \\
(\mathrm{kPa})\end{array}$ & $\begin{array}{c}\text { Error } \\
(\%)\end{array}$ \\
\hline 1 & 233.54 & 65.35 & $72 \%$ \\
\hline 2 & 59.291 & 16.26 & $73 \%$ \\
\hline 3 & 268.9 & 74.69 & $72 \%$ \\
\hline 4 & 68.485 & 18.67 & $73 \%$ \\
\hline 5 & 54.637 & 15.02 & $73 \%$ \\
\hline 6 & 39.949 & 11.00 & $72 \%$ \\
\hline 7 & 201.03 & 55.30 & $72 \%$ \\
\hline 8 & 67.684 & 18.78 & $72 \%$ \\
\hline 9 & 56.111 & 15.77 & $72 \%$ \\
\hline 10 & 64.49 & 18.07 & $72 \%$ \\
\hline 11 & 59.905 & 16.53 & $72 \%$ \\
\hline 12 & 70.584 & 20.04 & $72 \%$ \\
\hline 13 & 86.074 & 25.94 & $70 \%$ \\
\hline 14 & 103.74 & 30.37 & $71 \%$ \\
\hline
\end{tabular}

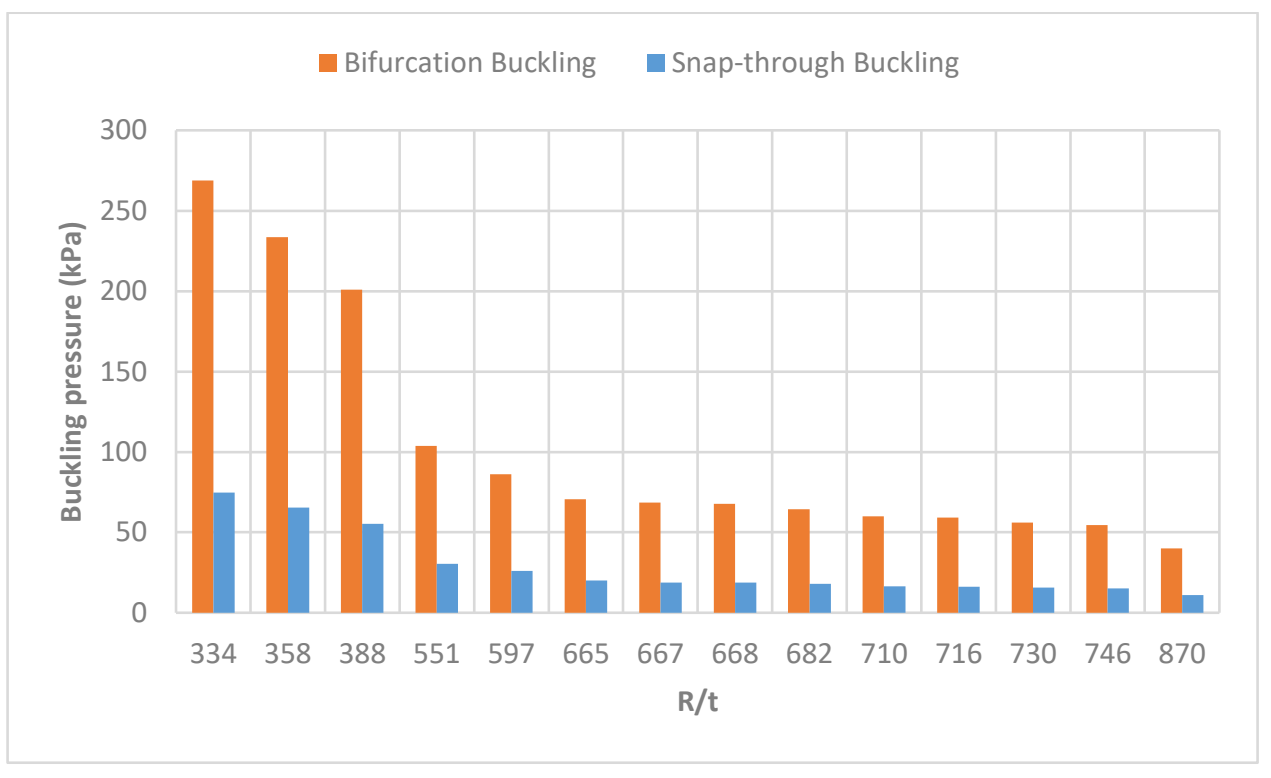

Figure 31. Bifurcation vs. Snap-through buckling (FE) 
It is observed that despite the use of different Radius-to-thickness ratios, the difference between linear and nonlinear analysis is constant. This however is the logical and expected result, since in all the cases an imperfection corresponding to the size of a shallowness parameter of four was used. And since according to Bushnell (1967) the failure of the structure occurs in the area of the shell with the imperfection; when using the same size, then, similar behavior is expected in all cases.

\subsubsection{Effect of base fixity}

The effect of base boundary condition on the buckling pressure of spherical shells has been extensively investigated in the past and the obtained results suggest that the most critical case is caused as a result of a shallowness parameter $(\lambda)$ of 4 and a clamped boundary condition. The studies further suggest that for values of $\lambda$ higher than 5 , a simply supported boundary condition may lead to lower buckling capacities. However, considering that in this case two different $\lambda$ are involved in the model; the one corresponding the perfect shell and the other from the imperfect region; the impact of the boundary conditions on the buckling failure of the structure cannot be predicted; since the combined effect of two shallowness parameter and different edge conditions have not yet been addressed in previous investigations.

On the other hand, the clamped boundary condition is considered to be the most accurate representation of the actual connection between the wall and the roof. However, to provide better understanding of the subject, the effect of having different base fixities on the buckling capacity of shell structures is investigated in this part. To do so, models with the same exact properties and geometries but hinged base boundary connection are recreated and analyzed, and the measured results are compared against those of fixed boundary condition in Table 14 and in Appendix A, Figures A. 1-14. 
Table 14. Effect of base fixity on buckling capacity (FE)

\begin{tabular}{|c|c|c|c|}
\hline \multirow{2}{*}{ Model } & Fixed Base & Hinged Base & Error \\
& $(\mathrm{kPa})$ & $(\mathrm{kPa})$ & $(\%)$ \\
\hline 1 & 65.35 & 64.13 & $1.87 \%$ \\
\hline 2 & 16.26 & 16.28 & $-0.14 \%$ \\
\hline 3 & 74.69 & 73.37 & $1.77 \%$ \\
\hline 4 & 18.67 & 18.72 & $-0.27 \%$ \\
\hline 5 & 15.02 & 15.02 & $-0.01 \%$ \\
\hline 6 & 11.00 & 11.00 & $0.03 \%$ \\
\hline 7 & 55.30 & 55.38 & $-0.15 \%$ \\
\hline 8 & 18.78 & 18.79 & $-0.09 \%$ \\
\hline 9 & 15.77 & 15.75 & $0.11 \%$ \\
\hline 10 & 18.07 & 18.06 & $0.08 \%$ \\
\hline 11 & 16.53 & 16.51 & $0.09 \%$ \\
\hline 12 & 20.04 & 20.02 & $0.11 \%$ \\
\hline 13 & 25.94 & 25.91 & $0.08 \%$ \\
\hline 14 & 30.37 & 30.33 & $0.13 \%$ \\
\hline
\end{tabular}

From the table and the graphs, one can notice that there is no consistent pattern in the obtained results inferring which type of boundary condition is more critical than the other, and indeed, the results are surprisingly close suggesting that the base fixity condition (fixed or hinged) has no significant effect on the buckling behavior.

Additionally, it is noticed that changing the type of boundary condition from fixed to hinged does not change the structure's mode of buckling failure, as all models keep their unstable snap-through post-buckling behavior.

On the other hand, the formulation proposed by ACI does not distinguish between hinged or fixed boundary conditions and predicts the same buckling capacity for both base conditions. Upon examining Figure 32, it can be seen that in most cases a fixed boundary condition produces closer results to the ones estimated by ACI. 


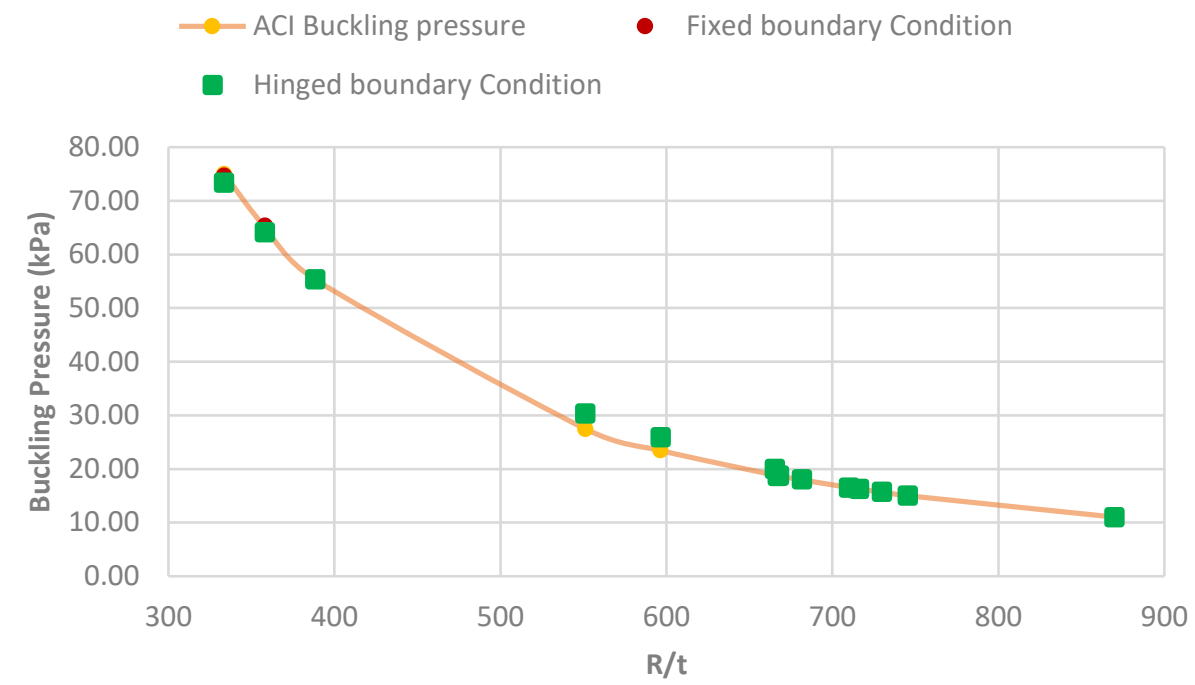

Figure 32. Comparison between ACI and FE for different types of boundary conditions

Based on the obtained results, it can be concluded that the buckling pressure in spherical shell structures is not highly influenced by the boundary condition effects when an imperfection corresponding to $\lambda=4$ is in place. In the present work a clamped boundary condition will be used in the subsequent chapters.

\subsection{Summary}

In this chapter, the buckling capacity of different dome configurations was determined under both linear and nonlinear conditions using the finite element technique proposed in the previous chapter. The results were later compared with the corresponding analytical solution in order to validate the finite element method. At the same time the effects of different parameters such as: geometric configuration, reinforcement arrangement, imperfection, and base fixity were also addressed.

At the beginning, 14 different geometric configurations were presented. This was achieved by varying the thickness, radius, and angle of the domes providing then a wide range of geometries found in practice today. Once the analysis was carried out, it was noted 
that as expected the lower the radius-to-thickness ratio, the higher the buckling capacity; setting a clear behavior with respect to this parameter.

With regards to the base fixity effect, a nonlinear analysis was performed on all 14 models using both fixed and hinged restraints at the edge of the dome. No considerable difference in buckling response was experienced as a result of eliminating the rotational restriction at the base. Overall, there was not a specific pattern associated with the two different boundary conditions considered, as in some cases an increase and in others a decrease in buckling pressure was observed. However, despite the lack of uniformity in the results, a fixed boundary condition is considered adequate for the idealization of the problem and will therefore be used in future chapters.

It was also concluded that placing the steel reinforcement in two layers instead of one, despite representing the same area of reinforcement, increases the buckling capacity of the structure. Unfortunately, such effect cannot be accurately predicted by the existing analytical approach adopted in ACI; however, the Code results were always on the conservative side and thus suitable for design purposes.

It was indicated by Bushnell (1981) and was reaffirmed by Zhargamee (1983) that the most critical case corresponded to a spherical shell with a shallowness parameter of 4 . The validity of this theory was further supported by the results obtained from FE analysis in this study. As a result, a shallowness parameter of 4 will be considered in buckling response analysis of the models in the following chapters as well.

For the case of linear buckling, an eigenvalue buckling analysis was performed using ABAQUS, considering a linear material, a perfect geometry, small displacements and constant thickness, as proposed by Timoshenko (1936). It was observed that numerical analysis led to higher results in all cases. This was influenced by the ratio of reinforcement considered in the analytical formulation in comparison to the numerical idealization. However, despite this slight difference, there was a high level of correlation between the 
results. The classical buckling equation was also found to always lead to a conservative response when dealing with linear conditions.

On the other hand, the nonlinearity effects due to geometry imperfections and large deformations/displacements were also included in analysis of the shells; however, with respect to material properties, only a linear elastic behavior was considered. The obtained results showed a clear agreement between the numerical and analytical models. As a result, the validity of the proposed finite element procedure was verified under both linear and nonlinear conditions.

Comparing the linear and nonlinear analytical formulations, a nearly constant average reduction of $72 \%$ was observed in the buckling capacity of the models due to nonlinearity effects; indicating once again the large disagreement between the two strategies. However, comparing these results with the obtained FE numbers show that each formulation is accurate under its own terms. But then again, it should be made clear that the classical buckling equation does not represent the reality of the behavior of such structures, unlike the formulation by Zarghamee (1983) better describing the actual buckling behavior of the shell and also agreeing well with both experimental and FE results. 


\section{CHAPTER 5}

\section{BUCKING RESPONSE OF SPHERICAL SHELLS SUBJECTED TO EARTHQUAKE LOADING}

\section{$5.1 \quad$ Introduction}

In this chapter, the buckling response of spherical shells under seismic loading is analyzed using the finite element technique verified in previous chapters. However, this time new procedures are also incorporated into the analysis for the determination of seismic effects.

The main objective of this chapter is not only to study the buckling of spherical shell structures subjected to general earthquake loading, but first and foremost, to specifically focus on the effect of its horizontal component. To this effect, the analysis is carried out in two parts; first, where only the vertical earthquake component is applied in combination with the self-weight of the shell; and second, considering the same loading conditions but this time adding the horizontal component as well. Once the results from both parts are obtained, they can be analyzed and compared with each other, taking into consideration the influence that other parameters could also have on the response.

However, prior to this, the respective seismic loading to be applied to the structure should be first determined, for which the response spectrum analysis proposed by ASCE710 is considered suitable. For the purpose of spectral analysis, the first challenge is to obtain the fundamental frequency of the structure. To this end, there has been a number of analytical solutions for this particular type of structure; however, in these formulations the effect of imperfection is not included. As previously discussed such effect can represent an important contribution to the response and neglecting that may result in large discrepancies. Therefore, once again, finite element method as a useful tool capable of providing a precise solution to this problem is employed. The method allows to perform a free vibration analysis and obtain 
the natural frequency of complex configurations, needed for the subsequent determination of the horizontal seismic loading.

The vertical component is determined in accordance with both ASCE7-10 and ACI372R-13. Since the definition of vertical component is different in each case, two different Eh/Ev ratios are found, implying different levels of participation of horizontal component. This represents a new factor of interest to be studied through this research.

Finally, the second focus of this chapter is to determine the accuracy of ACI approach when considering the seismic effects. To this end, the buckling capacity under seismic action estimated by the proposed finite element method is compared with that from the analytical formulation proposed by ACI.

\subsection{Natural frequency extraction}

As it is known, the fundamental frequency of a structure corresponds to the lowest natural frequency that the structure could experience. In this study, the extraction of these

frequencies is necessary for each model, in order to determine the corresponding maximum spectral response acceleration of the structure.

In the literature, there are diverse formulations proposed to determine this frequency; however, neither of them takes into account the imperfection in the structure, which, as explained before can highly influence the results obtained. In this study, the extraction of the fundamental frequencies of the imperfect domes has been made possible using the FEA software, ABAQUS and through its powerful analysis module called FREQUENCY.

The module offers a linear perturbation procedure that is capable of solving the eigenvalue problem for symmetric mass and stiffness matrices; obtaining for each case the frequency and its corresponding mode shape. A classical eigenvalue undamped finite element model is represented by the following equation: 


$$
\left(-\omega^{2}[\mathrm{M}]+[\mathrm{K}]\right) \lambda=0
$$

Where, $[\mathrm{M}]$ represents the mass matrix, $[\mathrm{K}]$ the stiffness matrix, $\omega$ the frequencies and $\lambda$ the eigenvector or mode of vibration. ABAQUS proposes different types of methods to solve this formulation: Lanczos, Automatic Multi-level Substructuring (AMS), and Subspace iteration. For this investigation, the Lanczos eigensolver is selected, since it is a faster solver for systems with many degrees of freedom. The obtained free vibration FE results are listed in Table 15 for the 14 models discussed in previous chapters:

Table 15. Free vibration analysis results

\begin{tabular}{|c|c|c|}
\hline Model & $\begin{array}{l}\text { Fundamental Frequency } \\
\qquad(1 / \mathrm{s})\end{array}$ & $\begin{array}{c}\text { Fundamental Period } \\
\text { (s) }\end{array}$ \\
\hline 1 & 15.091 & 0.066 \\
\hline 2 & 7.459 & 0.134 \\
\hline 3 & 16.161 & 0.062 \\
\hline 4 & 7.993 & 0.125 \\
\hline 5 & 5.324 & 0.188 \\
\hline 6 & 4.577 & 0.218 \\
\hline 7 & 10.221 & 0.098 \\
\hline 8 & 6.777 & 0.148 \\
\hline 9 & 5.753 & 0.174 \\
\hline 10 & 6.071 & 0.165 \\
\hline 11 & 4.864 & 0.206 \\
\hline 12 & 4.117 & 0.243 \\
\hline 13 & 3.561 & 0.281 \\
\hline 14 & 3.122 & 0.320 \\
\hline
\end{tabular}

As expected the domes with larger mass and higher rise produce higher periods and 
consequently lower frequencies.

\subsection{Horizontal component of earthquake}

To determine the magnitude of the horizontal component of earthquake, the Code ASCE 7-10 is used.

Initially the site class and the location are selected in order to obtain the corresponding seismic parameters. Site class D is assumed as suggested by ASCE for the case where there is no geotechnical study available; while Missouri is the location assumed for the study as it is classified as one of the highest seismic zones in the USA according to the national seismic hazard mapping project website.

According to these assumptions and using the seismic hazard mapping project website, the values of $S s$ and $S_{1}$ are determined as 2.74 and 1.084, respectively. Since the current investigation is not focused on the design of the structure, the values of $\mathrm{I}=1$ (Importance Factor) and $\mathrm{R}=1$ (Response modification factor) are chosen for the spectral analysis.

The design spectral response acceleration $S_{a}$ is determined as follows as per ASCE 7-10:

$$
\begin{array}{lc}
\text { For } T<T_{0} & S_{a}=S_{D S}\left(0.4+0.6 \frac{T}{T_{0}}\right) \\
\text { For } T_{0}<T \leq T_{S} & S_{a}=S_{D S} \\
\text { For } T_{S}<T \leq T_{L} & S_{a}=\frac{S_{D 1}}{T}
\end{array}
$$


For $T>T_{L}$

$$
S_{a}=\frac{S_{D 1} T_{L}}{T^{2}}
$$

Where,

$\mathrm{S}_{\mathrm{DS}}=\frac{2}{3} \mathrm{~F}_{a} \mathrm{~S}_{S}=$ Design spectral response acceleration parameter at short periods

$\mathrm{S}_{\mathrm{D} 1}=\frac{2}{3} \mathrm{~F}_{v} \mathrm{~S}_{1}=$ Design spectral response acceleration parameter at 1 -s period

$\mathrm{T}=$ Fundamental period of the structure, $\mathrm{s}$

$\mathrm{T}_{0}=0.2 \frac{\mathrm{S}_{D 1}}{\mathrm{~S}_{D S}}(\mathrm{~s})$

$\mathrm{T}_{\mathrm{L}}=$ Long-period transition period $(\mathrm{s})$

$\mathrm{T}_{S}=\frac{\mathrm{S}_{D 1}}{\mathrm{~S}_{D S}}(\mathrm{~s})$

$\mathrm{S}_{s}=$ Mapped MCE spectral response acceleration at short periods

$\mathrm{S}_{1}=$ Mapped MCE spectral response acceleration at 1-second period

$\mathrm{F}_{a}=$ Short-period site coefficient (at 0.2 second period)

$\mathrm{F}_{v}=$ Long-period site coefficient (at 1.0 second period)

The coefficients $\mathrm{S}_{s}, \mathrm{~S}_{1}, \mathrm{~F}_{a}$ and $\mathrm{F}_{v}$ are determined in accordance with ASCE7-10. Figure 33 shows the design response spectrum used to determine the horizontal acceleration of the models, and Table 16 presents a summary of the parameters used for this analysis. 


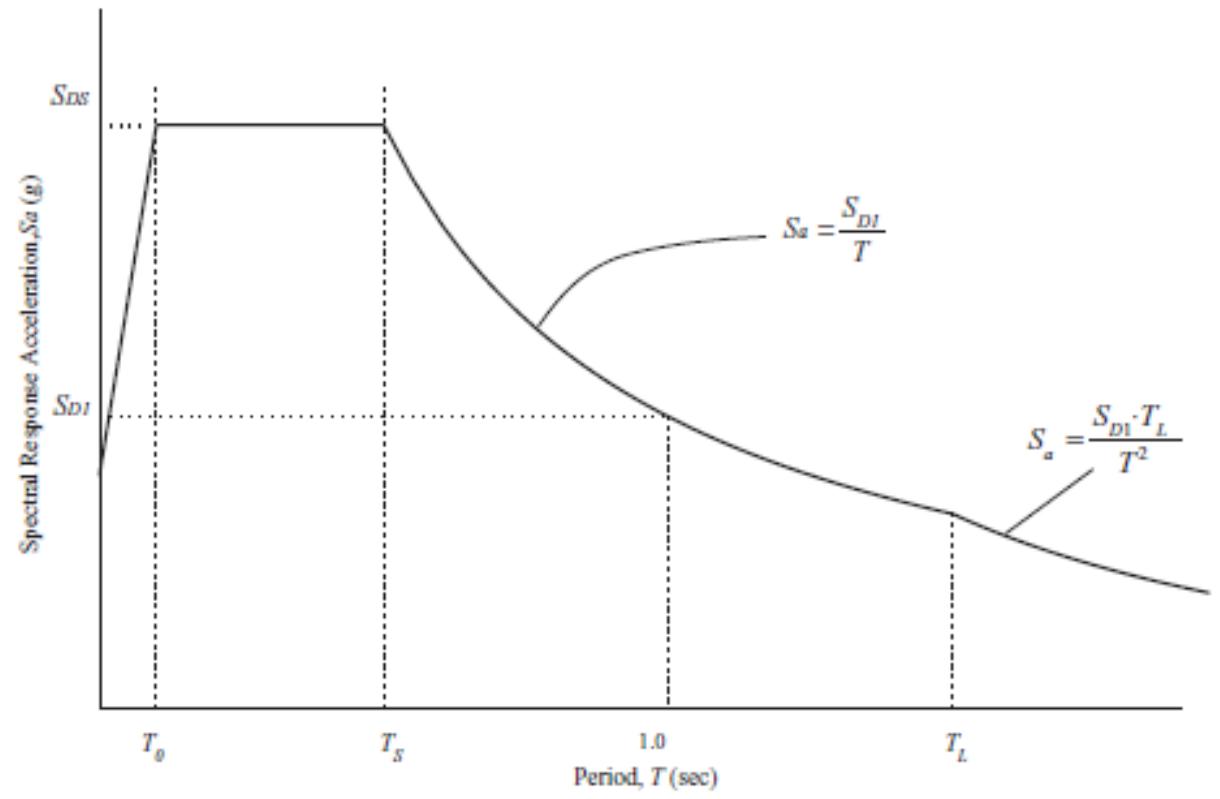

Figure 33. Design response spectrum (Adapted from ASCE7-10)

Table 16. Summary of response spectrum parameters

\begin{tabular}{|c|c|}
\hline \multicolumn{2}{|c|}{ Mapped acceleration parameters } \\
\hline $\mathrm{Ss}=$ & $2.74 \mathrm{~g}$ \\
\hline $\mathrm{S} 1=$ & $1.08 \mathrm{~g}$ \\
\hline \multicolumn{2}{|c|}{ Site coefficients } \\
\hline $\mathrm{Fa}=$ & $1.00 \mathrm{~g}$ \\
\hline $\mathrm{Fv}=$ & $1.50 \mathrm{~g}$ \\
\hline Design spectral acceleration parameters \\
\hline $\mathrm{S}_{\mathrm{DS}}=$ & $1.83 \mathrm{~g}$ (Missouri) \\
\hline $\mathrm{S}_{\mathrm{D} 1}=$ & $1.08 \mathrm{~g}$ \\
\hline \multicolumn{2}{|c|}{ Periods } \\
\hline $\mathrm{To}=$ & $0.12 \mathrm{~s}$ \\
\hline $\mathrm{Ts}=$ & $0.59 \mathrm{~s}$ \\
\hline $\mathrm{TL}=$ & $12.00 \mathrm{~s}$ \\
\hline
\end{tabular}


Presented in Figure 34 is the response spectrum used in analysis, developed in accordance with the procedure explained above.

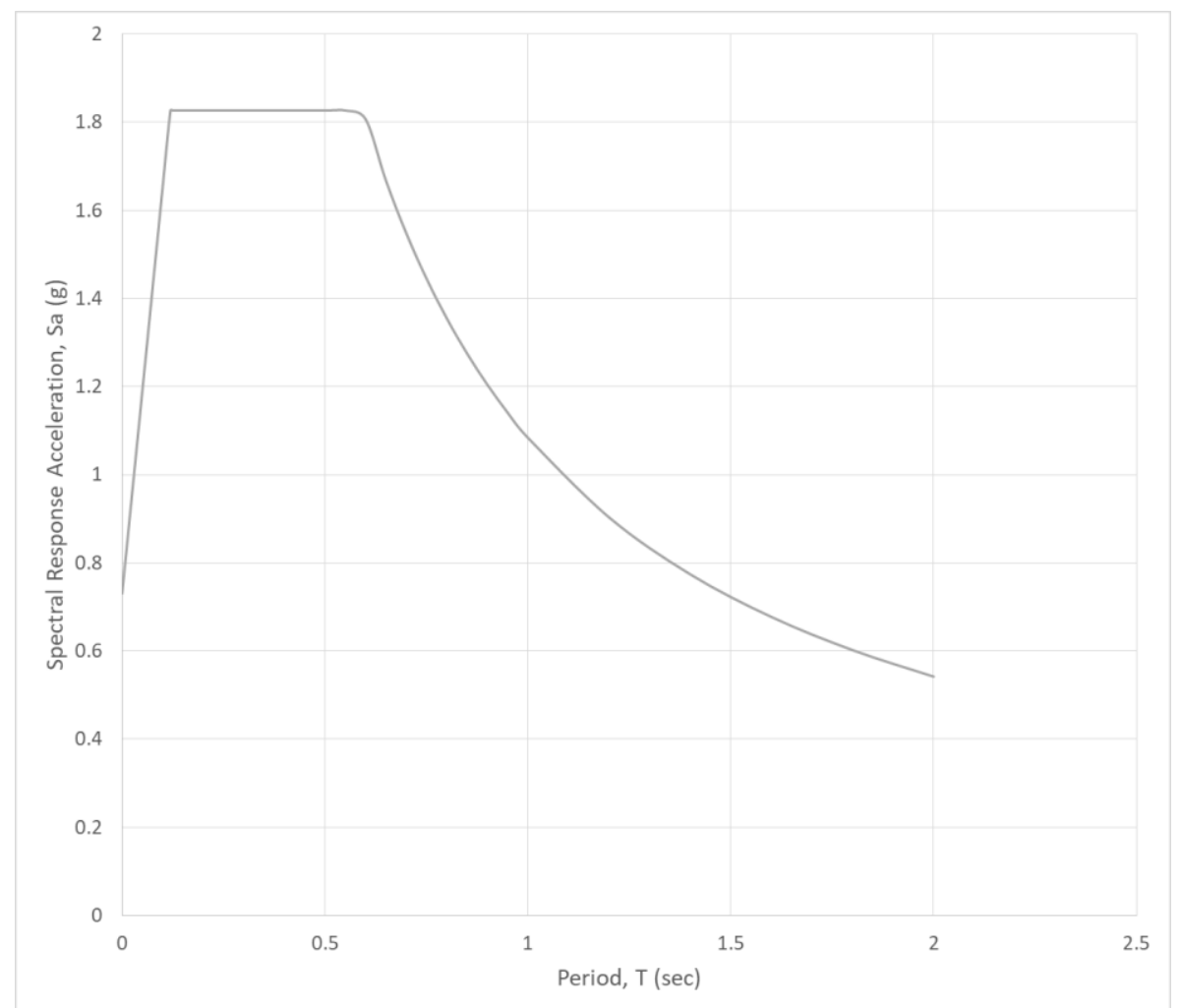

Figure 34. Response spectrum used in analysis for horizontal component of earthquake

Once the response spectrum is developed and the fundamental period is found, it is possible to determine the corresponding maximum acceleration of the studied structure. This can be done by entering into the graph (Figure 34) the calculated period and obtaining the corresponding acceleration value; equivalently, equations 44 to 47 can also be used.

For instance, the fundamental period for the first model is $0.066 \mathrm{~s}$; since it is lower than $\mathrm{T}_{0}$, equation 44 shall be used, resulting in:

$$
S_{a}=1.83\left(0.4+0.6 \frac{0.066}{0.12}\right)=1.34(g)
$$

A similar procedure is used for the other 13 models. Table 17 summarizes the results 
obtained.

Table 17. Spectral accelerations corresponding to the horizontal earthquake component

\begin{tabular}{|c|c|c|}
\hline Model & $\begin{array}{c}\text { Period } \\
(\mathrm{s})\end{array}$ & $\begin{array}{l}\text { Sa } \\
(\mathrm{g})\end{array}$ \\
\hline 1 & 0.066 & 1.34 \\
\hline 2 & 0.134 & 1.83 \\
\hline 3 & 0.062 & 1.30 \\
\hline 4 & 0.125 & 1.83 \\
\hline 5 & 0.188 & 1.83 \\
\hline 6 & 0.218 & 1.83 \\
\hline 7 & 0.098 & 1.63 \\
\hline 8 & 0.148 & 1.83 \\
\hline 9 & 0.174 & 1.83 \\
\hline 10 & 0.165 & 1.83 \\
\hline 11 & 0.206 & 1.83 \\
\hline 12 & 0.243 & 1.83 \\
\hline 13 & 0.281 & 1.83 \\
\hline 14 & 0.320 & 1.83 \\
\hline
\end{tabular}

It is noted that most of the models experience the maximum possible acceleration for the chosen site class and location; only three of them are subjected to lower values, due to the short periods associated with their response.

\subsection{Vertical component of earthquake}

In general, the design of a structure is primarily affected by the horizontal component of earthquake and there are many occasions when it comes to the design of typical structures in which the effect of vertical component is neglected since it is usually of little significance in terms of the resultant force and deflection. Despite this being often the expected behavior in most structures, for spherical shells the opposite occurs; since it is the vertical component 
that impacts the design of this type of structures most.

The most common design practice, also adopted by ACI372R-13, recommends the vertical response spectral acceleration to be two third of the horizontal acceleration of the generic Design Basis Earthquake (DBE) spectrum at the corresponding frequencies with the exception of the site specific hazard; equaling to $2 / 3 \mathrm{~S}_{\mathrm{DS}}$ in the case of this study.

On the other hand, ASCE7-10 suggests the use of a lower factor to account for the effects of vertical ground acceleration, addressing determination of the vertical response spectrum as only $20 \%$ of the horizontal response spectrum $\left(0.2 \mathrm{~S}_{\mathrm{DS}}\right)$.

As noted above, the first practice promotes a higher participation of the vertical component, making the horizontal component 1.5 times the vertical component; while for the second one, the horizontal component could be up to 5 times larger than the vertical component.

The corresponding vertical acceleration according to each practice is summarized in the following table:

Table 18. Effect of vertical earthquake component

\begin{tabular}{|c|c|}
\hline ACI 372R-13 & ASCE 7-10 \\
\hline$E_{V}=2 / 3 S_{D S}=1.22(g)$ & $E_{V}=0.2 S_{D S}=0.37(g)$ \\
\hline
\end{tabular}

\subsection{Finite element modeling}

Taking into account the aforementioned models, with the specified geometries and properties, the seismic analysis is carried out in this step.

First, an initial step is set up accounting for only the self-weight of the structure. To this effect, ABAQUS proposes an analysis module called "Static General", which is intended for such simple general cases. 
As mentioned before, the standard implicit (static) analysis is used in this study instead of the explicit dynamic analysis. In a standard implicit analysis a set of equilibrium static equations should be satisfied at each time increment before proceeding to the next. In this type of analysis, a time period for load application maintaining consistent and accurate predictions should be selected. In the same way, an amplitude for load increment should be also specified. For the purpose of this research, the load is input as a linear ramp from zero to its maximum value in a total step time of 1.0 second.

On account of the type of loading, ABAQUS offers different options for the consideration of the self-weight of the structure; since all of them are equally accurate for this type of problem, only as a matter of preference, the dead load is applied as body force in the gravity direction, as shown in Figure 35.

Once the effect of self-weight is fully taken into account; the seismic load is to be applied to the model. This requires the creation of a new step using a Static-Riks procedure, in which the initially applied conditions of the elements will be continued until one or more of the elements are going to fail (buckle) under the given conditions and as a result of continuously increasing the load.

In order to analyze the effect of the horizontal component of earthquake on the buckling response of spherical shells, it is imperative to divide the problem into two stages: first, to estimate the buckling capacity under the pure vertical seismic action; second, to analyze the buckling response of the structure under the combined effect of horizontal and vertical components, simultaneously acting on the structure.

For this purpose; in both cases, a uniformly distributed pressure equal to one is applied in $\mathrm{Y}$ direction (gravity) as a representation of the vertical earthquake excitation. While, for the case of horizontal component, a surface traction proportionally adjusted according to the Eh/Ev ratio stated previously under section 5.4 is applied in X direction. As explained before, this ratio could be different depending on the Code to be applied and the 
frequency characteristics of each model to be analyzed. A summary of the corresponding seismic loading data is shown in Table 19 for each model and Code case.

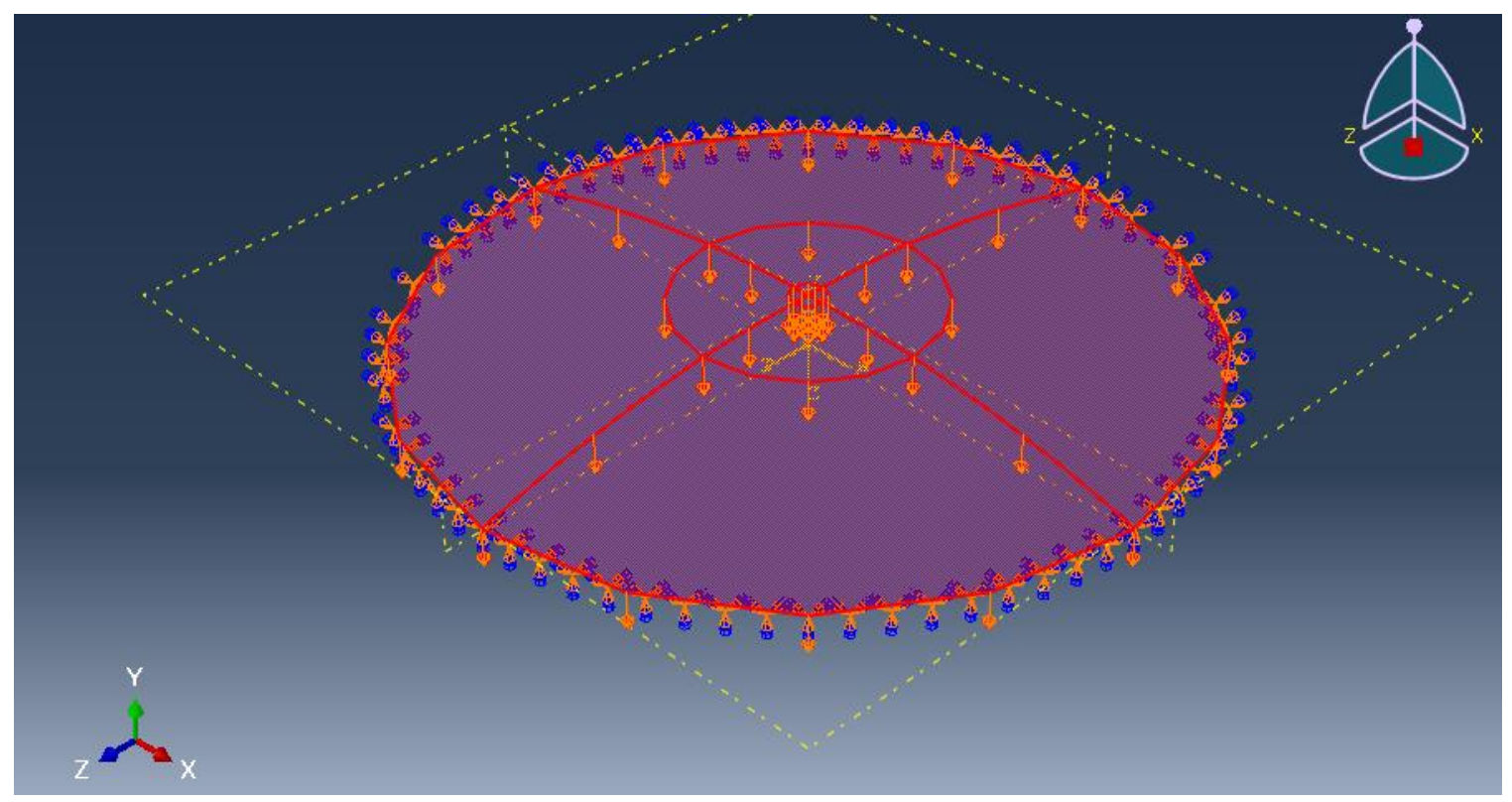

Figure 35. Self-weight loading of the dome

Table 19. Seismic loading data for FE analysis (horizontal and vertical excitation)

\begin{tabular}{|c|c|c|c|}
\hline \multirow{2}{*}{ Model } & \multirow{2}{*}{$\begin{array}{c}\text { Ev } \\
\left.\text { (kN/m }{ }^{2}\right)\end{array}$} & ASCE7-10 & ACI372R-13 \\
\cline { 3 - 4 } & & 3.675 & 1.102 \\
\hline 1 & 1.000 & 5.000 & 1.500 \\
\hline 2 & 1.000 & 3.564 & 1.069 \\
\hline 3 & 1.000 & 5.000 & 1.500 \\
\hline 4 & 1.000 & 5.000 & 1.500 \\
\hline 5 & 1.000 & 5.000 & 1.500 \\
\hline 6 & 1.000 & 4.473 & 1.342 \\
\hline 7 & 1.000 & 5.000 & 1.500 \\
\hline 8 & 1.000 & 5.000 & 1.500 \\
\hline 9 & 1.000 & 5.000 & 1.500 \\
\hline 10 & 1.000 & 5.000 & 1.500 \\
\hline 11 & 1.000 & 5.000 & 1.500 \\
\hline 12 & 1.000 & 5.000 & 1.500 \\
\hline 13 & 1.000 & 5.000 & 1.500 \\
\hline 14 & 1.000 & & \\
\hline
\end{tabular}




\subsection{Finite element results}

To evaluate the effect of the horizontal component of earthquake on the buckling failure of spherical shells, a comparative analysis between the results obtained for the two cases of pure vertical $(\mathrm{EV})$ and combined horizontal and vertical $(\mathrm{EV}+\mathrm{EH})$ seismic action is carried out.

Table 20 and Figure 36 show a summary of the results obtained under loading conditions prescribed in ACI372R-13. A consistent trend can be observed in the obtained results, as including the horizontal loading has resulted in a reduction in the buckling capacity of the models in all cases. However, such effect is quite negligible, not more than $0.50 \%$ assuming the ACI372R-13 seismic loading scenario.

Table 20. Buckling pressure from FEA under seismic action (ACI372R-13)

\begin{tabular}{|c|c|c|c|}
\hline \multirow{2}{*}{ Model } & $\begin{array}{c}\text { Buckling Pressure } \\
\text { EV } \\
(\mathrm{kPa})\end{array}$ & $\begin{array}{c}\text { Buckling Pressure } \\
\text { EV+EH } \\
(\mathrm{kPa})\end{array}$ & $\begin{array}{c}\text { Error } \\
(\%)\end{array}$ \\
\hline 1 & 64.65 & 64.54 & $-0.18 \%$ \\
\hline 2 & 16.18 & 16.17 & $-0.10 \%$ \\
\hline 3 & 74.21 & 74.10 & $-0.16 \%$ \\
\hline 4 & 18.68 & 18.65 & $-0.14 \%$ \\
\hline 5 & 14.96 & 14.93 & $-0.19 \%$ \\
\hline 6 & 10.96 & 10.94 & $-0.18 \%$ \\
\hline 7 & 54.86 & 54.65 & $-0.37 \%$ \\
\hline 8 & 18.78 & 18.77 & $-0.08 \%$ \\
\hline 9 & 15.84 & 15.78 & $-0.37 \%$ \\
\hline 10 & 18.06 & 18.02 & $-0.21 \%$ \\
\hline 11 & 16.61 & 16.58 & $-0.18 \%$ \\
\hline 12 & 20.27 & 20.20 & $-0.35 \%$ \\
\hline 13 & 25.96 & 25.86 & $-0.40 \%$ \\
\hline 14 & 30.83 & 30.70 & $-0.42 \%$ \\
\hline
\end{tabular}



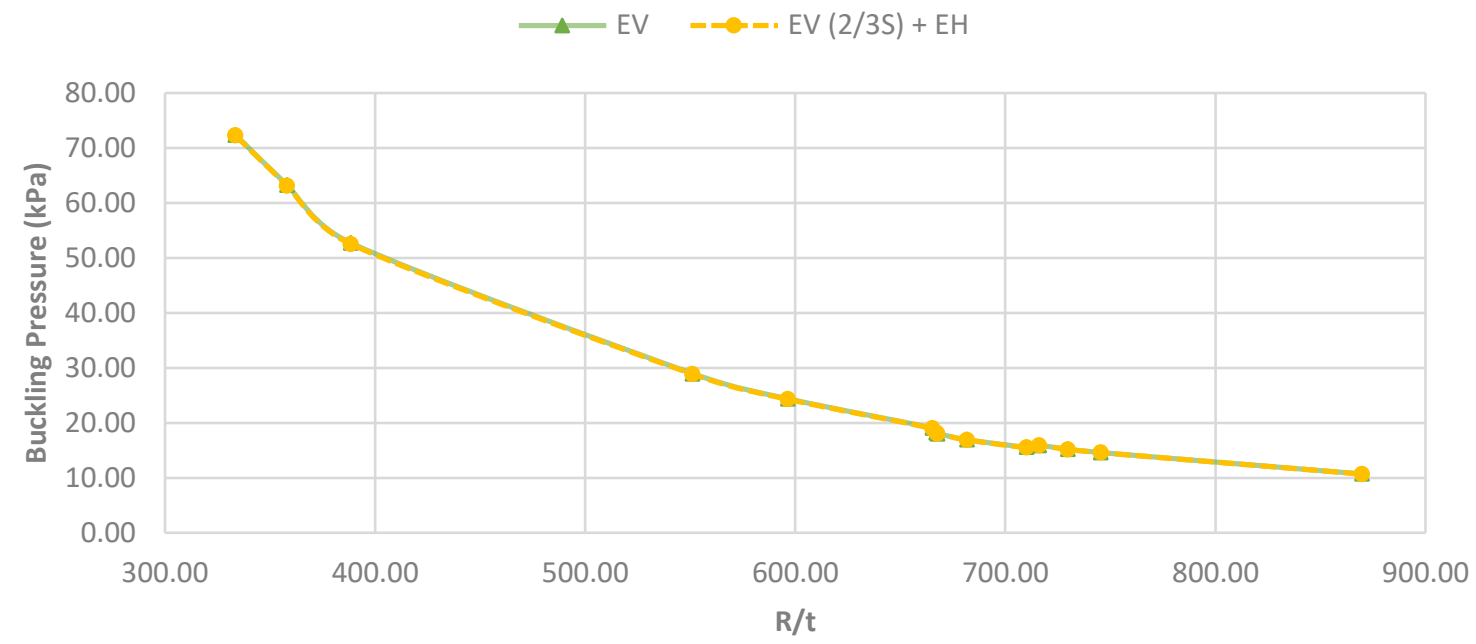

Figure 36. Buckling pressure from FEA under seismic action (ACI372R-13)

For the case of seismic loading condition in accordance with ASCE7-10, the results are given in Table 21 and Figure 37. A larger reduction in the results due to the inclusion of horizontal component is evident in this case, reaching a change of as much as $4 \%$ in buckling failure capacity of the domes.

In the same vein, it can be observed from Figure 37 that the contribution of horizontal component of earthquake in the buckling capacity of the domes is more noticeable in models with lower aspect ratios R/t (left upper part of the graph) as compared to those with larger $\mathrm{R} / \mathrm{t}$ (right lower part of the graph).

In order to make a more in depth analysis of the results and with the intention of determining the key factor affecting the buckling capacity of the models, the results produced assuming ASCE loading criteria are sorted in descending order with respect to the error percentage calculated as shown in Table 22. 
Table 21. Buckling pressure from FEA under seismic action (ASCE7-10)

\begin{tabular}{|c|c|c|c|}
\hline \multirow{3}{*}{ Model } & $\begin{array}{c}\text { Buckling Pressure } \\
\text { EV } \\
(\mathrm{kPa})\end{array}$ & $\begin{array}{c}\text { Buckling Pressure } \\
\text { EV+EH } \\
(\mathrm{kPa})\end{array}$ & Error \\
& 64.65 & 63.40 & $(\%)$ \\
\hline 1 & 16.18 & 15.83 & $-1.97 \%$ \\
\hline 2 & 74.21 & 72.95 & $-2.26 \%$ \\
\hline 3 & 18.68 & 18.20 & $-1.74 \%$ \\
\hline 4 & 14.96 & 14.65 & $-2.63 \%$ \\
\hline 5 & 10.96 & 10.79 & $-2.08 \%$ \\
\hline 6 & 54.86 & 52.57 & $-1.59 \%$ \\
\hline 7 & 18.78 & 18.35 & $-4.36 \%$ \\
\hline 8 & 15.84 & 15.50 & $-2.38 \%$ \\
\hline 9 & 18.06 & 17.65 & $-2.19 \%$ \\
\hline 10 & 16.61 & 16.24 & $-2.29 \%$ \\
\hline 11 & 20.27 & 19.80 & $-2.26 \%$ \\
\hline 12 & 25.96 & 25.27 & $-2.39 \%$ \\
\hline 13 & 30.83 & 29.94 & $-2.74 \%$ \\
\hline 14 & & & $-2.97 \%$ \\
\hline
\end{tabular}

$\simeq \quad \mathrm{EV} \quad \ldots \in \mathrm{EV}(0.2 \mathrm{~S})+\mathrm{EH}$

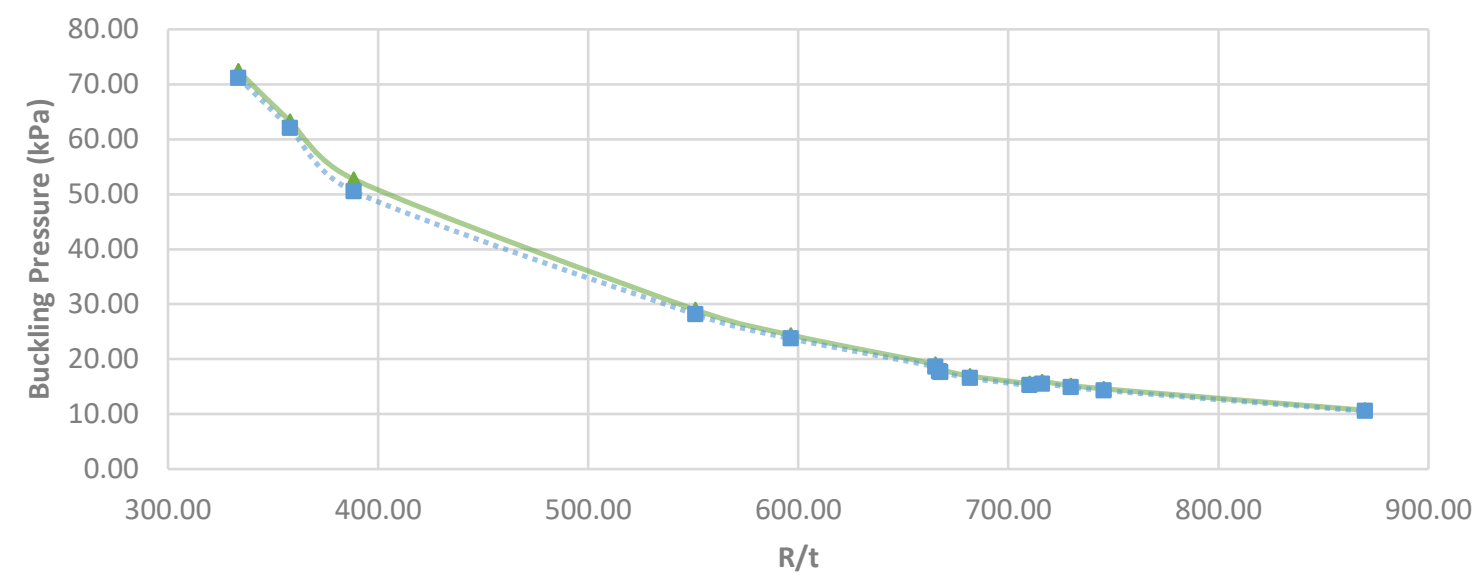

Figure 37. Buckling pressure from FEA under seismic action (ASCE7-10) 
Table 22. Models sorted by error percentage

\begin{tabular}{|c|c|c|c|}
\hline Model & R/t & Span : Rise & $\begin{array}{c}\text { Error (\%) } \\
\text { EV vs. EV+EH }\end{array}$ \\
\hline 7 & 388 & 10 & $4.36 \%$ \\
\hline 14 & 551 & 8 & $2.97 \%$ \\
\hline 13 & 597 & 8 & $2.74 \%$ \\
\hline 4 & 667 & 13 & $2.63 \%$ \\
\hline 12 & 665 & 8 & $2.39 \%$ \\
\hline 8 & 668 & 10 & $2.38 \%$ \\
\hline 10 & 682 & 8 & $2.29 \%$ \\
\hline 11 & 710 & 8 & $2.26 \%$ \\
\hline 2 & 716 & 14 & $2.26 \%$ \\
\hline 9 & 730 & 10 & $2.19 \%$ \\
\hline 5 & 746 & 13 & $2.08 \%$ \\
\hline 1 & 358 & 14 & $1.97 \%$ \\
\hline 3 & 334 & 13 & $1.74 \%$ \\
\hline 6 & 870 & 13 & $1.59 \%$ \\
\hline
\end{tabular}

The above results suggest that the reducing effect of the horizontal component on buckling capacity is higher for the cases with lower radius-to-thickness ratios; consequently, as the ratio increases the difference between the two sets of data becomes smaller. Regarding the effect of the span-to-rise ratio, no clear trend can be identified; however, as a general tendency, it can be stated that lower span to rise ratios could potentially result in higher errors.

As an exception to the above general rule, one can notice that despite having the two lowest radius-to-thickness (R/t) ratios, Models 1 and 3 are not highly influenced by the effect of horizontal component on their buckling capacity, as expected. This can be justified by the fact that these models have at the same time the two highest span-to-rise ratios, suggesting that this factor can be as much effective as $\mathrm{R} / \mathrm{t}$.

In general, in all considered cases, the effect of the horizontal component is noticeable in the measured FE results; however, it is important to emphasize that the deviation between the buckling capacity values calculated with and without this component becomes greater when higher ratios of $\mathrm{EH} / \mathrm{EV}$ are considered, otherwise, the change in the 
results is almost imperceptible.

\subsection{Comparison of FE results with current practice}

In order to determine the accuracy of current practice in predicting the buckling failure of spherical shell structures, the results previously obtained are now compared with the analytical results proposed by ACI.

Table 23 and Figure 38 show the comparison of results between FEA and ACI obtained under the pure vertical seismic action. It can be noted from the table that the average error estimated is found to be around $0.60 \%$ for all models with the exception of the last 3 models where two layers of rebar are incorporated, resulting in considerably higher average error of $10.20 \%$.

It is noted that in some of the models with higher span-to-rise ratios, the FE results are lower than those of ACI leading to an unconservative design; however, this difference is negligible, and is not considered a risk to the structural safety when it comes to design.

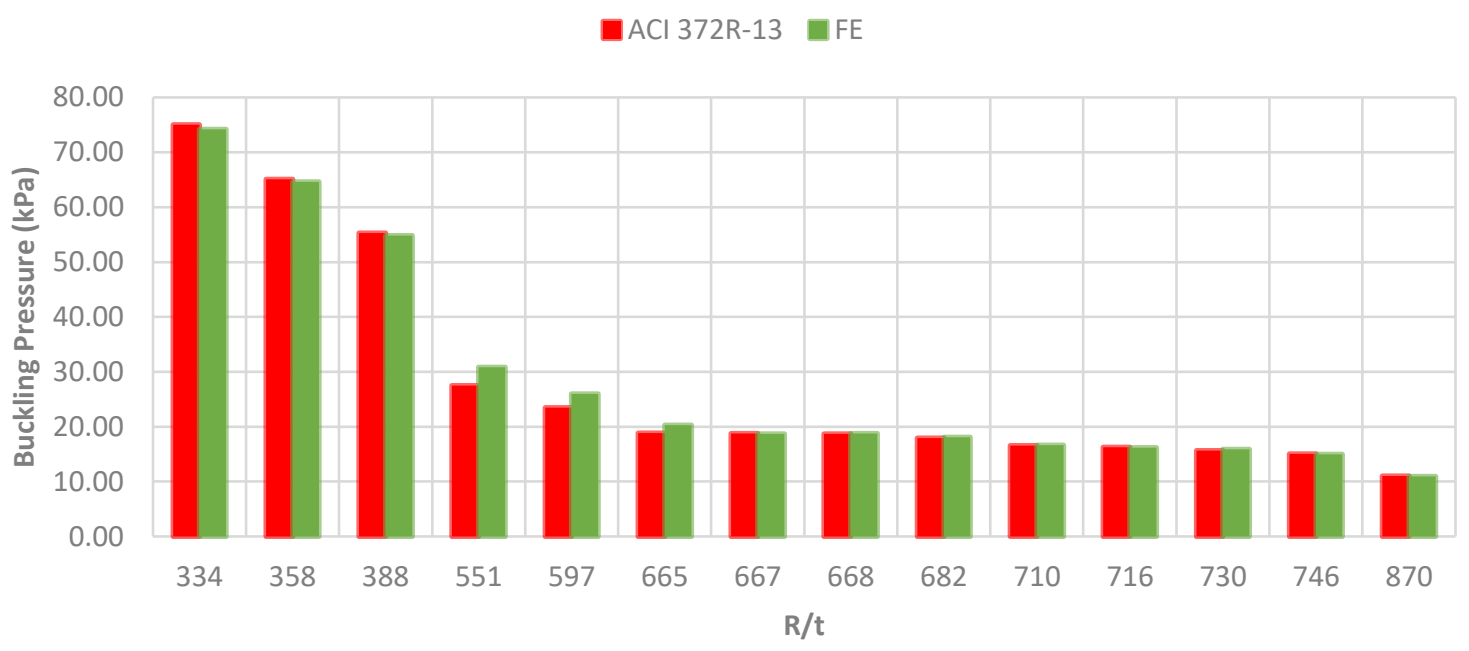

Figure 38. ACI vs. FE results under pure vertical seismic action 
Table 23. ACI vs. FE results under pure vertical seismic action

\begin{tabular}{|c|c|c|c|c|c|}
\hline Model & R/t & Span : Rise & $\begin{array}{c}\text { Buckling Pressure } \\
\text { ACI } \\
(\mathrm{kPa})\end{array}$ & $\begin{array}{c}\text { Buckling Pressure } \\
\text { FE } \\
(\mathrm{kPa})\end{array}$ & $\begin{array}{c}\text { Error } \\
(\%)\end{array}$ \\
\hline 1 & 358 & 14 & 65.04 & 64.65 & $0.60 \%$ \\
\hline 2 & 716 & 14 & 16.27 & 16.18 & $0.53 \%$ \\
\hline 3 & 334 & 13 & 74.99 & 74.21 & $1.03 \%$ \\
\hline 4 & 667 & 13 & 18.75 & 18.68 & $0.37 \%$ \\
\hline 5 & 746 & 13 & 15.01 & 14.96 & $0.36 \%$ \\
\hline 6 & 870 & 13 & 11.03 & 10.96 & $0.66 \%$ \\
\hline 7 & 388 & 10 & 55.30 & 54.86 & $0.80 \%$ \\
\hline 8 & 668 & 10 & 18.70 & 18.78 & $-0.44 \%$ \\
\hline 9 & 730 & 10 & 15.66 & 15.84 & $-1.17 \%$ \\
\hline 10 & 682 & 8 & 17.95 & 18.06 & $-0.60 \%$ \\
\hline 11 & 710 & 8 & 16.54 & 16.61 & $-0.44 \%$ \\
\hline 12 & 665 & 8 & 18.84 & 20.27 & $-7.58 \%$ \\
\hline 13 & 597 & 8 & 23.44 & 25.96 & $-10.75 \%$ \\
\hline 14 & 551 & 8 & 27.46 & 30.83 & $-12.27 \%$ \\
\hline
\end{tabular}

A similar analysis is carried out under the combined action of vertical and horizontal earthquake components; assuming both ACI372R-13 and ASCE7-10 criteria.

From Table 24 and Figure 39, it can be observed that in the case of the first loading condition (ACI372R-13), in general the difference between analytical and numerical results is slightly lower under the combined action than what obtained previously under the pure vertical action; measuring an average difference of around $0.66 \%$ without taking into account the last three models. For the last three models alone an average difference of $9.70 \%$ is obtained. 
Table 24. ACI vs. FE results under combined seismic action (seismic loading as per ACI372R-13)

\begin{tabular}{|c|c|c|c|}
\hline \multirow{3}{*}{ Model } & $\begin{array}{c}\text { Buckling Pressure } \\
\text { ACI } \\
(\mathrm{kPa})\end{array}$ & $\begin{array}{c}\text { Buckling Pressure } \\
\text { FE } \\
(\mathrm{kPa})\end{array}$ & Error \\
& 65.04 & 64.54 & $0.78 \%$ \\
\hline 1 & 16.27 & 16.17 & $0.63 \%$ \\
\hline 2 & 74.99 & 74.10 & $1.19 \%$ \\
\hline 3 & 18.75 & 18.65 & $0.51 \%$ \\
\hline 4 & 15.01 & 14.93 & $0.55 \%$ \\
\hline 5 & 11.03 & 10.94 & $0.84 \%$ \\
\hline 6 & 55.30 & 54.65 & $1.17 \%$ \\
\hline 7 & 18.70 & 18.77 & $-0.37 \%$ \\
\hline 8 & 15.66 & 15.78 & $-0.80 \%$ \\
\hline 9 & 17.95 & 18.02 & $-0.39 \%$ \\
\hline 10 & 16.54 & 16.58 & $-0.26 \%$ \\
\hline 11 & 18.84 & 20.20 & $-7.21 \%$ \\
\hline 12 & 23.44 & 25.86 & $-10.31 \%$ \\
\hline 13 & 27.46 & 30.70 & $-11.80 \%$ \\
\hline 14 & & & \\
\hline
\end{tabular}

The results given in Table 25 and Figure 40 show that assuming the second loading condition (ASCE7-10), the results obtained from FEA for all the models with a single layer of rebar are lower than the Code estimations, reaching difference percentages of up to $5 \%$ in some cases. Such trend indicates that considering ASCE7-10 seismic loading condition can lead to an overestimation of buckling capacity by the Code which can in turn yield an unconservative design of such structures under combined seismic effects. However, since the error is still fairly small (not more than 5\%), the Code safety factors considered as a compromise between precision and simplicity are deemed enough to compensate for this shortfall.

On the other hand, it can be noticed that the difference between FE and ACI results in the case of last three models is reduced as a consequence of the addition of the horizontal component of earthquake. Furthermore, for these three models the buckling capacity estimated numerically is higher than the ACI proposed value, in turn leading to conservative results. 


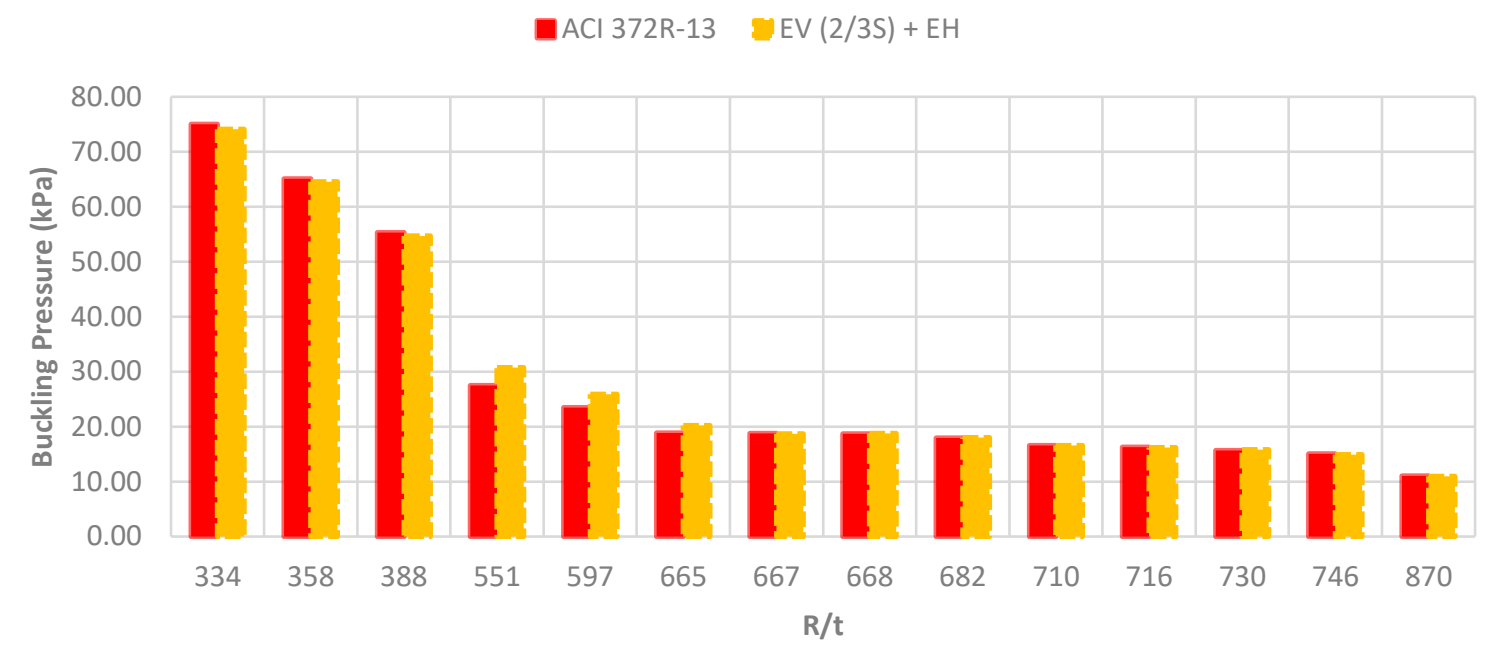

Figure 39. ACI vs. FE results under combined seismic action (seismic loading as per ACI372R-13)

Table 25. ACI vs. FE results under combined seismic action (seismic loading as per ASCE7-10)

\begin{tabular}{|c|c|c|c|c|c|}
\hline Model & $\mathrm{R} / \mathrm{t}$ & Span : Rise & $\begin{array}{c}\text { Buckling Pressure } \\
\text { ACI } \\
(\mathrm{kPa})\end{array}$ & $\begin{array}{c}\text { Buckling Pressure } \\
\text { FE } \\
(\mathrm{kPa})\end{array}$ & Error \\
\hline 1 & 358 & 14 & 65.04 & 63.40 & $2.52 \%$ \\
\hline 2 & 716 & 14 & 16.27 & 15.83 & $2.73 \%$ \\
\hline 3 & 334 & 13 & 74.99 & 72.95 & $2.72 \%$ \\
\hline 4 & 667 & 13 & 18.75 & 18.20 & $2.92 \%$ \\
\hline 5 & 746 & 13 & 15.01 & 14.65 & $2.39 \%$ \\
\hline 6 & 870 & 13 & 11.03 & 10.79 & $2.21 \%$ \\
\hline 7 & 388 & 10 & 55.30 & 52.57 & $4.94 \%$ \\
\hline 8 & 668 & 10 & 18.70 & 18.35 & $1.89 \%$ \\
\hline 9 & 730 & 10 & 15.66 & 15.50 & $0.99 \%$ \\
\hline 10 & 682 & 8 & 17.95 & 17.65 & $1.65 \%$ \\
\hline 11 & 710 & 8 & 16.54 & 16.24 & $1.78 \%$ \\
\hline 12 & 665 & 8 & 18.84 & 25.80 & $-5.07 \%$ \\
\hline 13 & 597 & 8 & 23.44 & 29.94 & $-7.80 \%$ \\
\hline 14 & 551 & 8 & 27.46 & $-9.04 \%$ \\
\hline
\end{tabular}




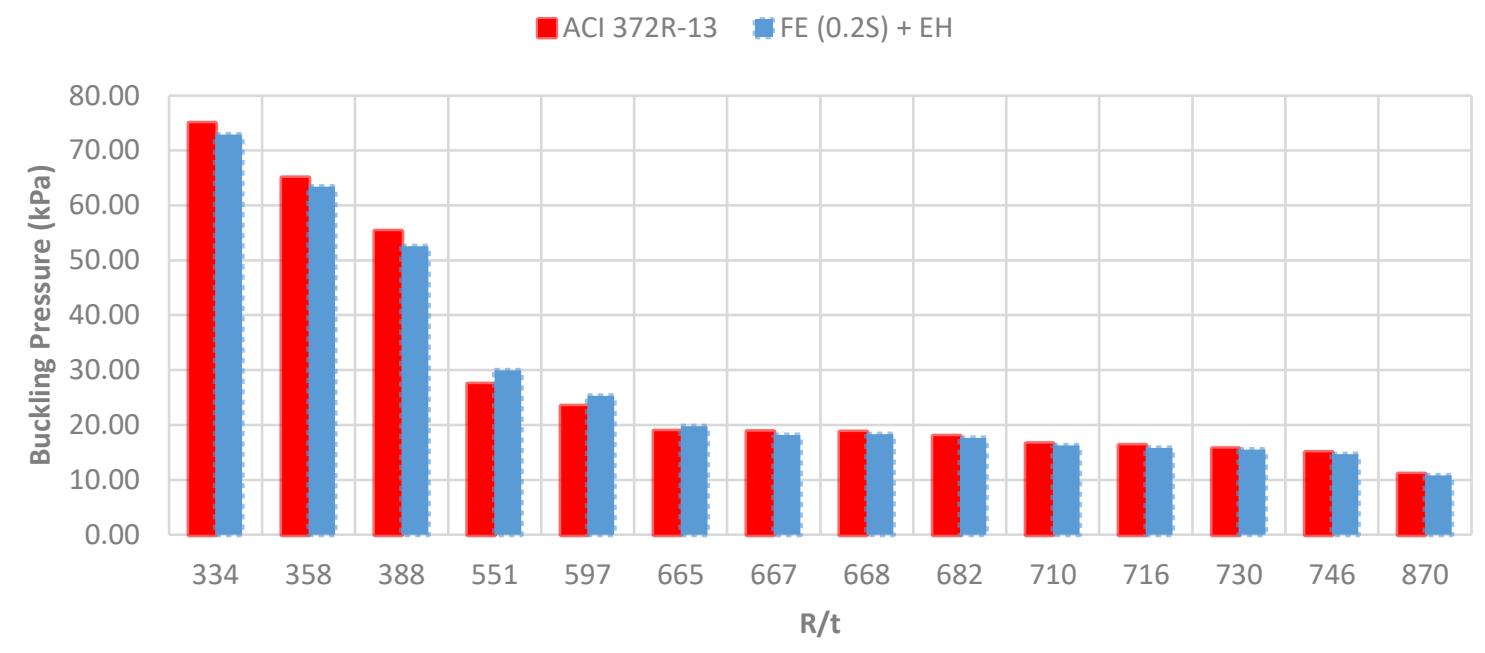

Figure 40. ACI vs. FE results under combined seismic action (seismic loading as per ASCE7-10)

\section{$5.8 \quad$ Summary}

In this chapter, the main objective was to determine the effect of the horizontal seismic component on the critical buckling pressure of spherical shells; however, prior to this, some procedures, in addition to the previously mentioned finite element technique, had to be introduced to obtain and apply this type of loading on the structure.

One of these new techniques corresponded to a free vibration analysis; this was performed in ABAQUS in order to obtain the fundamental frequency of each model, necessary for the estimation of the earthquake magnitude. Using this method, the fundamental natural frequency was found.

The next step, corresponded to the determination of the response spectrum as proposed by ASCE7-10; to this effect, one of the highest seismic zones in the US was selected in accordance with the national seismic hazard mapping project website, corresponding to the geographic coordinates of Missouri; this along with some derived 
factors, made the creation of the response spectrum possible. This in conjunction with the fundamental frequencies previously found, allowed the determination of the corresponding horizontal spectral acceleration for each model.

While for the case of the vertical component, two design methods were used; the one proposed by $\mathrm{ACI}$, corresponding to $2 / 3 \mathrm{~S}_{\mathrm{ds}}$ and the other suggested by $\mathrm{ASCE}$, equal to $0.2 \mathrm{~S}_{\mathrm{ds}}$. The use of these two different criteria, results in two different Eh/Ev ratios, establishing, therefore, different levels of participation of horizontal component; a factor that was considered later in the analysis.

These two seismic components, were later applied to the structure as static loading; however, as previously explained, since the Static Riks procedure was used, these loads kept increasing in accordance with the Load Proportionality Factor (LPF) until the maximum capacity of the structure was reached. Finally, the critical buckling pressure was found as the maximum pressure before the sudden failure of the structure. The results were then computed for two specific cases: first, under the pure vertical component, and second under the combined effect of horizontal and vertical components.

Comparing the results showed that the inclusion of the horizontal component always led to lower buckling capacities. On the one hand, in the cases where ACI loading condition was used, the maximum error found was only $0.42 \%$. While in the cases where ASCE loading criterion was applied, a higher difference was shown, resulting in error percentages in the range of $1.59 \%$ to $4.36 \%$. The results further demonstrated that the higher the participation of the horizontal component, the lower the buckling capacity of the dome.

When organizing the results in accordance with the error obtained, it was observed that the effect of the horizontal component was also influenced by the radius-to-thickness ratio used. Generally speaking, lower ratios led to higher differences, and therefore, lower buckling pressures. In summary, the buckling capacity of a spherical shell is always reduced due to the effect of the horizontal earthquake component; however, the magnitude of this 
reduction is highly influenced by the level of participation of this component and the geometrical configuration of the structure.

Finally, the FE results previously obtained were later compared with the ACI analytical solution; revealing that ACI is overestimating the buckling capacity of the structure in most of the cases, reaching a 4.94\% difference when ASCE earthquake loading criterion is considered. While in the cases where the vertical component is measured according to ACI372R-13, this difference decreases to a maximum of 1.19\%, making ACI in some cases a more conservative approach.

On another matter, once again placing the reinforcement in two layers instead of one is shown to have a significant effect on the buckling capacity of the structure. In the last three models with two layers of reinforcement, despite noting some reduction in the buckling capacity due to the effect of horizontal component, the results proposed by ACI Code are still on the conservative side when comparing with the numerical ones. 


\section{CHAPTER 6}

\section{SUMMARY, CONCLUSIONS AND RECOMMENDATIONS}

\subsection{Summary}

The main objective of this investigation was to analyze the effect of the horizontal component of earthquake on the buckling failure of concrete spherical shells. To this effect, a deep understanding of the behavior of shells, as well as the theories that predict their failure were required in advance.

According to the previous investigations; due to the geometry of the spherical shells, the type of failure that these structures experience is an instability failure and not a material one; therefore, their behavior will be controlled by their buckling capacity. To this effect, several formulations were proposed to predict this failure; the most commonly known is the "Classical buckling equation" proposed by Timoshenko. However, this formulation was limited to a linear analysis, which resulted in a type of failure commonly known as "bifurcation buckling”.

Further investigations proved that this type of buckling was only achieved if the structure was geometrically perfect and where the effect of edge condition was ignored; which is not usually a real case scenario. Therefore, Zarghamee and Heger (1983), later proposed a formulation that considered a more realistic solution, and closely predicted the snap-through buckling of spherical shells. Considering its accuracy, ACI adopted the

formulation for the roofing design of Circular Wire and Strand-Wrapped Prestressed Concrete Structures in ACI373R-13 code. They adjusted this formulation, in order to include the effect of the vertical component of earthquake; however, as observed in the equation the horizontal component was neglected from the design.

For this reason and given that little or no information is found with respect to this effect, the aim of this investigation is to analyze the effect of the horizontal seismic excitation 
on dome structures, in order to provide a better understanding of this effect and more available data for future designs.

To analyze the buckling behavior of such a complex problem, a numerical technique was first developed to analyze simple cases; and once its accuracy was verified, the procedure was applied in the analysis of more complicated cases. In this vein, the finite element software ABAQUS was selected, using which both linear and nonlinear buckling analyses were made possible. For the first one, an eigenvalue bifurcation routine was implemented; while for the second case, a static Riks substep was employed.

After obtaining the buckling pressure for each case, the results were compared with the corresponding analytical formulation. The comparison showed that the developed finite element technique was quite accurate, since the numerical results were in good agreement with those obtained from the analytical solution.

However, in the cases where two layers of rebar were used, greater differences between the numerical and the analytical formulation were constantly found, since the analytical formulation does not take that into account this condition.

Once the accuracy of the proposed finite element technique was verified, the models were analyzed under the seismic loading effects. In this manner, a free vibration analysis was performed in order to obtain the natural frequency of the structure. Using this and the response spectrum from ASCE, the corresponding horizontal acceleration for each model was found.

While, for the vertical component of earthquake, the formulations proposed by ACI 372R-13 and ASCE 7-10 were used, as a result of which two levels of relative participation for the horizontal component of earthquake were obtained.

Initially, the structure was analyzed under the effect of the pure vertical loading; once the buckling failure was obtained, the horizontal component was introduced into the analysis 
and the results for the two cases were compared.

These results showed that despite the level of participation of the horizontal component of earthquake, a reduction in the buckling capacity of the structure was constantly found when comparing with the results under pure vertical loading.

Finally, the buckling pressure obtained from the numerical analysis was compared with ACI solution and was shown that in most cases the Code lightly overestimated the buckling capacity of the structure.

\subsection{Conclusions}

In this study the effect of the horizontal component of earthquake on the buckling response of spherical shells was investigated. Based on the results obtained, the following conclusions are made:

1. The proposed FE method provides a good agreement with the analytical results in both linear and nonlinear cases. Therefore, this method could be used in future investigations.

2. Comparing the snap-trough buckling (nonlinear analysis) with the bifurcation buckling (linear analysis), a reduction of around $72 \%$ in the buckling capacity is always found; in agreement with the conclusions of previous research.

3. In accordance with previous investigations, an imperfection with a shallowness parameter of four results in the most critical buckling case scenario.

4. Generally the buckling capacity of spherical shells fixed at the base are lower compared to those that are pinned; however, this is not true in all cases. The obtained difference between the two cases is negligible suggesting that such effect is not of much significance. 
5. Including two layers of rebar result in a significant increase in the buckling pressure. Although the results obtained from the ACI formulation are on conservative side, it is not accurate in the estimation of buckling capacity of double layered reinforced concrete domes.

6. Considering the combined effect of the horizontal and vertical components of earthquake, it is concluded that including the horizontal component leads to a reduction in the buckling capacity; however, the impact of this reduction is highly influenced by the Eh/Ev ratio assumed.

7. ACI372R-13 and ASCE7-10 propose different formulations for considering the contribution of vertical earthquake forces, the first one results in lower $\mathrm{Eh} / \mathrm{Ev}$ in comparison with the second one. The results obtained under both conditions consistently indicate that there is always a reduction in the buckling pressure; however, this reduction only becomes significant when relatively higher $\mathrm{Eh} / \mathrm{Ev}$ ratios are considered; as is the case in ASCE7-10.

8. The effect of the horizontal component of earthquake on the buckling capacity of the dome structure increases as the radius-to-thickness ratios decreases; a similar behavior is also observed for low dome rise/base diameter ratios; however, since in the case of this study they are linked in most of the cases to low radius to thickness ratios, no conclusive finding is obtained with regards to the effect of this parameter.

9. According to the cases studied and the results obtained, the inclusion of the horizontal component of earthquake could lead to a reduction of up to $4.36 \%$ in the buckling capacity of the structure.

10. In general, ACI372R-13 provides a good estimation of the buckling pressure of spherical shells; however, it is observed that the results obtained by this formulation are slightly overestimating the buckling capacity, especially in the cases where the 
effect of horizontal component of earthquake is taken into account, in which a maximum difference of $4.94 \%$ is obtained.

\subsection{Recommendations for future studies}

Based on this research study, some suggestions for further research on the effect of the horizontal component of earthquake on the buckling of spherical shells are presented below:

1. The dynamic response of spherical shells under the combined effect of vertical and horizontal acceleration can be further studied, in order to resemble as much as possible the reality of the seismic forces.

2. The effect of including the wall and its connections to the roof can be investigated in further researches. Different types of fixities can be studied as well.

3. The effect of material nonlinearity including reinforcement yielding, concrete cracking and crushing as well as creep in concrete can be incorporated in future investigations. Even though, the buckling is expected to happen at nominal stresses far below yielding, it is known that the creep could have a substantial effect on the buckling behavior.

4. The fabrication and testing of small samples is suggested to be analyzed in a future research in order to support the numerical results obtained. 


\section{APPENDIX A: COMPARISON BETWEEN FIXED AND HINGED BOUNDARY CONDITIONS}

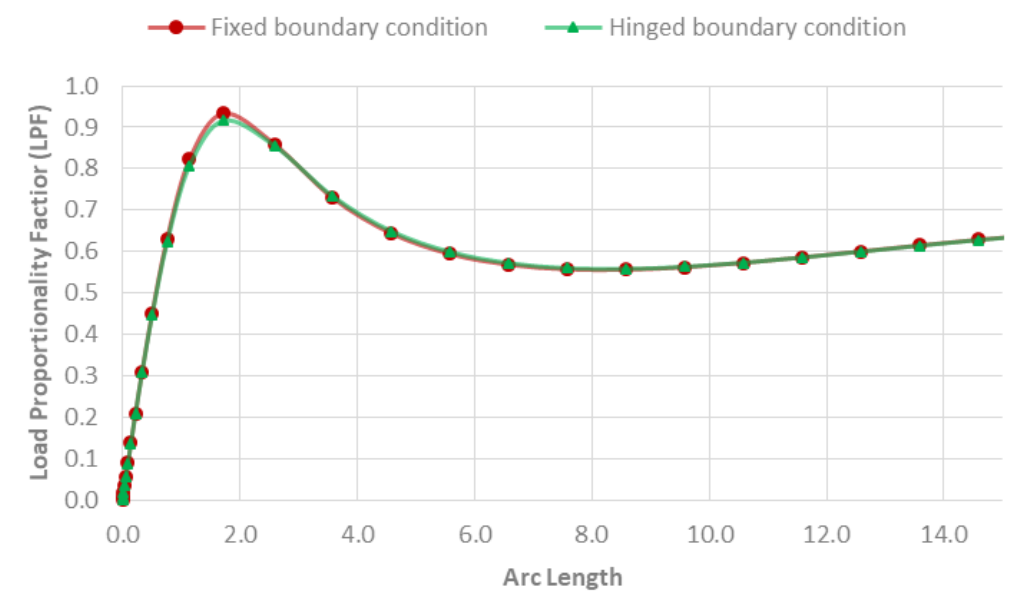

Figure A. 1 Comparison of boundary conditions - Model 1

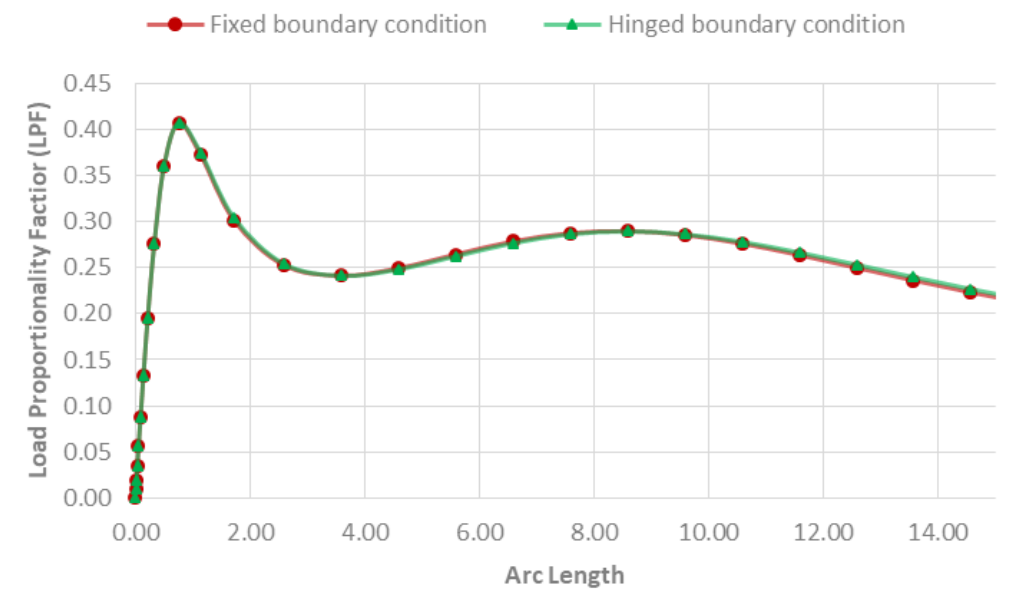

Figure A. 2 Comparison of boundary conditions - Model 2 


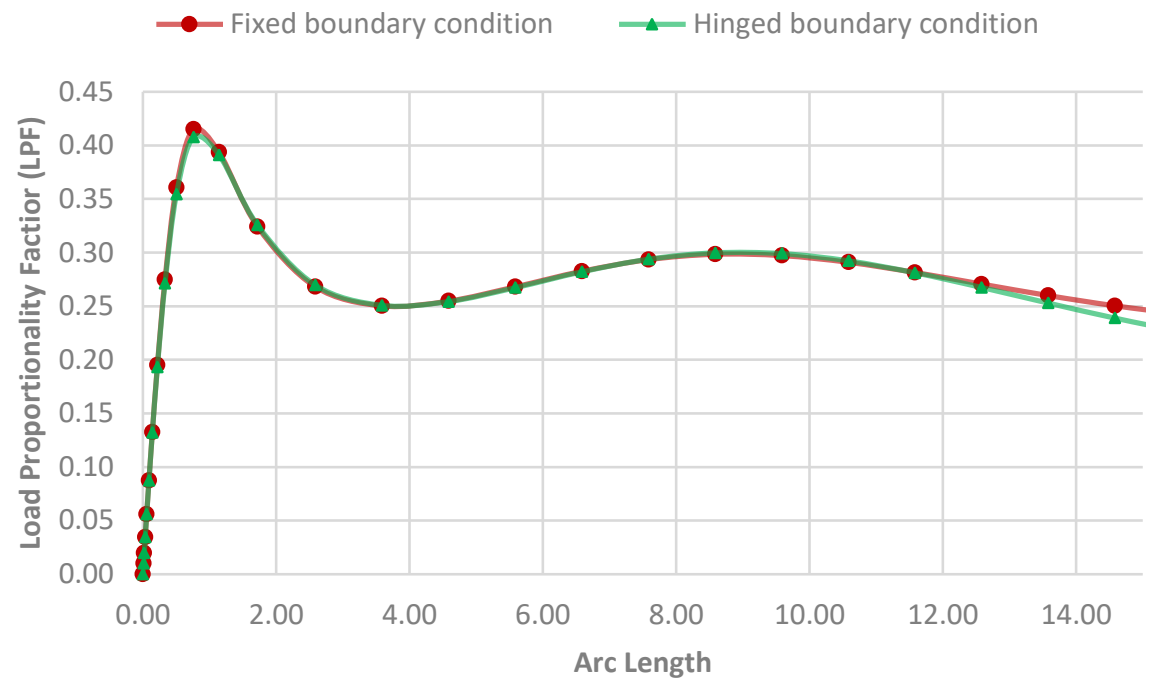

Figure A. 3 Comparison of boundary conditions - Model 3

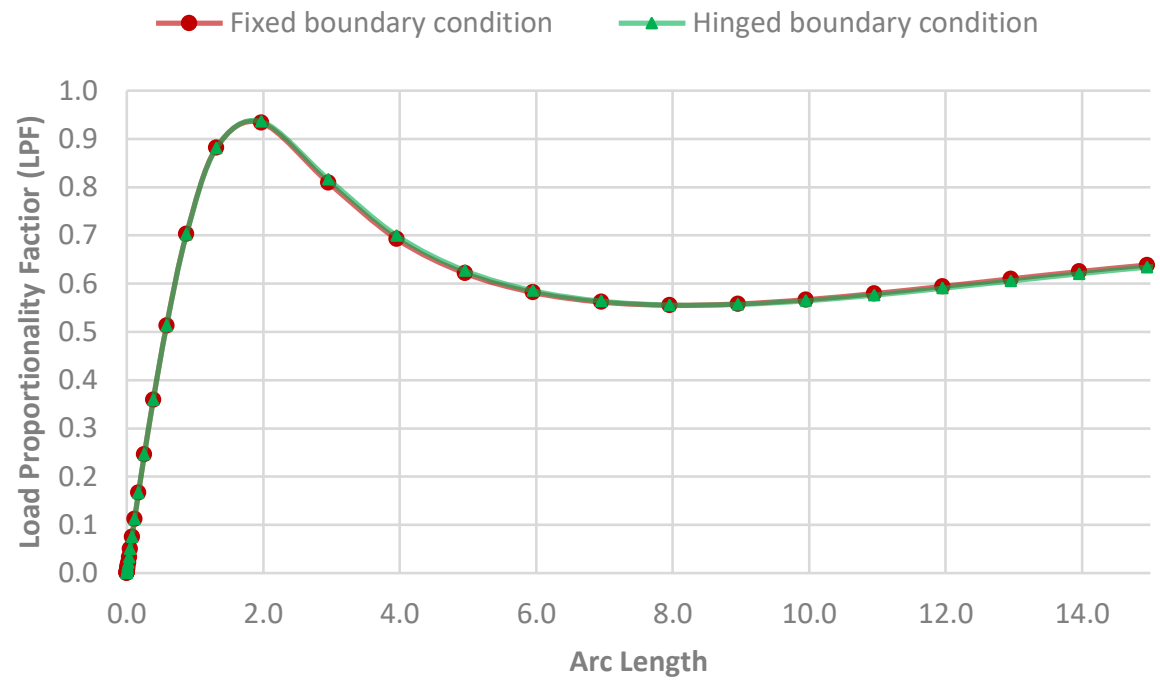

Figure A. 4 Comparison of boundary conditions - Model 4 


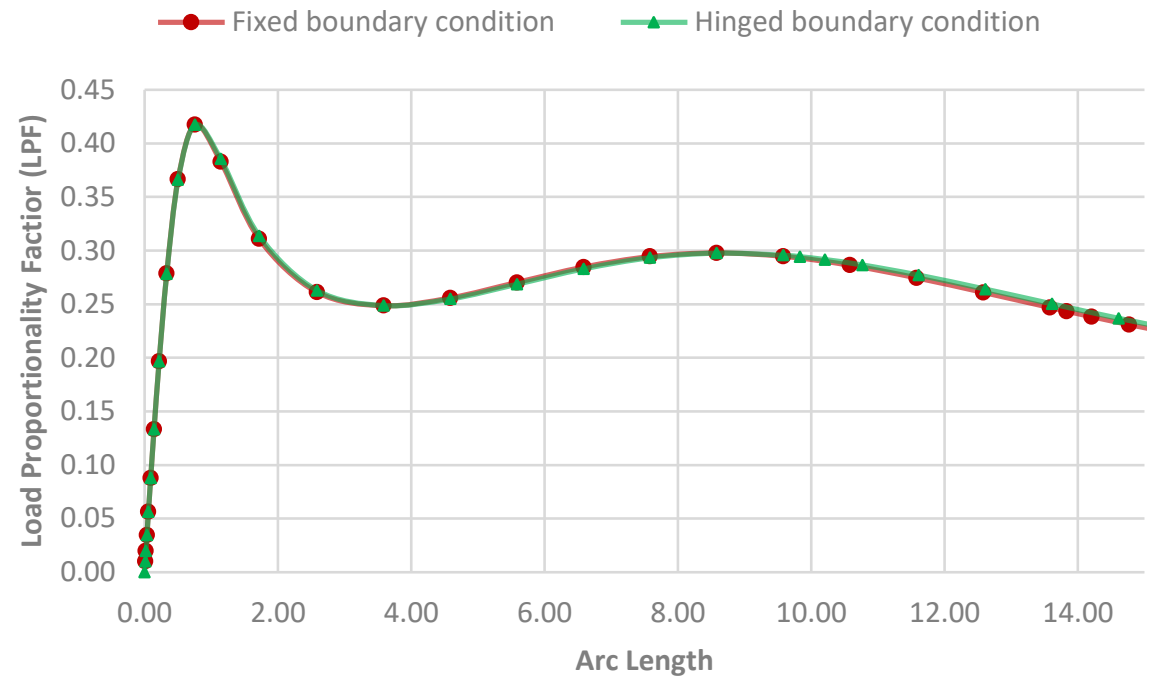

Figure A. 5 Comparison of boundary conditions - Model 5

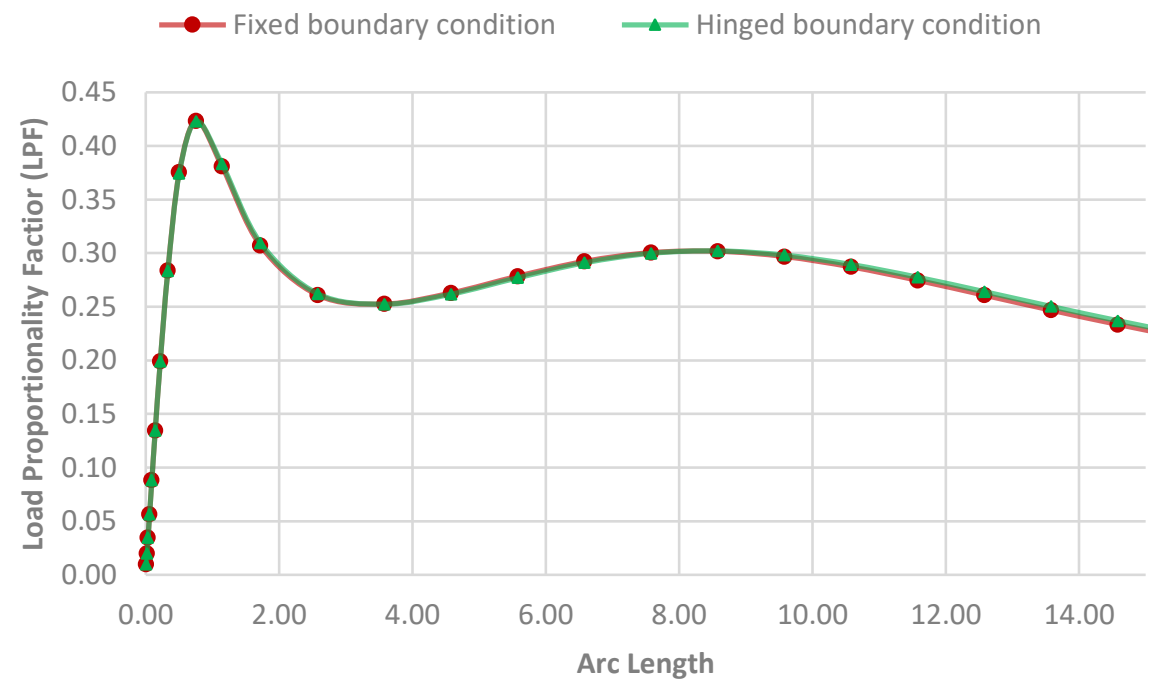

Figure A. 6 Comparison of boundary conditions - Model 6 


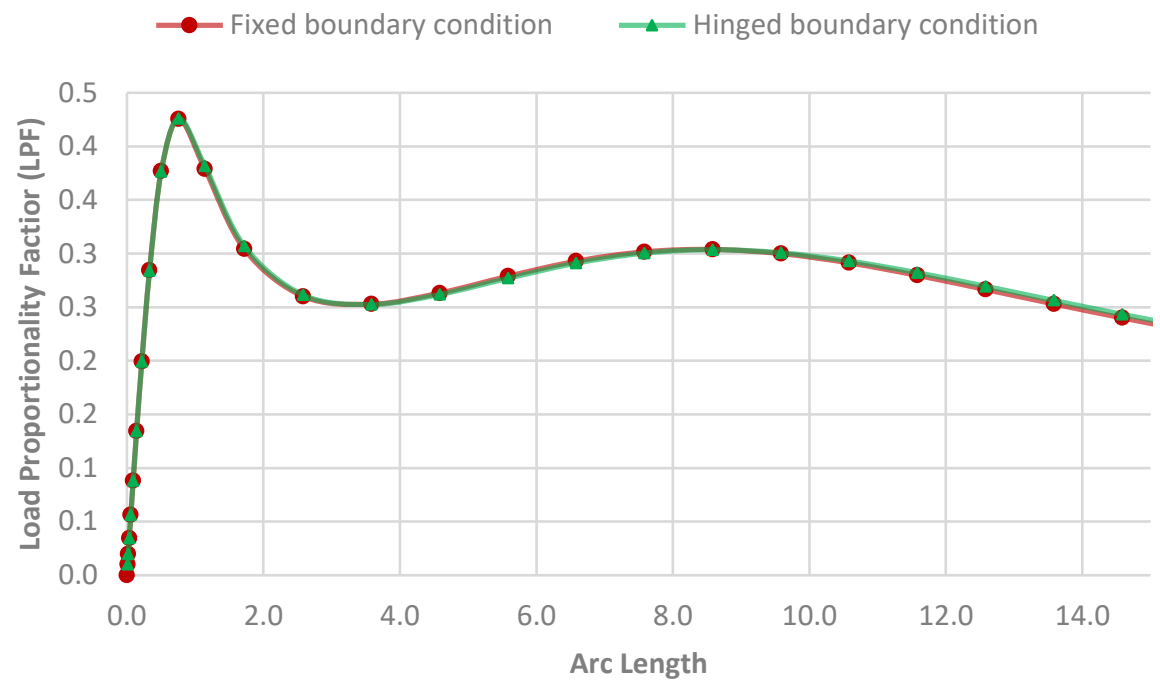

Figure A. 7 Comparison of boundary conditions - Model 7

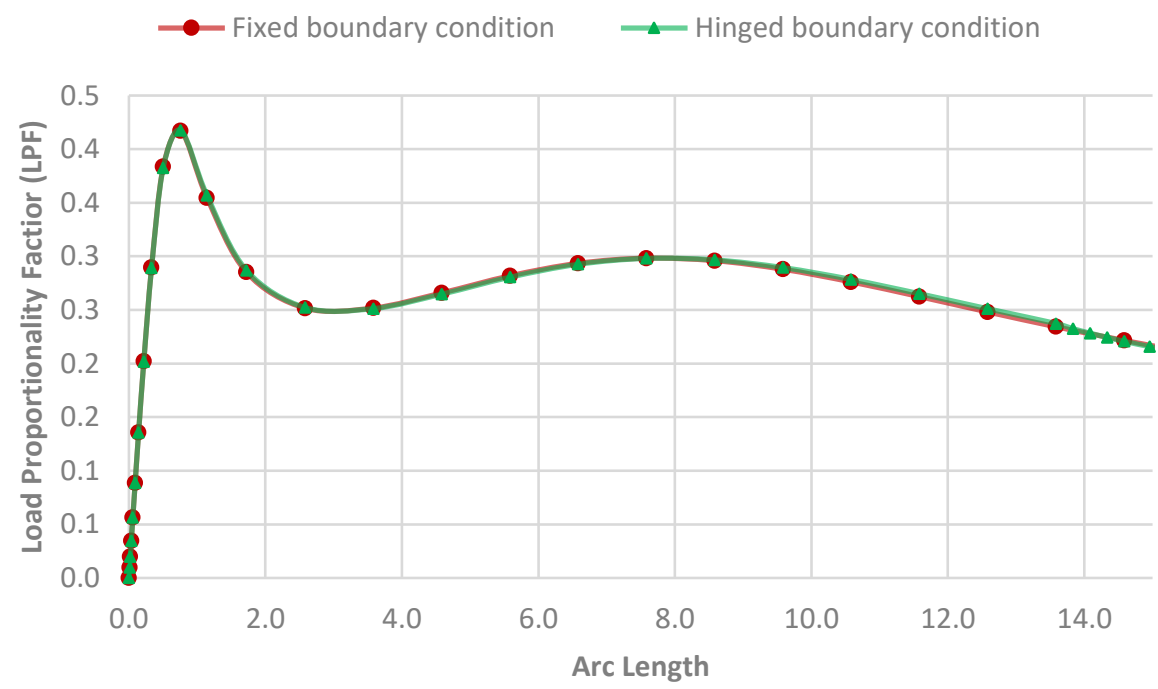

Figure A. 8 Comparison of boundary conditions - Model 8 


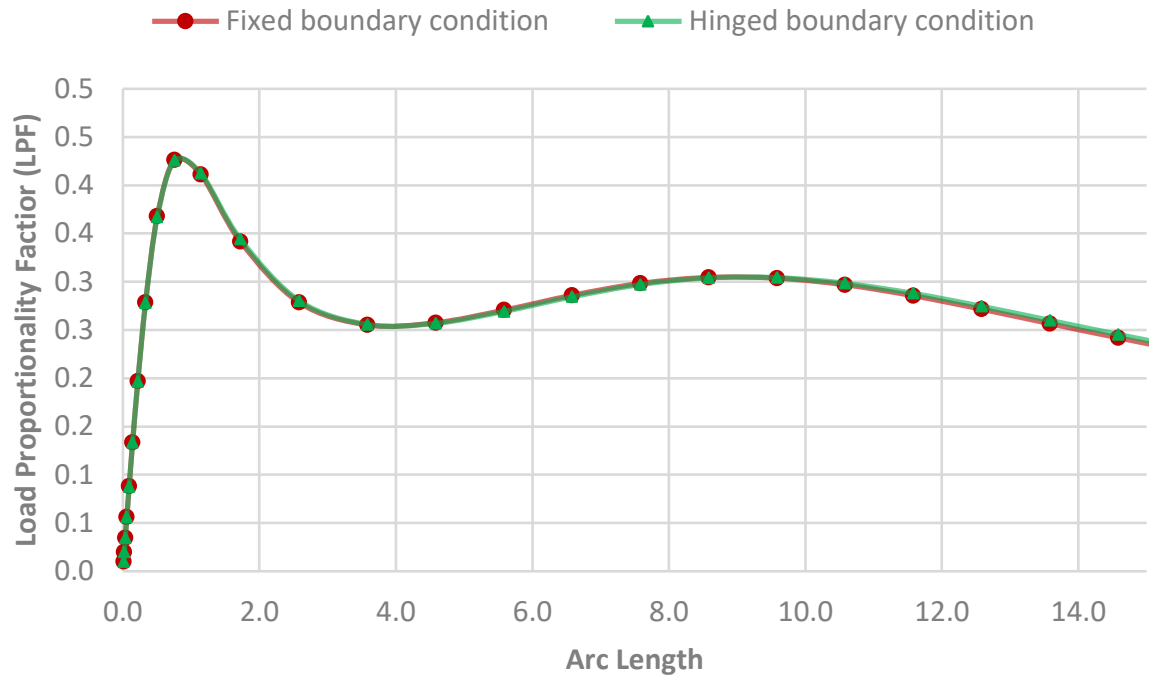

Figure A. 9 Comparison of boundary conditions - Model 9

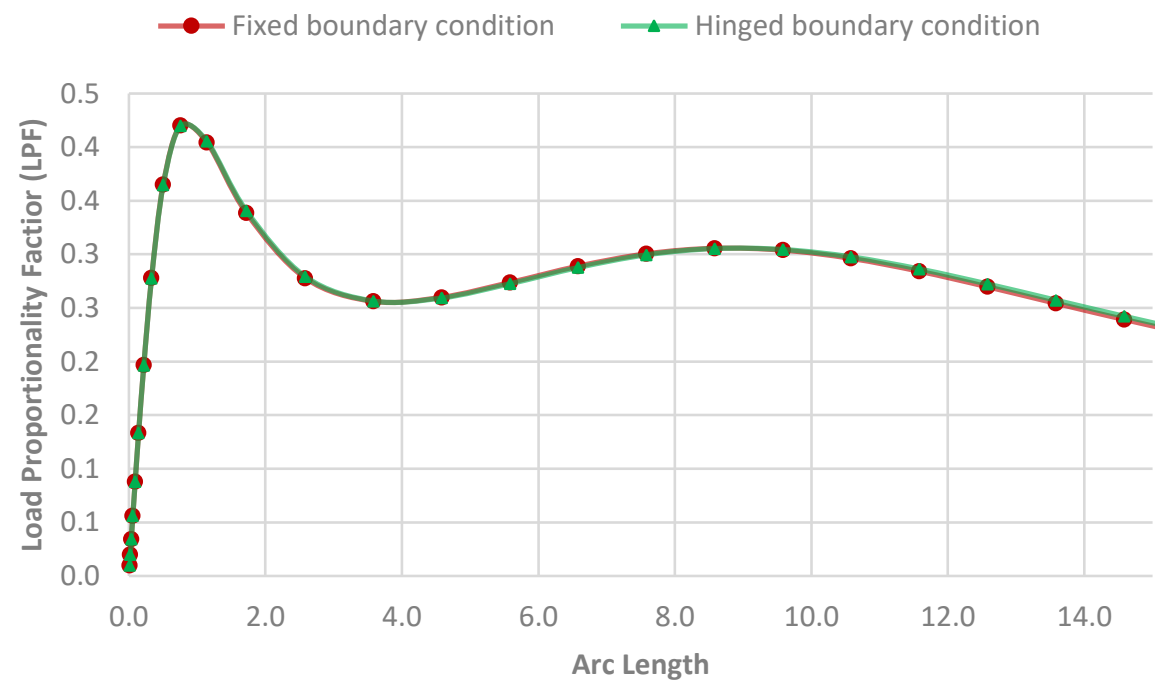

Figure A. 10 Comparison of boundary conditions - Model 10 


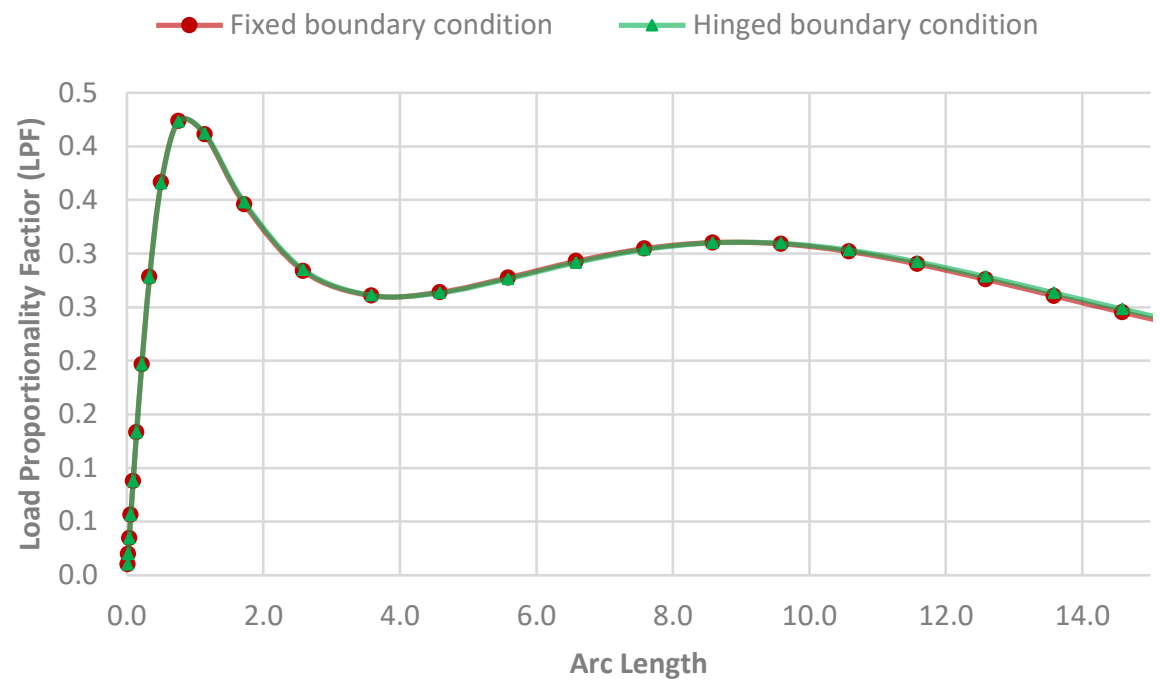

Figure A. 11 Comparison of boundary conditions - Model 11

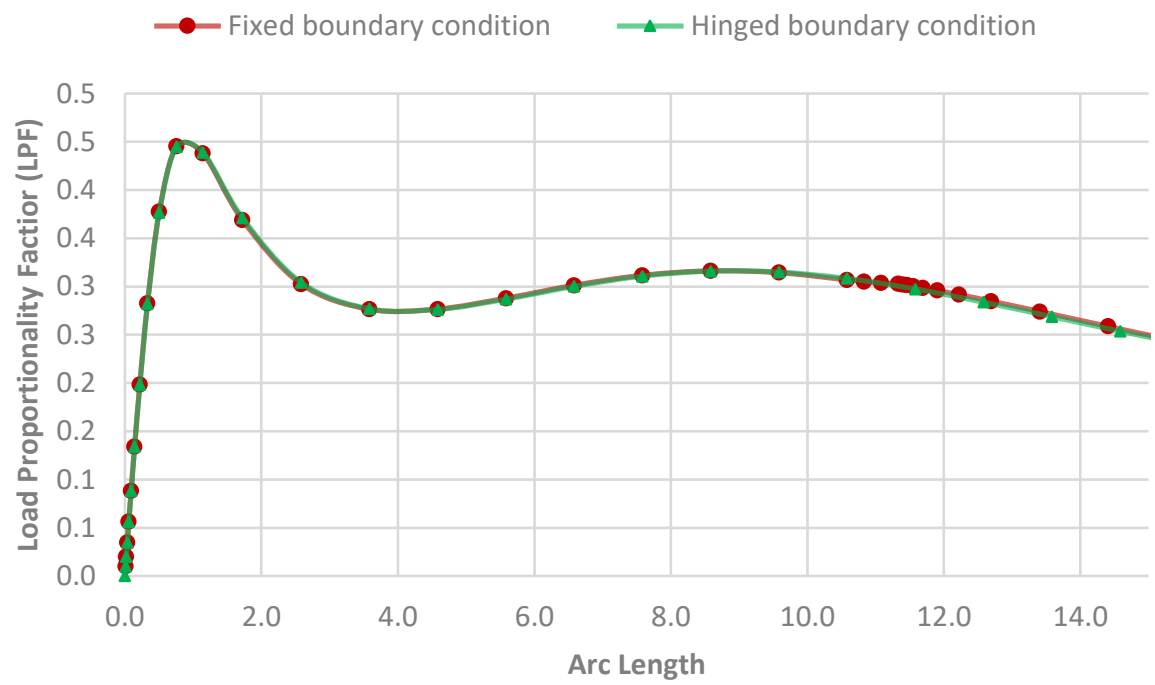

Figure A. 12 Comparison of boundary conditions - Model 12 


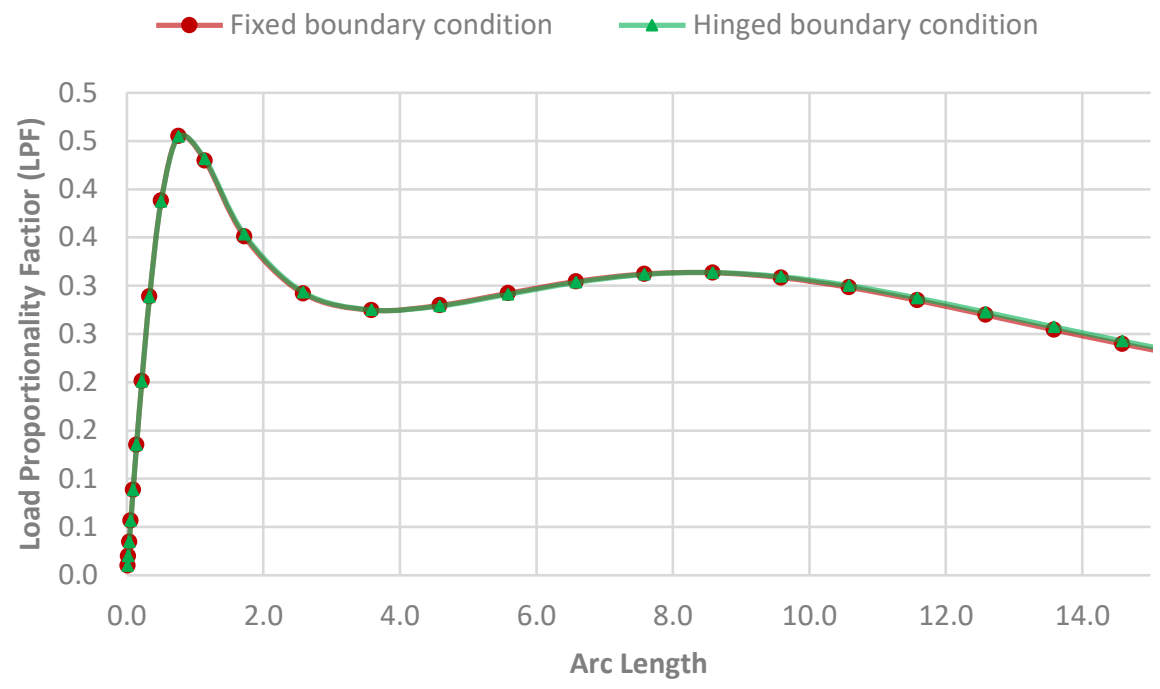

Figure A. 13 Comparison of boundary conditions - Model 13

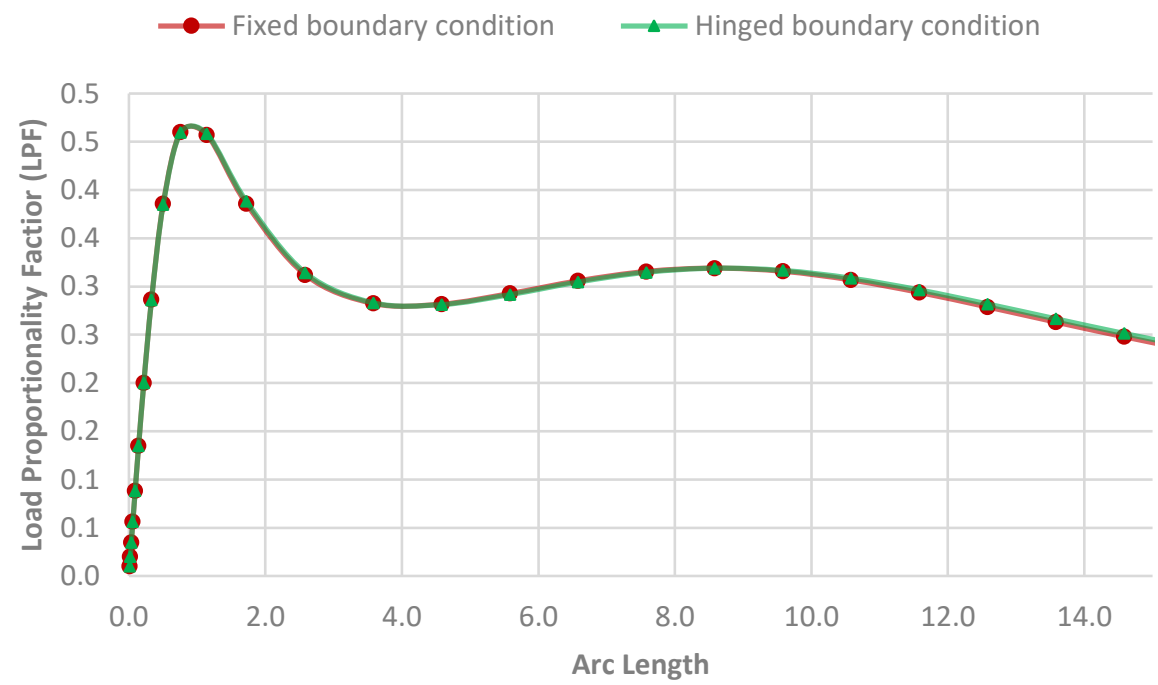

Figure A. 14 Comparison of boundary conditions - Model 14 


\section{APPENDIX B: INPUT FILE OF MODEL 2}

In this appendix, the ABAQUS input file for the seismic analysis of model 2 is presented. The mentioned parameters are defined as follows:

S4R: Four-node shell element with reduced integration

DL: Dead Load

Eq-H: Horizontal component of Earthquake

Eq-V: Vertical component of Earthquake

RebarOri-1: Cylindrical orientation of rebar

Section-1: Entire structure

Part-1-1.Base: Edge of the spherical shell

The input file considering the combined effect of the vertical and the horizontal component of earthquake for model 2 is given below:

** Job name: DL Model name: Model-2

*Preprint, echo=NO,

** Generated by: Abaqus/CAE 2016

*Preprint, echo $=\mathrm{NO}$, model $=\mathrm{NO}$, history $=\mathrm{NO}$, contact $=\mathrm{NO}$

**

** PARTS

$\star \star$

*Part, name=Part-1

** Section: Section-1

*Shell Section, elset=_I1, material=Concrete

$0.076,5$ 
${ }^{*}$ Rebar Layer, geometry=angular, orientation=RebarOri-1

Meridional, 0.000129, 2.43, 0., Rebar, 0., 1

*Rebar Layer, geometry=constant, orientation=RebarOri-1

Circumferential, 0.000129, 0.65, 0., Rebar, 90., 1

${ }^{* *}$ Region: (Section-1:Picked), (Controls:EC-1)

*Elset, elset=_12, internal

** Section: Section-1

*Shell Section, elset=_12, material=Concrete, controls $=E C-1$

$0.076,5$

*Rebar Layer, geometry=angular, orientation=RebarOri-1

Meridional, 0.000129, 2.43, 0., Rebar, 0., 1

*Rebar Layer, geometry=constant, orientation=RebarOri-1

Circumferential, 0.000129, 0.65, 0., Rebar, 90., 1

${ }^{*}$ End Part

**

${ }^{* *}$ ASSEMBLY

**

*Assembly, name=Assembly

**

*Instance, name=Part-1-1, part=Part-1

${ }^{\star}$ End Instance

**

*Nset, nset=Set-1, instance=Part-1-1, generate 
$1,3097, \quad 1$

*Elset, elset $=$ Set -1 , instance $=$ Part-1 1 , generate

*Surface, type=ELEMENT, name=Surf-1

_Surf-1_SPOS, SPOS

*End Assembly

**

** ELEMENT CONTROLS

$\star *$

*Section Controls, name $=E C-1$, second order accuracy $=$ YES

1., 1., 1 .

**

${ }^{* *}$ MATERIALS

**

*Material, name=Concrete

*Density

2.4,

*Elastic

$2.5029 e+07,0.17$

*Material, name=Rebar

*Density

7.85 ,

*Elastic

$2 \mathrm{e}+08,0.3$ 
${ }^{* *}$ BOUNDARY CONDITIONS

**

${ }^{* *}$ Name: BC-1 Type: Displacement/Rotation

*Boundary

Part-1-1.Base, 1, 1

** -

**

** STEP: Step-1

**

*Step, name=Step-1, nlgeom=YES

*Static

1., 1., 1e-05, 1.

**

** LOADS

**

** Name: DL Type: Body force

*Dload

Set-1, BY, -24.15

**

** OUTPUT REQUESTS

**

${ }^{*}$ Restart, write, frequency $=0$ 
${ }^{*}$ Output, field, frequency $=0$

${ }^{*}$ Output, history, frequency $=0$

${ }^{*}$ End Step

**

**

** STEP: Step-2

**

*Step, name=Step-2, nlgeom=YES, inc=150

*Static, riks

$0.01,1 ., 1 \mathrm{e}-15,1 .$, ,

**

** LOADS

**

${ }^{* *}$ Name: Eq-H Type: Surface traction

*Dsload, op $=\mathrm{NEW}$, follower $=\mathrm{NO}$, constant resultant $=\mathrm{YES}$

Surf-1, TRVEC, 1.5, 1., 0., 0.

** Name: Eq-V Type: Surface traction

*Dsload, op=NEW, follower=NO, constant resultant $=$ YES

Surf-1, TRVEC, 1., 0., -1., 0.

**

** OUTPUT REQUESTS

**

${ }^{*}$ Restart, write, frequency $=0$ 
** FIELD OUTPUT: F-Output-1, F-Output-2

**

${ }^{*}$ Output, field, variable=PRESELECT

**

** HISTORY OUTPUT: H-Output-1, H-Output-2

$\star \star$

${ }^{*}$ Output, history, variable=PRESELECT

${ }^{*}$ End Step 


\section{REFERENCES}

ACI Committee 318 (2008). Building Code Requirements for Structural Concrete, (ACI 318-08), American Concrete Institute. Farmington Hills, Mich.

ACI Committee 372 (2013). Guide to Design and Construction of Circular Wire- and strandWrapped Prestressed Concrete Structures, (ACI 372R-13), American Concrete Institute. Farmington Hills, Mich.

American Society of Civil Engineers (ASCE), Structural Engineering Institute (2010). Minimum design loads for buildings and other structures, ASCE/SEI 7-10. Reston, VA

Billington, D. P. (1965). Thin shell concrete structures. McGraw-Hill, New York.

Bushnell D. (1981). Computerized buckling analysis of shells. Air force wright aeronautical laboratories

Bushnell, D. (1967). Symmetric and Nonsymmetric Buckling of Finitely Deformed Eccentrically Stiffened Shells of Revolution. AIAA Journal, Vol. 5, No. 8, pp. 1435-1462

Carlson, R. L., Sendelbeck R. L. and Hoff N. J. (1967). Experimental Studies of Buckling of Complete spherical Shells. Experimental Mechanics, Vol. 7, No. 7, pp. 281-288

Danielson, D. A. (1974). Theory of Shell Stability. Edited by Fung Y. C. and Sechler E.E. Thin-Shell Structures. Theory, experiment and design. Prentice-Hall, Inc. Englewood Cliffs, New Jersey.

Donnell, L. H. (1934). A new Theory for the Buckling of Thin Cylinders under Axial Compression and Bending. Transactions of the American Society of Mechanical Engineers, vol. 56 , pp. $795-806$

Farnsworth, Jr. D. B. (1998) Behavior of Shell Structures. Massachusetts Institute of 
Technology

Gupta, P.K., Gupta, N.K. (2008). "A study of axial compression of metallic hemispherical domes”. Journal of materials processing technology Elsevier, Vol.209, pp. 2175-2179

Hamed, E, Bradford, M and Gilbert, I. (2010). Nonlinear long-term behaviour of spherical shallow thin-walled concrete shells of revolution. International Journal of Solids and Structures Elsevier, Vol. 47, No. 2, pp. 204-215

Hamed, E, Bradford, M, Gilbert, I., and Chang, Z. (2011). Analytical model and experimental study of failure behavior of thin-walled shallow concrete domes. Journal of Structural Engineering ASCE, Vol. 137, No. 1, pp. 88-99

Heger, F. J. and Zarghamee, M. S. (1983). Buckling of Thin Concrete Domes. ACI Journal, Vol. 80, No. 45, pp. 487-500

Hooke, R. (1678). De Potentia Restitutiva, or of Spring. Explaining the Power of Springing Bodies. London.

Huang, N. (1963). Unsymmetrical Buckling of Thin Shallow Spherical Shells (Technical Report No. 15). Cambridge, Massachusetts: Division of Engineering and Applied Physics, Harvard University.

Karman, T. V. and Tsien, H. (1939). The Buckling of Spherical Shells by external pressure. Journal of the Aeronautical Sciences, Vol 17 pp. 43-50

Kirchhoff, G. (1859). Über das Gleichgewicht und die Bewegung einer elastischen Scheibe. Journal für reine und angewandte Mathematik, Vol. 56, pp.285-313.

Kraus, H (1967). Thin elastic shells. State University of New York.

Krenzke, M. A. and Kiernan, T. J. (1965). The Effect of Initial Imperfections on the Collapse Strength of Deep Spherical Shells (Technical Report no. 1757). Department of the Navy, 
Washington, D.C.

Love, A. E. H. (1888). The small Free Vibrations and Deformation of a Thin Elastic Shell. Philosophical Transactions of the Royal Society of London. A, Vol. 179, pp. 491-546

Mekjavić, I. (2011). Buckling Analysis of Concrete Spherical Shells. Tehnički vjesnik, Vol. 18, pp. 633-639Sechler, E. E. and Bollay, W. (1939) Some Investigations of the General Instability of Stiffened Metal Cylinders, California Institute of Technology

Timoshenko, S.P. (1936). Theory of Elastic Stability. $1^{\text {st }}$ Edition, McGraw-Hill Book Company, Inc., New York.

Timoshenko, S.P. and Gere, J.M. (1961). Theory of Elastic Stability. 2nd Edition, McGrawHill Book Company, Inc., New York.

Poisson, S.D. (1827). Note sur l'Extension des Fils et des Plaques élastiques. Annls Chim, Phys. Vol 36. pp. 384-387

Smith, M. (2016). ABAQUS/Standard User's Manual, Version 2016. Providence, RI: Simulia.

Vandepitte, D, Rathe, D. and Weyneis, G. (1980). An experimental investigation into the buckling and creep buckling of shallow spherical caps subjected to uniform radial pressure. IASS World Congress on Shell and Spatial Structures, Vol. 1, pp. 1-15

Ventsel, E. and Krauthammer, T. (2001). Thin Plates and Shells. Boca Raton: CRC Press. Zolqadr, E (2017). Buckling of spherical concrete shells. Ryerson University 